\title{
DUST AND THE TYPE II-PLATEAU SUPERNOVA 2004dj
}

\author{
W. P. S. Meikle ${ }^{1}$, R. Kotak ${ }^{2}$, D. Farrah ${ }^{3}$, S. Mattila ${ }^{4,5}$, S. D. Van DyK ${ }^{6}$, A. C. Andersen ${ }^{7}$, R. Fesen $^{8}$, \\ A. V. FilipPenKo ${ }^{9}$, R. J. Foley ${ }^{9,10,14}$, C. Fransson ${ }^{5}$, C. L. Gerardy ${ }^{11}$, P. A. HöFlich ${ }^{11}$, P. LundQVist ${ }^{5}$, \\ M. Pozzo ${ }^{12}$, J. Sollerman ${ }^{5,7}$, and J. C. WheEler ${ }^{13}$ \\ ${ }^{1}$ Astrophysics Group, Blackett Laboratory, Imperial College London, Prince Consort Road, London SW7 2AZ, UK; p.meikle@imperial.ac.uk \\ 2 Astrophysics Research Centre, School of Mathematics and Physics, Queen's University Belfast, BT7 1NN, UK \\ ${ }^{3}$ Astronomy Centre, Department of Physics and Astronomy, University of Sussex, Brighton BN1 9QJ, UK \\ ${ }^{4}$ Tuorla Observatory, Department of Physics and Astronomy, University of Turku, Väisäläntie 20, FI-21500 Piikkiö, Finland \\ ${ }^{5}$ Department of Astronomy, Stockholm University, AlbaNova, SE-10691 Stockholm, Sweden \\ ${ }^{6}$ Spitzer Science Center, 220-6 Caltech, Pasadena, CA 91125, USA \\ ${ }^{7}$ Dark Cosmology Centre, Niels Bohr Institute, University of Copenhagen, Juliane Maries Vej 30, 2100 Copenhagen $\varnothing$, Denmark \\ ${ }^{8}$ Department of Physics and Astronomy, 6127 Wilder Lab., Dartmouth College, Hanover, NH 03755, USA \\ ${ }^{9}$ Department of Astronomy, University of California, Berkeley, CA 94720-3411, USA \\ ${ }^{10}$ Harvard-Smithsonian Center for Astrophysics, 60 Garden Street, Cambridge, MA 02138, USA \\ ${ }^{11}$ Department of Physics, Florida State University, 315 Keen Building, Tallahassee, FL 32306-4350, USA \\ 12 Department of Earth Sciences, University College London, London WC1E 6BT, UK \\ ${ }^{13}$ Astronomy Department, University of Texas, Austin, TX 78712, USA \\ Received 2010 November 30; accepted 2011 March 10; published 2011 April 25
}

\begin{abstract}
We present mid-infrared (MIR) spectroscopy of a Type II-plateau supernova, SN 2004dj, obtained with the Spitzer Space Telescope, spanning 106-1393 days after explosion. MIR photometry plus optical/near-IR observations are also reported. An early-time MIR excess is attributed to emission from non-silicate dust formed within a cool dense shell (CDS). Most of the CDS dust condensed between 50 days and 165 days, reaching a mass of $0.3 \times 10^{-5} M_{\odot}$. Throughout the observations, much of the longer wavelength $(>10 \mu \mathrm{m})$ part of the continuum is explained as an IR echo from interstellar dust. The MIR excess strengthened at later times. We show that this was due to thermal emission from warm, non-silicate dust formed in the ejecta. Using optical/near-IR line profiles and the MIR continua, we show that the dust was distributed as a disk whose radius appeared to be shrinking slowly. The disk radius may correspond to a grain destruction zone caused by a reverse shock which also heated the dust. The dust-disk lay nearly face-on, had high opacities in the optical/near-IR regions, but remained optically thin in the MIR over much of the period studied. Assuming a uniform dust density, the ejecta dust mass by 996 days was $(0.5 \pm 0.1) \times 10^{-4} M_{\odot}$ and exceeded $10^{-4} M_{\odot}$ by 1393 days. For a dust density rising toward the center the limit is higher. Nevertheless, this study suggests that the amount of freshly synthesized dust in the SN 2004dj ejecta is consistent with that found from previous studies and adds further weight to the claim that such events could not have been major contributors to the cosmic dust budget.
\end{abstract}

Key words: circumstellar matter - dust, extinction - supernovae: general - supernovae: individual (SN 2004dj)

Online-only material: color figures

\section{INTRODUCTION}

Massive stars explode via core collapse and ejection of their surrounding layers (e.g., Arnett et al. 1989, and references therein). The extent to which core-collapse supernovae (CCSNe) are, or have been, a major source of dust in the universe is of great interest. Of particular concern is the evidence of enormous amounts of dust $\left(\gtrsim 10^{8} M_{\odot}\right)$ in galaxies at high redshifts $(z \gtrsim 5)$. This comes from a variety of observations such as submillimeter and near-infrared (NIR) studies of the most distant quasars (Bertoldi et al. 2003; Maiolino et al. 2004), obscuration by dust of quasars in damped Ly $\alpha$ systems (Pei et al. 1991), and measurements of metal abundances in these systems (Pettini et al. 1997). Until recently, the scenario of dust from asymptotic giant branch (AGB) stars tended to be rejected since it was thought that their progenitors would not yet have evolved off the main sequence. However, Valiante et al. (2009) and Dwek \& Cherchneff (2011) have argued that, under certain circumstances, AGB stars may make some contribution to the dust budget at high redshifts. Both studies nevertheless cannot

\footnotetext{
${ }^{14}$ Clay Fellow.
}

rule out a supernova $(\mathrm{SN})$ contribution. In this paper, we examine the SN option through observations of a nearby core-collapse event.

CCSNe arising from short-lived Population III stars might seem to be a viable alternative. It is estimated that each SN must produce $0.1-1 M_{\odot}$ of dust to account for the highredshift observations (Dwek et al. 2007; Meikle et al. 2007). Such masses have been predicted in models of dust formation in CCSNe (Todini \& Ferrara 2001; Nozawa et al. 2003), although more recent calculations by Cherchneff \& Dwek (2010) revise such estimates downward by a factor of $\sim 5$. Perhaps even more problematic is the fact that actual dustmass measurements in $\mathrm{CCSNe}$ and $\mathrm{SN}$ remnants yield values not exceeding, respectively, $10^{-3} M_{\odot}$ and $10^{-2} M_{\odot}$, although only a handful of such measurements exist. (For a summary of this topic see, for example, Kotak et al. 2009, Section 1.) The Spitzer Space Telescope (hereafter Spitzer; Werner et al. 2004) provided an excellent opportunity for us to test the ubiquity of dust condensation in a larger number of CCSNe.

Newly condensed dust in CCSNe can be detected by its attenuating effects on optical/NIR light and/or via thermal emission from the grains in the ejecta. Prior to Spitzer, the 
only evidence of dust condensation in typical CCSNe was in the Type II-plateau (IIP) SN 1988H (Turatto et al. 1993) and SN 1999em (Elmhamdi et al. 2003). However, the light curve data used to type SN $1988 \mathrm{H}$ were sparse. In the case of SN 1999em, Elmhamdi et al. used optical line suppression to infer a dust mass lower limit of about $10^{-4} M_{\odot}$. With the launch of Spitzer, we were at last provided with a facility for highsensitivity spectroscopy and imaging of nearby CCSNe over the mid-infrared (MIR) range, covering the likely peak of the dust thermal emission spectrum. This can provide a superior measure of the total flux, temperature, and possibly dust emissivity than can be achieved at shorter wavelengths. Moreover, the longerwavelength coverage of Spitzer allows us to detect cooler grains and see more deeply into dust clumps than was previously possible for typical nearby CCSNe. In this paper, we present our late-time Spitzer observations of the Type IIP SN 2004dj. We use these observations to study the dust production in this SN.

The paper is arranged as follows. In Section 1.1, we summarize and discuss previous observations of SN 2004dj. In Section 2, we present MIR (Spitzer) photometric and spectroscopic observations of SN 2004dj, extending to more than three years after the explosion. This MIR coverage is one of the most extensive ever achieved for an SN IIP. We also present latetime optical and NIR photometry and spectra of SN 2004dj. In Section 3, we analyze these data. Corrections are derived in Section 3.1 for the effects of the line-of-sight cluster S96, and in Section 3.2 the mass of ejected ${ }^{56} \mathrm{Ni}$ is determined. We compare the data with blackbodies in Section 3.3 in order to assess the likely number and nature of the contributing sources. In Section 3.4, the origins of the IR radiation are examined in detail. The work is then summarized in Section 4.

\subsection{SN $2004 d j$}

SN 2004dj was discovered in the nearby spiral galaxy NGC 2403 on 2004 July 31 by Nakano et al. (2004) and was classified as a normal Type IIP SN by Patat et al. (2004). It was the nearest such event in over three decades; the host galaxy lies within the M81 group. In Kotak et al. (2005), we adopted a distance to NGC 2403 (and the SN) of $3.13 \pm 0.15 \mathrm{Mpc}$ (statistical errors only), this being the Cepheid-derived, zerometallicity value reported by Freedman et al. (2001) using the Udalski et al. (1999) period-luminosity slopes. We continue to use this distance in the work presented here. Vinkó et al. (2006) have estimated the distance to SN 2004dj using a combination of the Freedman et al. value plus their own expanding photosphere method (EPM) and standard candle estimates. This yields an average distance of $3.47 \pm 0.29 \mathrm{Mpc}$, implying that the SN luminosity could be $\sim 20 \%$ larger than the values used herein.

The progenitor of SN 2004dj was almost certainly a member of the compact star cluster Sandage 96 (S96) (Sandage 1984; Bond et al. 2004; Filippenko et al. 2004; Maíz-Apellániz et al. 2004; Wang et al. 2005; Vinkó et al. 2006). The cluster age is variously estimated to be $14 \pm 2$ Myr (Maíz-Apellániz et al. 2004), 20 Myr (Wang et al. 2005), and 10-16 Myr (Vinkó et al. 2009). The main-sequence mass of the progenitor is estimated at $\sim 15 M_{\odot}$ (Maíz-Apellániz et al. 2004; Kotak et al. 2005), $\sim 12 M_{\odot}$ (Wang et al. 2005), and 12-20 $M_{\odot}$ (Vinkó et al. 2009). Maíz-Apellániz et al. (2004) and Kotak et al. (2005) favor a red supergiant (RSG) progenitor.

Guenther \& Klose (2004) used echelle spectroscopy of $\mathrm{Na}$ I D absorption lines in NGC 2403 along the line of sight to $\mathrm{SN} 2004 \mathrm{dj}$ to infer a heliocentric velocity of $+164.8 \pm$ $0.1 \mathrm{~km} \mathrm{~s}^{-1}$. This is somewhat larger than the heliocentric velocity of the nuclear region of NGC 2403 of $\sim 130 \mathrm{~km} \mathrm{~s}^{-1}$ given in the SIMBAD and NED databases, but this is not surprising given the likely dispersion of velocities within the host galaxy. Indeed, Vinkó et al. (2006) point out that H I mapping of NGC 2403 (Fraternali et al. 2001) suggests that the true radial velocity of the SN 2004dj barycenter is about $+221 \mathrm{~km} \mathrm{~s}^{-1}$. We adopt this value here.

There is no firm consensus about the value of the reddening to SN 2004dj. Stellar population fitting for S96 yields total (Galactic + host) $E(B-V)$ values of $0.17 \pm 0.02 \mathrm{mag}$ (Maíz-Apellániz et al. 2004), $0.35 \pm 0.05 \mathrm{mag}$ (Wang et al. 2005), and $0.1 \pm 0.05 \mathrm{mag}$ (Vinkó et al. 2009). Direct color comparisons of SN 2004dj with other CCSNe yield $E(B-V)$ values of $\sim 0 \mathrm{mag}$ (Zhang et al. 2006), $0.06 \mathrm{mag}$ (Chugai 2006), and $0.07 \pm 0.1 \mathrm{mag}$ (Vinkó et al. 2006). Perhaps most significantly, the resolved Na I D observations of Guenther \& Klose (2004) yield a host-only $E(B-V)$ value of just $0.026 \pm$ $0.002 \mathrm{mag}$. The smaller values of $E(B-V)$ obtained using direct measurements toward SN 2004dj suggest that the SN actually lies near the front of S96. Based on the extinction maps of Schlegel et al. (1998), Chugai et al. (2005) find a Galactic reddening of $E(B-V)=0.062$ mag while Zhang et al. (2006) report $E(B-V)=0.04 \mathrm{mag}$ (the same value as obtainable from NED). We therefore adopt a Galactic $E(B-V)=0.05 \pm$ $0.01 \mathrm{mag}$. If we add the Guenther \& Klose (2004) host value to the Galactic value, we obtain $E(B-V)=0.076 \pm 0.01 \mathrm{mag}$, or $A_{V}=0.24 \pm 0.03$ mag for a Cardelli et al. (1989) extinction law with $R_{V}=3.1$. Given the range of published values, in the present work we used a total extinction $A_{V}=0.31 \mathrm{mag}$, the same as that preferred by Vinkó et al. (2009). Adoption of even the largest published value of $E(B-V)$ would increase our shortest MIR wavelength $(3.6 \mu \mathrm{m})$ flux by just a few percent.

Estimates of the explosion date of SN 2004dj vary by several weeks. On the basis of an early-time spectrum, Patat et al. (2004) placed the explosion at about 2004 July 14. This is consistent with the date obtained by Beswick et al. (2005), who used the radio $L_{\text {peak }}$ versus rise time relation (Weiler et al. 2002) to yield an explosion date between 2004 July 11 and 31. On the other hand, based on the EPM method, Vinkó et al. (2006) derive an explosion date as early as 2004 June 30 . On the assumptions that the light curve of SN 1999gi was typical of SNe IIP and that the SN 2004dj plateau was of similar length, Chugai et al. (2005) obtained an even earlier explosion date: 2004 June 13. Likewise, assuming similar evolution between SN 2004dj and SN 1999em, Zhang et al. (2006) find an explosion date of 2004 June 11. Nevertheless, partly as a compromise with the later Patat et al. (2004) value, Chugai (2006) subsequently adopted 2004 June 28 as the date of the explosion. Chugai et al. (2007) also used this explosion date. Given the weight of evidence for a later explosion date, we reject that preferred by Zhang et al. (2006). In Kotak et al. (2005), we adopted an explosion date of 2004 July 10, or MJD = 53196.0. We use the same explosion date here but recognize that there is an uncertainty of about \pm 7 days. All epochs will be with respect to MJD $=53196.0$ (i.e., $t=0$ days).

Optical light curves of SN 2004dj are presented by Korcáková et al. (2005), Chugai et al. (2005), Leonard et al. (2006), Vinkó et al. (2006, 2009), and Zhang et al. (2006). The VR light curves fell by $10 \%$ and $90 \%$ of the total decline from the plateau to the start of the radioactive tail at, respectively, epochs $70 \pm 5$ days and $96 \pm 3$ days. At the start of the radioactive tail, the SN luminosity was about $15 \%$ of the plateau value. During the early nebular phase (up to $\sim 300$ days) the $V$ band declined 


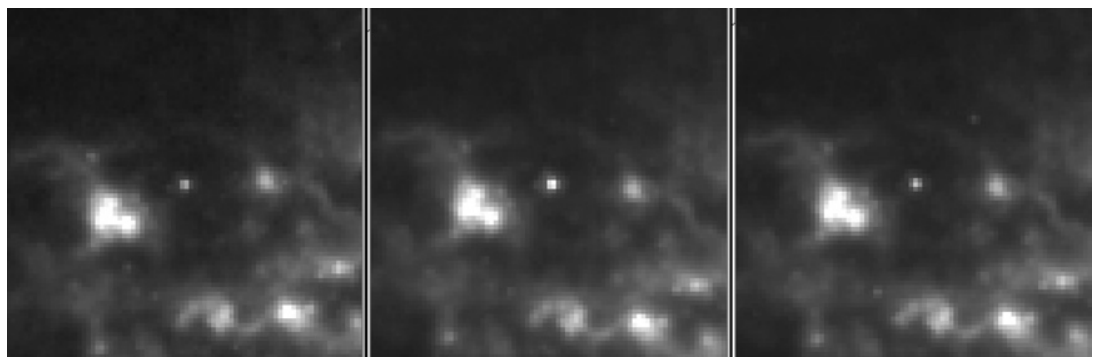

Figure 1. Sequence of images at $8 \mu \mathrm{m}$ at 257 days (LHS), 621 days (middle), and 996 days (RHS). SN 2004dj is the point source at the center of each image. The supernova is clearly brighter on 621 days. This is due to the epoch being close to the peak of the thermal emission from the ejecta dust at this wavelength (see Figure 2 ). The fields are about 2 arcmin across $\left(\sim 1.8 \mathrm{kpc}\right.$ at the distance of the supernova). North is $\sim 30^{\circ}$ clockwise from the upward vertical.

at about 1.1 mag (100 days) $)^{-1}$ (Vinkó et al. 2006), which is typical for an SN IIP. On the basis of the light curves, the mass of ejected ${ }^{56} \mathrm{Ni}$ has been estimated at $0.02-0.03 M_{\odot}$ (Chugai et al. 2005; Kotak et al. 2005; Vinkó et al. 2006; Zhang et al. 2006). Notwithstanding, in the present work we argue that these authors have overestimated the ${ }^{56} \mathrm{Ni}$ mass and that the true mass is more like $0.01 M_{\odot}$.

At the end of the plateau phase, SN 2004dj exhibited a remarkable and rapid change in some of its prominent optical lines, especially $\mathrm{H} \alpha$. At 89 days the $\mathrm{H} \alpha$ profile still had a typical P Cygni morphology with a symmetric peak blueshifted by only about $-270 \mathrm{~km} \mathrm{~s}^{-1}$ after correction for the heliocentric velocity of the SN. Yet, by just $\sim 10$ days later the profile had developed a strong asymmetric profile with a peak at $-1610 \mathrm{~km} \mathrm{~s}^{-1}$ (Chugai et al. 2005). As time went by during the first year, this blueshift gradually decreased and the asymmetry became less pronounced. Chugai (2006) interpreted this unusual behavior as being due to the gradual emergence of an asymmetric, bipolar jet whose more massive component is traveling toward the observer. They propose that the lines are driven by the radioactive decay of spherical fragments of ${ }^{56} \mathrm{Co}$ cocooned in metals and helium, lying within the core. Using spectropolarimetry, Leonard et al. (2006) also found evidence for departure from spherical symmetry. The polarization was observed to increase dramatically at the end of the plateau phase, implying the presence of significant asphericity in the inner regions of the ejecta.

Early-time evidence of a significant circumstellar medium (CSM) around the progenitor of SN 2004dj has also been reported. Radio emission was detected by Stockdale et al. (2004) at 23 days, by Beswick et al. (2005) between 26 days and 145 days, and by Chandra \& Ray (2004) at 33 days and 43 days. The SN was also detected at X-ray wavelengths (Pooley \& Lewin 2004) at 30 days. The X-ray luminosity was about three times that of the Type IIP SN $1999 \mathrm{em}$ and nearly 15 times that of the Type IIP SN 1999gi at similar epochs. Beswick et al. (2005) point out that both types of emission arise from a shocked CSM. Chugai et al. (2007) have used the presence of a highvelocity absorption component in the $\mathrm{H} \alpha$ line during the late photospheric phase to deduce the presence of a cool dense shell (CDS), with a mass of $3.2 \times 10^{-4} M_{\odot}$, produced by interaction of the ejecta with the pre-existing CSM. To this evidence for a CDS, we add our observation of an early-time IR echo in SN 2004dj (see Section 3.4.2).

In Kotak et al. (2005), we presented MIR photometric and spectroscopic observations of SN 2004dj at epochs 97-137 days after explosion. Simultaneous modeling of the fundamental (1-0) and first overtone (2-0) of CO was carried out. The results favor a $15 M_{\odot}$ RSG progenitor and indicate post-explosion CO formation in the range $2000-4000 \mathrm{~km} \mathrm{~s}^{-1}$. Kotak et al. (2005) also noted an underlying NIR continuum. A possible origin in CSM dust was suggested, but RSGs in S96 were favored as the more likely cause. Nevertheless, in the present work (Section 3.2.2) we find that the bulk of the early-time NIR and MIR continuum is most plausibly explained as an IR echo from CDS dust. The presence of $1.7 \times 10^{-4} M_{\odot}$ of $\mathrm{Ni}^{+}$in the ejecta was also deduced by Kotak et al. (2005).

In summary, while the early-time optical light curves and spectra of SN 2004dj are typical of a Type IIP event, its early radio, MIR, and X-ray behavior point to an exceptionally strong ejecta/CSM interaction. Moreover, its earlier nebular-phase spectra imply an atypically asymmetric core. SN 2004dj is only "typical" in some respects.

\section{OBSERVATIONS}

\subsection{Mid-infrared Photometry}

Imaging at 3.6, 4.5, 5.8, and $8.0 \mu \mathrm{m}$ was obtained with the Infrared Array Camera (IRAC; Fazio et al. 2004), at 16 and $22 \mu \mathrm{m}$ with the Peak-up Array (PUI) of the Infrared Spectrograph (IRS; Houck et al. 2004), and at $24 \mu \mathrm{m}$ with the Multiband Imaging Photometer for Spitzer (MIPS; Rieke et al. 2004). Imaging observations spanned epochs 89.1-1393.3 days, plus four observations, at $3.6 \mu \mathrm{m}$ only, covering 1953.9-2143.5 days. Aperture photometry was performed on the images using the Starlink package GAIA (Draper et al. 2002). A circular aperture of radius $\sim 3$ ".7 was used for the photometry. The background flux was measured and subtracted by using a concentric sky annulus having inner and outer radii of 1.5 and 2.2 times the aperture radius, respectively. These parameters were chosen as a compromise between maximizing the sampled fraction of source flux and minimizing the effects of the bright, complex background. The aperture radius corresponds to $\sim 55 \mathrm{pc}$ at the distance of SN 2004dj. The aperture was centered according to the SN WCS coordinates. Aperture corrections were derived from the IRAC and MIPS point-response function frames available from the Spitzer Science Center and ranged from $\times 1.16$ at $3.6 \mu \mathrm{m}$ to $\times 2.79$ at $24 \mu \mathrm{m}$. A $2 \sigma$ clipped mean sky estimator was used, and the statistical error was estimated from the variance within the sky annuli. Fluxing errors due to uncertainties in the aperture corrections are about $\pm 5 \%$.

The MIR photometry is presented in Table 1. The Spitzer programs from which the imaging data were taken are listed in the caption. The pre-explosion MIR flux of S96 has not been measured, so the tabulated values are uncorrected for $\mathrm{S} 96$. An estimate of the S96 contribution is given at the bottom of the table. The effect of emission from S96 is discussed in Section 3.1. A temporally varying point source at the $\mathrm{SN}$ position is clearly visible in all bands. Figure 1 shows a sequence of images at $8.0 \mu \mathrm{m}$ at 257,621 , and 996 days. The SN is clearly 
Table 1

Mid-IR Photometry of SN 2004dj

\begin{tabular}{|c|c|c|c|c|c|c|c|c|c|c|}
\hline \multirow[t]{3}{*}{ Date } & \multirow[t]{3}{*}{ MJD } & \multirow{3}{*}{$\begin{array}{c}\text { Epoch }^{\mathrm{b}} \\
\text { (days) }\end{array}$} & \multirow{3}{*}{$\begin{array}{l}t_{\exp } \\
\text { (s) }\end{array}$} & \multicolumn{7}{|c|}{ Flux $(\mathrm{mJy})^{\mathrm{a}}$} \\
\hline & & & & \multicolumn{4}{|c|}{ IRAC } & \multicolumn{2}{|c|}{ PUI } & \multirow{2}{*}{$\begin{array}{l}\text { MIPS } \\
24 \mu \mathrm{m}\end{array}$} \\
\hline & & & & $3.6 \mu \mathrm{m}$ & $4.5 \mu \mathrm{m}$ & $5.8 \mu \mathrm{m}$ & $8.0 \mu \mathrm{m}$ & $16 \mu \mathrm{m}$ & $22 \mu \mathrm{m}$ & \\
\hline 2004 Oct $7^{c}$ & 53285.1 & 89.1 & 134 & $11.25(2)$ & $10.00(2)$ & $6.85(3)$ & $4.32(4)$ & $\ldots$ & $\ldots$ & $\ldots$ \\
\hline 2004 Oct $8^{\mathrm{d}}$ & 53286.4 & 90.4 & 107 & $10.94(2)$ & $10.01(2)$ & $6.74(3)$ & $4.09(5)$ & $\ldots$ & $\ldots$ & $\ldots$ \\
\hline 2004 Oct $12^{\mathrm{d}}$ & 53290.1 & 94.1 & 107 & $8.30(2)$ & $9.71(2)$ & $6.02(3)$ & $3.45(4)$ & $\ldots$ & $\ldots$ & $\ldots$ \\
\hline 2004 Oct $14^{c}$ & 53292.5 & 96.5 & 160 & $\ldots$ & $\ldots$ & $\ldots$ & $\ldots$ & $\ldots$ & $\ldots$ & $0.74(16)$ \\
\hline $2004 \operatorname{Nov} 1^{c}$ & 53310.1 & 114.1 & 134 & $4.37(2)$ & $7.70(2)$ & $4.78(3)$ & $2.49(3)$ & $\ldots$ & $\ldots$ & $\ldots$ \\
\hline 2004 Nov $6^{c}$ & 53315.7 & 119.7 & 160 & $\ldots$ & $\ldots$ & $\ldots$ & $\ldots$ & $\ldots$ & $\ldots$ & $0.70(9)$ \\
\hline 2005 Mar $3^{c}$ & 53432.3 & 236.3 & 160 & $\ldots$ & $\ldots$ & $\ldots$ & $\ldots$ & $\ldots$ & $\ldots$ & $0.89(9)$ \\
\hline $2005 \operatorname{Mar} 24^{\mathrm{c}}$ & 53453.9 & 257.9 & 134 & $1.25(2)$ & $3.50(1)$ & $1.57(3)$ & $1.27(4)$ & $\ldots$ & $\ldots$ & $\ldots$ \\
\hline $2005 \mathrm{Apr} 2^{\mathrm{c}}$ & 53462.0 & 266.0 & 160 & $\ldots$ & $\ldots$ & $\ldots$ & $\ldots$ & $\ldots$ & $\ldots$ & $0.75(17)$ \\
\hline 2005 Oct $20^{e}$ & 53663.7 & 467.7 & 536 & $1.86(2)$ & $2.36(1)$ & $2.42(2)$ & $2.36(4)$ & $\ldots$ & $\ldots$ & $\ldots$ \\
\hline $2005 \operatorname{Nov} 22^{\mathrm{e}}$ & 53693.9 & 497.9 & 630 & $\ldots$ & $\ldots$ & $\ldots$ & $\ldots$ & $1.23(4)$ & $\ldots$ & $\ldots$ \\
\hline $2006 \operatorname{Mar} 23^{\mathrm{e}}$ & 53817.9 & 621.9 & 536 & $1.34(2)$ & $1.74(1)$ & $2.38(2)$ & $2.58(4)$ & $\ldots$ & $\ldots$ & $\ldots$ \\
\hline 2006 Apr $2^{\text {f }}$ & 53827.6 & 631.6 & 10 & $\ldots$ & $\ldots$ & $\ldots$ & $\ldots$ & $\ldots$ & $\ldots$ & $1.0(2)^{\mathrm{g}}$ \\
\hline 2006 Apr $23^{e}$ & 53848.4 & 652.4 & 630 & $\ldots$ & $\ldots$ & $\ldots$ & $\ldots$ & $1.43(7)$ & $\ldots$ & $\ldots$ \\
\hline 2006 Oct $28^{\mathrm{h}}$ & 54036.2 & 840.2 & 536 & $0.85(2)$ & $1.14(1)$ & $1.81(2)$ & $2.09(4)$ & $\ldots$ & $\ldots$ & $\ldots$ \\
\hline 2006 Oct $31^{\mathrm{i}}$ & 54039.1 & 843.1 & 14 & $0.83(2)$ & $1.13(2)$ & $1.50(7)$ & $2.07(4)$ & $\ldots$ & $\ldots$ & $\ldots$ \\
\hline 2006 Nov $16^{\mathrm{i}}$ & 54054.8 & 859.0 & 57 & $\ldots$ & $\ldots$ & $\ldots$ & $\ldots$ & $1.38(7)$ & $\ldots$ & $\ldots$ \\
\hline 2006 Nov $16^{\mathrm{h}}$ & 54055.0 & 859.0 & 315 & $\ldots$ & $\ldots$ & $\ldots$ & $\ldots$ & $1.47(4)$ & $1.15(3)$ & $\ldots$ \\
\hline $2006 \operatorname{Dec} 1^{i}$ & 54070.3 & 874.3 & 160 & $\ldots$ & $\ldots$ & $\ldots$ & $\ldots$ & $\ldots$ & $\ldots$ & $1.15(15)$ \\
\hline $2007 \operatorname{Mar} 24^{\mathrm{h}}$ & 54183.0 & 987.0 & 1258 & $\ldots$ & $\ldots$ & $\ldots$ & $\ldots$ & $1.28(9)^{\mathrm{j}}$ & $0.96(8)^{j}$ & $\ldots$ \\
\hline $2007 \operatorname{Mar} 24^{\mathrm{i}}$ & 54183.0 & 987.0 & 132 & $\ldots$ & $\ldots$ & $\ldots$ & $\ldots$ & $1.37(10)^{\mathrm{j}}$ & $\ldots$ & $\ldots$ \\
\hline 2007 Apr $2^{\mathrm{h}}$ & 54192.8 & 996.8 & 536 & $0.50(2)$ & $0.67(1)$ & $1.15(2)$ & $1.37(4)$ & $\ldots$ & $\ldots$ & $\ldots$ \\
\hline 2007 Apr $2^{\mathrm{i}}$ & 54192.8 & 996.8 & 125 & $0.49(2)$ & $0.67(1)$ & $1.13(3)$ & $1.38(4)$ & $\ldots$ & $\ldots$ & $\ldots$ \\
\hline 2007 Apr $13^{\mathrm{i}}$ & 54203.1 & 1007.1 & 160 & $\ldots$ & $\ldots$ & $\ldots$ & $\ldots$ & $\ldots$ & $\ldots$ & $0.90(10)$ \\
\hline 2007 Oct $24^{\mathrm{k}}$ & 54397.3 & 1201.3 & 494 & $\ldots$ & $\ldots$ & $\ldots$ & $\ldots$ & $\ldots$ & $\ldots$ & $1.09(13)$ \\
\hline 2007 Oct $24^{1}$ & 54397.3 & 1201.3 & 1360 & $\ldots$ & $\ldots$ & $\ldots$ & $\ldots$ & $\ldots$ & $\ldots$ & $1.07(13)$ \\
\hline $2007 \operatorname{Nov} 4^{k}$ & 54408.3 & 1212.3 & 283 & $\ldots$ & $\ldots$ & $\ldots$ & $\ldots$ & $1.18(4)$ & $\ldots$ & $\ldots$ \\
\hline 2007 Nov $19^{i}$ & 54423.3 & 1227.3 & 322 & $0.36(2)$ & $0.45(1)$ & $0.76(2)$ & $1.01(4)$ & $\ldots$ & $\ldots$ & $\ldots$ \\
\hline 2007 Nov $23^{\mathrm{k}}$ & 54427.1 & 1231.1 & 322 & $0.36(2)$ & $0.44(1)$ & $0.76(2)$ & $1.03(3)$ & $\ldots$ & $\ldots$ & $\ldots$ \\
\hline 2007 Nov $24^{1}$ & 54428.0 & 1232.0 & 3485 & $0.36(1)$ & $0.45(1)$ & $0.80(2)$ & $1.02(3)$ & $\ldots$ & $\ldots$ & $\ldots$ \\
\hline 2007 Nov $29^{i}$ & 54433.4 & 1237.4 & 494 & $\ldots$ & $\ldots$ & $\ldots$ & $\ldots$ & $\ldots$ & $\ldots$ & $1.20(6)$ \\
\hline 2007 Dec $6^{i}$ & 54440.0 & 1244.0 & 283 & $\ldots$ & $\ldots$ & $\ldots$ & $\ldots$ & $1.80(35)^{\mathrm{j}}$ & $\ldots$ & $\ldots$ \\
\hline 2007 Dec $15^{1}$ & 54450.0 & 1254.0 & 1260 & $\ldots$ & $\ldots$ & $\ldots$ & $\ldots$ & $1.12(3)$ & $\ldots$ & $\ldots$ \\
\hline $2008 \operatorname{Mar} 25^{1}$ & 54551.0 & 1355.0 & 1260 & $\ldots$ & $\ldots$ & $\ldots$ & $\ldots$ & $1.03(6)$ & $\ldots$ & $\ldots$ \\
\hline 2008 Apr $7^{1}$ & 54563.8 & 1367.8 & 3485 & $0.33(2)$ & $0.38(1)$ & $0.67(3)$ & $0.90(4)$ & $\ldots$ & $\ldots$ & $\ldots$ \\
\hline $2008 \mathrm{Apr} 12^{\mathrm{k}}$ & 54568.2 & 1372.2 & 322 & $0.33(2)$ & $0.38(1)$ & $0.63(2)$ & $0.95(4)$ & $\ldots$ & $\ldots$ & $\ldots$ \\
\hline 2008 Apr $14^{\mathrm{k}}$ & 54570.4 & 1374.4 & 494 & $\ldots$ & $\ldots$ & $\ldots$ & $\ldots$ & $\ldots$ & $\ldots$ & $1.24(9)$ \\
\hline 2008 Apr $15^{1}$ & 54571.0 & 1375.0 & 1360 & $\ldots$ & $\ldots$ & $\ldots$ & $\ldots$ & $\ldots$ & $\ldots$ & $1.11(14)$ \\
\hline 2008 May $3^{k}$ & 54589.3 & 1393.3 & 283 & $\ldots$ & $\ldots$ & $\ldots$ & $\ldots$ & $1.07(6)$ & $\ldots$ & $\ldots$ \\
\hline 2009 Nov $14^{\mathrm{m}}$ & 55149.9 & 1953.9 & 3276 & $0.31(1)$ & $\ldots$ & $\ldots$ & $\ldots$ & $\ldots$ & $\ldots$ & $\ldots$ \\
\hline 2009 Dec $3^{\mathrm{m}}$ & 55168.7 & 1972.7 & 3276 & $0.30(1)$ & $\ldots$ & $\ldots$ & $\ldots$ & $\ldots$ & $\ldots$ & $\ldots$ \\
\hline 2010 Apr $16^{\mathrm{m}}$ & 55302.7 & 2106.7 & 3276 & $0.35(1)$ & $\ldots$ & $\ldots$ & $\ldots$ & $\ldots$ & $\ldots$ & $\ldots$ \\
\hline 2010 May $23^{\mathrm{m}}$ & 55339.5 & 2143.5 & 3276 & $0.31(1)$ & $\ldots$ & $\ldots$ & $\ldots$ & $\ldots$ & $\ldots$ & $\ldots$ \\
\hline Sandage 96 & & & & $0.28(5)$ & $0.21(4)$ & $0.14(3)$ & $0.08(2)$ & $0.024(7)$ & $0.013(4)$ & $0.011(3)$ \\
\hline
\end{tabular}

Notes.

a All measurements were made in a $\sim 3$ ". 7 radius circular aperture, with annular sky measured between $\times 1.5$ and $\times 2.2$ the aperture radius. The aperture was centered on the WCS coordinates of the SN. Statistical uncertainties in the last one or two significant figures are shown in brackets. The fluxes have not been corrected for reddening, nor for the contribution of the S96 cluster. The last line shows the estimated contribution to the total flux by S96 (see Section 3.1). The exposure times in Column 4 are per band. It can be seen that some same-wavelength observation epochs have negligible temporal spacing as well as differing exposure times (viz. 840/3 days, 859 days, 987 days, 996 days, 1201 days, 1227/31/32, 1244/54, 1367/72, 1374/5). This was due to the impact of Spitzer scheduling constraints on the MISC and SEEDS programs.

${ }^{\mathrm{b}}$ We assume an explosion date of 2004 July 10.0 (MJD = 53196.0).

${ }^{c}$ PID. 00226 Van Dyk (MISC).

d PID. 00159 Kennicutt et al. (SINGS).

e PID. 20256 Meikle et al. (MISC).

${ }^{f}$ PID. 20321 Zaritsky.

$\mathrm{g}$ Target $\sim 50 \%$ off edge of field. Total flux estimated by extrapolation.

${ }^{\text {h PID. }} 30292$ Meikle et al. (MISC).

${ }^{i}$ PID. 30494 Sugerman et al. (SEEDS).

j Contamination to northeast.

${ }^{k}$ PID. 40010 Meixner et al. (SEEDS).

${ }^{1}$ PID. 40619 Kotak et al. (MISC).

${ }^{m}$ PID. 61002 Freedman et al. 


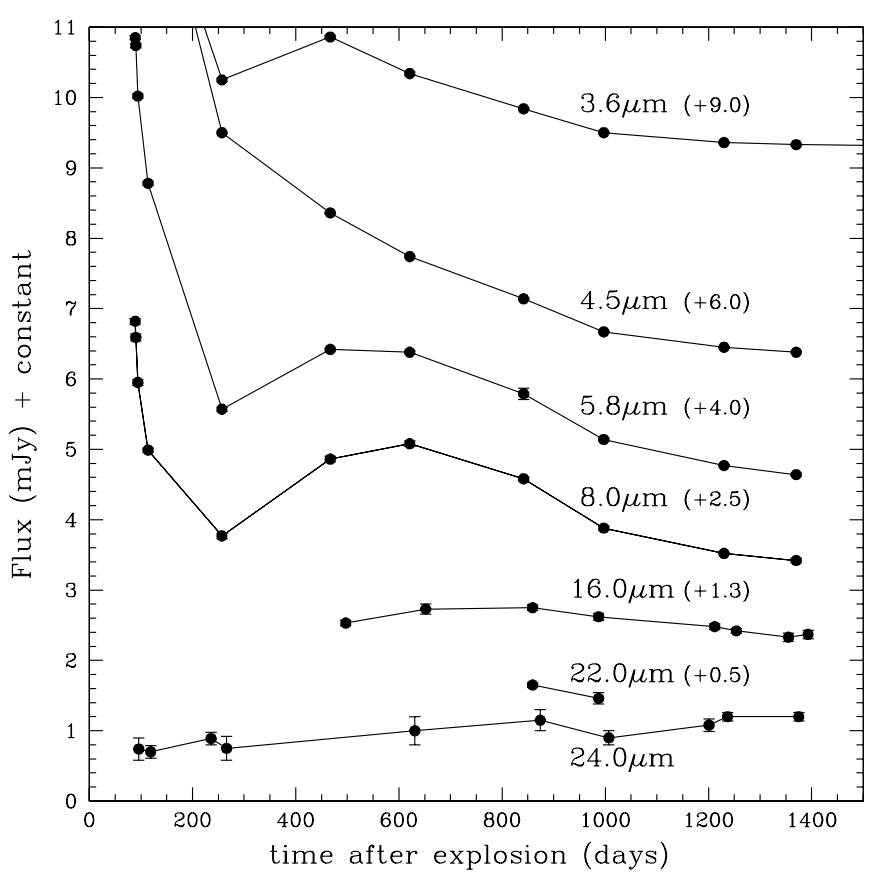

Figure 2. MIR light curves of SN 2004dj. They are uncorrected for reddening. For clarity, the plots have been shifted vertically by the amounts shown in brackets (mJy). Off to the right of the plot there are four additional $3.6 \mu \mathrm{m}$ points spanning 1954 days to 2143 days (Table 1). They show little significant change during this period, with a mean flux of $0.32 \pm 0.02 \mathrm{mJy}$. This is consistent with there having been no decline since 1372 days. The MIR flux of S96 alone has not been measured and so the light curves are uncorrected for S96. An estimate of the S96 contribution is given in Table 1 and is discussed in Section 3.1.

brighter at 621 days (about $\times 2$ relative to 257 days). This is due to the epoch being close to the peak of the thermal emission from the dust (see Section 3.4.4.2). It can also be seen that the $\mathrm{SN}$ lies in a region of relatively bright, complex background emission. The MIR photometry is displayed as light curves and spectral energy distributions (SEDs) in Figures 2 and 3, respectively.

In Figure 2, a rapid initial decline is seen at wavelengths 3.6-8.0 $\mu \mathrm{m}$. With the exception of $4.5 \mu \mathrm{m}$, all of the light curves (with sufficient temporal coverage) exhibit secondary maxima, with the peak emission occurring at epochs of $\sim 450$ days at $3.6 \mu \mathrm{m}$ to $\sim 850$ days at $24 \mu \mathrm{m}$. As will be discussed later, these second maxima constitute strong evidence of dust formation. The absence of a delayed peak at $4.5 \mu \mathrm{m}$ is due to the earlier appearance and dominance of $\mathrm{CO}$ fundamental emission in this band. After $\sim 1000$ days, the $24 \mu \mathrm{m}$ light curve starts to climb again. The evolution of the $24 \mu \mathrm{m}$ flux is complex, as it is a combination of the detailed behaviors of emission from the ejecta dust and from an interstellar (IS) IR echo (see Section 3.4.3.1). In Figure 3, we see a steady reddening of the MIR SED with time. It is argued below (Section 3.4.4.2) that this effect also constitutes strong evidence of dust formation and cooling in the $\mathrm{SN}$ ejecta. The large peak at $4.5 \mu \mathrm{m}$ at 114 days and 257 days is due to the aforementioned dominance of $\mathrm{CO}$ fundamental emission in this band. As in Table 1, neither Figure 2 nor Figure 3 has been corrected for S96.

\subsection{Optical and Near-infrared Photometry}

NIR imaging of SN 2004dj was obtained using Long-slit Intermediate Resolution Infrared Spectrograph (LIRIS) on the $4.2 \mathrm{~m}$ William Herschel Telescope (WHT), La Palma, and at an effectively single epoch (spanning two days) with the OSU-MDM IR Imager/Spectrograph on the $2.4 \mathrm{~m}$ Hiltner

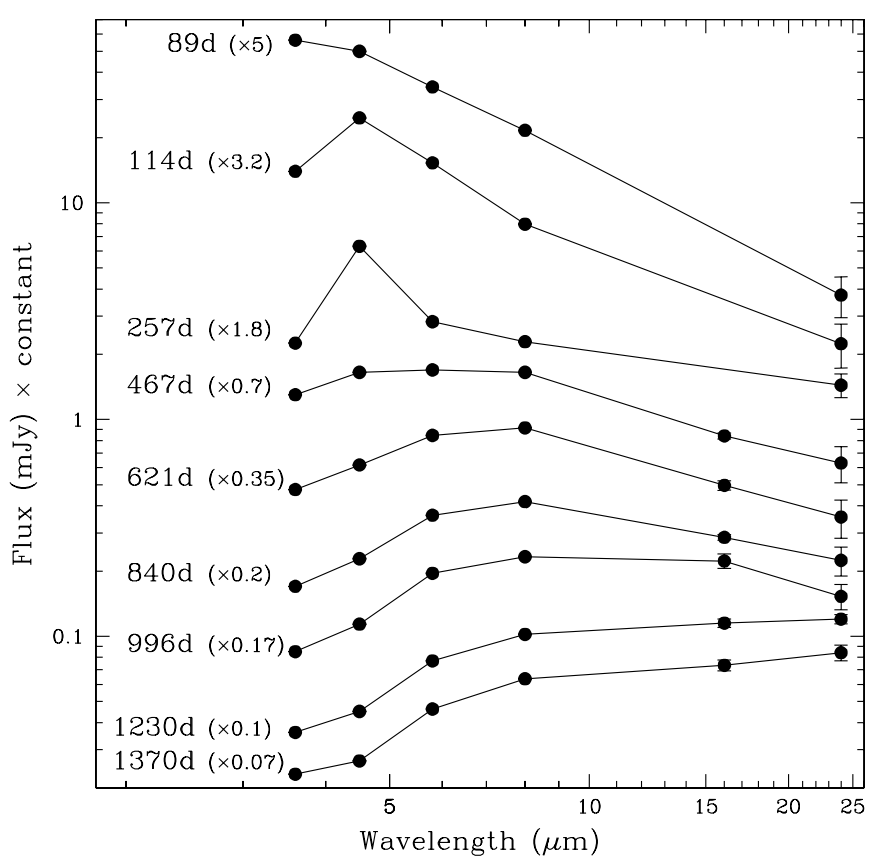

Figure 3. Evolution of the MIR spectral energy distribution of SN 2004dj. The SEDs are uncorrected for reddening. For clarity, the individual SEDs have been scaled by the amounts shown in brackets. As in Figure 2, the fluxes from S96 have not been subtracted. The large peak at $4.5 \mu \mathrm{m}$ present at 114 days and 257 days is due to the dominance of $\mathrm{CO}$ fundamental emission in this band during this period.

Telescope of the MDM Observatory, Arizona. The wavebands are $Z(1.033 \mu \mathrm{m}), J(1.250 \mu \mathrm{m}), H(1.635 \mu \mathrm{m})$, and $K_{s}$ $(2.15 \mu \mathrm{m})$. The LIRIS data were reduced using standard IRAF routines. ${ }^{15}$ The jittered on-source exposures were median combined to form sky frames. In each band, the sky-subtracted frames were then aligned and median combined.

Aperture photometry was performed on the reduced images using the Starlink package GAIA (Draper et al. 2002) with the same aperture and sky annuli as for the MIR photometry. The aperture was centered by centroiding on the sources. The magnitudes at $J, H$, and $K_{s}$ were obtained by comparison with four field stars lying within $\sim 100^{\prime \prime}$ of SN 2004dj. The fieldstar magnitudes were acquired by measurement of Two Micron All Sky Survey (2MASS) images (Skrutskie et al. 1997). For the single Z-band measurement, the magnitude was obtained by comparison with the four field stars with their $J H K_{s}$ SEDs extrapolated to the $Z$ band. The resulting $\mathrm{SN}$ photometric measurements are listed in Table 2 and plotted in Figure 4. Errors shown include uncertainties in the magnitudes of the four 2MASS comparison field stars. Pre-explosion $J H K_{s}$ fluxes of S96 were measured from the 2MASS survey (see Table 2) and used to correct the $J H K_{s}$ light curves. Also shown for comparison is the $3.6 \mu \mathrm{m}$ light curve from the present work. In $H$ and $K_{s}$, the slopes flatten after $\sim 300$ days accompanied by a rise at $3.6 \mu \mathrm{m}$. This is suggestive of radiation from warm, newly forming dust.

Optical photometry was taken from Vinkó et al. (2006, 2009). The optical light curves are displayed in Figure 4 and have been corrected for emission from the S96 cluster using the BVRI

15 IRAF is written and supported by the IRAF programming group at the National Optical Astronomy Observatory (NOAO) in Tucson, Arizona, which is operated by the Association of Universities for Research in Astronomy (AURA), Inc., under cooperative agreement with the National Science Foundation (NSF). 
Table 2

Near-IR Photometry of SN 2004dj

\begin{tabular}{lccccccc}
\hline \hline \multicolumn{1}{c}{$\begin{array}{c}\text { Date } \\
(\mathrm{UT})\end{array}$} & MJD & $\begin{array}{c}\text { Epoch }^{\mathrm{a}} \\
(\text { days })\end{array}$ & $\begin{array}{c}t_{\exp } \\
(\mathrm{s})\end{array}$ & \multicolumn{4}{c}{ Magnitudes $^{\mathrm{b}}$} \\
\cline { 5 - 8 } & & 138.0 & $J(70) H(135)$ & $\ldots$ & $13.56(4)$ & $13.24(4)$ & $K_{s}$ \\
\hline 2004 Nov 25 & 53334.0 & $Z(60) J(60) H(60) K(50)$ & $15.4(1)^{\mathrm{c}}$ & $14.35(4)$ & $14.15(6)$ & $14.13(5)$ \\
2005 Mar 17 & 53446.0 & 250.0 & $J(300) H(225) K s(180)$ & $\ldots$ & $14.88(1)$ & $14.54(2)$ & $14.42(4)$ \\
2005 May 11 & 53501.2 & 305.2 & $J(300) H(225)$ & $\ldots$ & $14.88(1)$ & $14.52(2)$ & $\ldots$ \\
2005 May 12 & 53502.1 & 306.1 & & $\ldots$ & $15.87(5)$ & $15.19(5)$ & $14.75(5)$ \\
2006 Jan 14 & 53749.9 & 553.9 & $J(675) H(1025) K s(1215)$ & $\ldots$ & $15.93(11)$ & $15.70(12)$ & $15.15(14)$ \\
\hline Sandage 96 & & & & $\ldots$ & &
\end{tabular}

Notes. The 2005 May 11, 12 images are from the TIFKAM IR camera on the $2.4 \mathrm{~m}$ Hiltner Telescope of the MDM Observatory. All the other images are from the LIRIS IR imager/spectrograph on the $4.2 \mathrm{~m}$ William Herschel Telescope of the Observatorio del Roque de los Muchachos, La Palma.

${ }^{\text {a }}$ We assume an explosion date of 2004 July 10.0 (MJD = 53196.0).

b All measurements were made in a 3".7 radius circular aperture, with annular sky measured between $\times 1.5$ and $\times 2.2$ the aperture radius. The aperture was centered on the centroid of the SN. The error on the last one or two figures is given in parentheses. Errors shown include uncertainties in the magnitudes of the four 2MASS comparison field stars. The fluxes have not been corrected for reddening, nor for the contribution of the S96 cluster (see Section 3.1). The last line shows magnitudes of S96 measured from 2MASS images (Skrutskie et al. 1997).

${ }^{\mathrm{c}}$ Estimated by extrapolation of $J H K_{S}$ SEDs of calibration field stars to the $Z$ band.

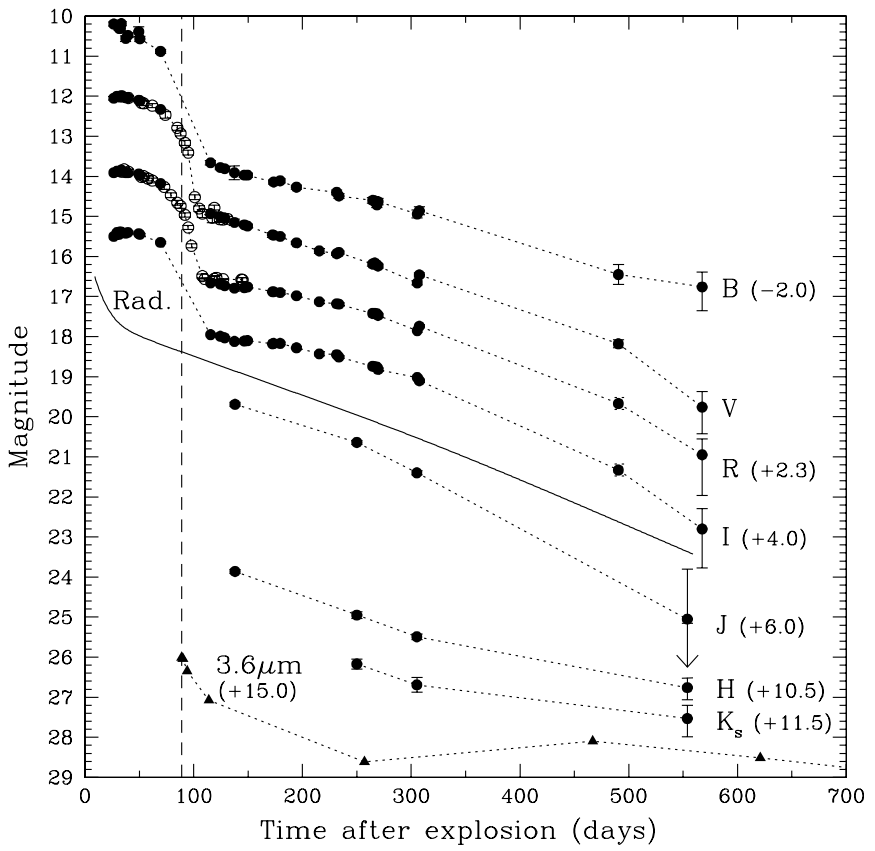

Figure 4. Optical and NIR light curves of SN 2004dj. For clarity, they have been displaced vertically by the values shown in brackets. The optical light curves are from Vinkó et al. (2006, 2009; solid dots). In addition the $V$ and $R$ points of Zhang et al. (2006; open circles) around the end of the plateau have been added to enhance the detail of this phase. For all data sets, the epoch has been adjusted to our explosion epoch of MJD $=53196.0$. The NIR data are from the present work. Also shown is the $3.6 \mu \mathrm{m}$ light curve from the present work. The vertical dashed line is at the epoch of the earliest $3.6 \mu \mathrm{m}$ point and indicates the corresponding phase in the other light curves. S96 fluxes at $B V R I$ and at $J H K_{s}$ have been subtracted from the data. Also shown for comparison (labeled "Rad.") is the temporal evolution of the radioactive energy deposition as specified by $\mathrm{Li}$ et al. (1993) and Timmes et al. (1996) for SN 1987A with the addition of the early-time contribution of ${ }^{56} \mathrm{Ni}$ assuming complete absorption.

magnitudes given by Vinkó et al. (2006). In addition, the $V$ and $R$ points of Zhang et al. (2006) around the end of the plateau were added to enhance the detail of this phase. Also shown for comparison (labeled "Rad.") is the temporal evolution of the radioactive energy deposition for SN 1987A as specified by Li et al. (1993, 0-1200 days) and Timmes et al. (1996, 500-3500 days) with the addition of the early-time contribution of ${ }^{56} \mathrm{Ni}$ decay assuming complete absorption. The radioactive isotopes include ${ }^{56} \mathrm{Ni},{ }^{56} \mathrm{Co},{ }^{57} \mathrm{Co},{ }^{60} \mathrm{Co},{ }^{22} \mathrm{Na}$, and ${ }^{44} \mathrm{Ti}$. (In subsequent use of the $\mathrm{Li}$ et al. and Timmes et al. deposition specifications, our addition of the early-time contribution of ${ }^{56} \mathrm{Ni}$ decay is assumed.) In the optical light curves, for about 35 days (115-150 days) after the end of the fall from the plateau the decline rate matches the radioactive deposition quite closely, indicating that this was powering the emission during this phase. The optical decline rates then flatten during $\sim 150-250$ days, indicating the emergence of an additional power source (see below). After about 250 days, the optical light curves exhibit a steepening (possibly also present in the $J$ band) which increases up to the final observations.

\subsection{Mid-infrared Spectroscopy}

Low-resolution ( $R \approx 60-127$ ) MIR spectroscopy between 5.2 and $14.5 \mu \mathrm{m}$ was acquired at nine epochs between 106.3 days and 1393.3 days with the IRS in low-resolution mode. Long-low (LL; 14-38 $\mu \mathrm{m}$ ) observations were also attempted. Unfortunately, the LL observations were unusable. The LL slit lies at $90^{\circ}$ to the short-low (SL) slit. This meant that, given the scheduling constraints, the LL slit always lays across the host galaxy, resulting in heavy contamination. The MIR spectroscopic observations were drawn from the MISC programs plus one epoch at 1207 days from the SEEDS program. The log of spectroscopic observations is given in Table 3 .

The data were processed through the Spitzer Science Center's pipeline software, which performs standard tasks such as ramp fitting and dark-current subtraction, and produces basic calibrated data (BCD) frames. Starting with these data, we produced reduced spectra using both the SPICE and SMART v6.4 software packages. We first cleaned individual frames of rogue and otherwise "bad" pixels using the IRSCLEAN task. The first and last five pixels, corresponding to regions of reduced sensitivity on the detector, were then removed. The individual frames at each nod position were median combined with equal weighting on each resolution element. Sky background was removed from each combined frame by subtracting the combined frame for the same order taken with the other nod position. We also experimented with background removal by subtracting the adjacent order. In general, nod-nod subtraction was preferred, as 
Table 3

Mid-IR Spectroscopy Log of SN 2004dj

\begin{tabular}{lcccl}
\hline \hline $\begin{array}{c}\text { Date } \\
(\mathrm{UT})\end{array}$ & MJD & $\begin{array}{c}\text { Epoch }^{\mathrm{a}} \\
(\text { days })\end{array}$ & $\begin{array}{c}t_{\text {exp }} \\
(\mathrm{s})\end{array}$ & \multicolumn{1}{c}{ Program } \\
\hline 2004 Oct 24 & 53302.32 & 106.3 & 610 & PID. 00226 Van Dyk (MISC) \\
2004 Nov 16 & 53325.77 & 129.8 & 610 & PID. 00226 Van Dyk (MISC) \\
2005 Mar 18 & 53447.65 & 251.6 & 610 & PID. 00226 Van Dyk (MISC) \\
2005 Apr 17 & 53477.92 & 281.9 & 610 & PID. 00226 Van Dyk (MISC) \\
2005 Nov 22 & 53696.83 & 500.8 & 1828 & PID. 20256 Meikle et al. (MISC) \\
2006 Apr 23 & 53848.47 & 652.5 & 1828 & PID. 20256 Meikle et al. (MISC) \\
2006 Nov 16 & 54055.08 & 859.1 & 3657 & PID. 30292 Meikle et al. (MISC) \\
2007 Oct 30 & 54403.45 & 1207.4 & 117 & PID. 30494 Sugerman et al. (SEEDS) \\
2008 May 3 & 54589.35 & 1393.3 & 3657 & PID. 40619 Kotak et al. (MISC) \\
\hline
\end{tabular}

Notes. Spectral ranges at each epoch were short-low (SL) first and second orders: 7.4-14.5 and 5.2-8.7 $\mu \mathrm{m}$, respectively. $t_{\text {exp }}$ gives the exposure time per order, in seconds.

${ }^{\mathrm{a}}$ We assume an explosion date of 2004 July 10.0 (MJD = 53196.0).

the background sampled in this way is expected to most closely represent the background underlying the SN. Any residual background was removed by fitting low-order polynomials to regions immediately adjacent to the SN position.

One-dimensional spectra were then extracted using the optimal extraction tool within the SPICE software package, with default parameters. We found that in all cases the source was point-like, with a full width at half-maximum (FWHM) intensity that was never wider than the point-spread function (PSF). This procedure results in separate spectra for each nod and for each order. The spectra for each nod were inspected; features present in only one nod were treated as artifacts and removed. The two nod positions were subsequently combined. The nod-combined spectra were then merged to give the final spectrum for each epoch. Overall, we obtained excellent continuum matches between different orders.

Despite our careful reduction procedure, the fluxes of the IRS spectra and the IRAC photometry were not completely consistent. This was due to (1) differences in the fixed sizes of the spectrograph aperture slits and the circular apertures used for the image photometry and (2) the fact that the spectra were generally taken some days before or after the imaging data, during which time the SN flux changed. We therefore recalibrated the IRS spectra against contemporaneous photometry in the $8 \mu \mathrm{m}$ band obtained by interpolation of the light curve. This band was chosen since it was completely spanned by the SL spectrum. For each epoch, the IRAC $8 \mu \mathrm{m}$ transmission function was multiplied by the MIR spectra and by a model spectrum of Vega. ${ }^{16}$ The resulting MIR spectra for the SN and for Vega were integrated over wavelength. The total SN spectral flux in the $8 \mu \mathrm{m}$ band was then obtained from the ratio of the two measurements using a zero (Vega) magnitude of 64.1 Jy (IRAC Data Handbook, Table 5.1). These were then compared with the $8 \mu \mathrm{m}$ photometry to derive scaling factors by which the spectra were multiplied. The spectra are plotted in Figure 5, together with contemporary photometric data.

The MIR spectra comprise both continua and emission features. Up to at least 281 days, strong emission from the CO fundamental was present in the IRS spectra and IRAC photometry. This had disappeared by 500 days. Strong lines of $\mathrm{HI}$, [Ni I], [Ni II], [Co II], and [Ne II] were also present during the first year, but by 500 days only [Ni II] $6.64 \mu \mathrm{m}$, [Ni I] $7.51 \mu \mathrm{m}$, and [Ne II] $12.81 \mu \mathrm{m}$ were still relatively strong

\footnotetext{
16 The R. Kurucz Stellar Atmospheres Atlas, 1993, ftp://ftp.stsci.edu/cdbs/grid/k93models/standards.
}

(see Figure 5). Apart from the CO region during 106-281 days, the $5-14 \mu \mathrm{m}$ region was dominated by continuum emission. Moreover, simple extrapolation below and above the spectral coverage to the limits of the photometric coverage suggests that the continuum dominated over at least 3.6-24 $\mu \mathrm{m}$.

\subsection{Optical and Near-infrared Spectroscopy}

We acquired optical spectra using ISIS on the WHT, La Palma, and DEIMOS (Faber et al. 2003) on the $10 \mathrm{~m} \mathrm{Keck}$ 2 telescope, Hawaii. The 895 days Keck spectrum has already been presented by Vinkó et al. (2009). We also made use of earlier post-plateau optical spectra obtained by Vinkó et al. (2006) at 89 days and 128 days, Leonard et al. (2006) at 95 days, and Chugai et al. (2005) at 100 days. NIR spectroscopy of SN 2004dj was obtained using LIRIS on the WHT and with the OSU-MDM IR Imager/Spectrograph on the $2.4 \mathrm{~m}$ Hiltner Telescope of the MDM Observatory, Arizona. The data were reduced using standard procedures in Figaro (Shortridge 2002) and IRAF. The observing log for the optical and NIR spectra is given in Table 4. The post-100 day spectra are plotted in Figures 6 and 7.

The earlier post-plateau spectra still exhibited pronounced P Cygni features in $\mathrm{H} \alpha$, He I $5876 \AA$ + Na I D, He I $10830 \AA$, He I $20581 \AA$, and O I $7771 \AA$ + K I $7665 / 99 \AA$. By $461 / 467$ days, the absorption components had largely vanished, with a broad-line emission spectrum now being observed. A few lines persisted to as late as the final optical spectroscopy epoch at 925 days. We examined in detail the evolution of the more isolated of these lines, specifically $\mathrm{H} \alpha, \mathrm{Pa} \beta$, [O I] $6300 \AA$, [Fe II] $7155 \AA$, and [Fe II] $12567 \AA$. Table 5 lists the line luminosities (dereddened) versus epoch, together with the radioactive deposition power specified by Li et al. (1993) and Timmes et al. (1996) for SN 1987A, but scaled down to an initial $0.0095 M_{\odot}$ of ${ }^{56} \mathrm{Ni}$ (see Section 3.2). The evolution of the luminosities is plotted in Figure 8. This indicates that from just after the plateau phase to $\sim 460$ days, the $\mathrm{H} \alpha$ and $\mathrm{Pa} \beta$ luminosities declined at a rate roughly comparable to that of the radioactive deposition. In contrast, from their earliest observation at $\sim 300$ days, the [O I] and [Fe II] lines decline significantly more slowly than the radioactive rate. Moreover, by 895 days the summed luminosity of just the $\mathrm{H} \alpha$, [O I] $6300 \AA$, and [Fe II] $7155 \AA$ lines exceeds that of the radioactive input by $\sim 40 \%$, rising to over $60 \%$ by 925 days. Thus, as with the optical light curves, we deduce the appearance of an additional source of energy, possibly earlier than 300 days. 


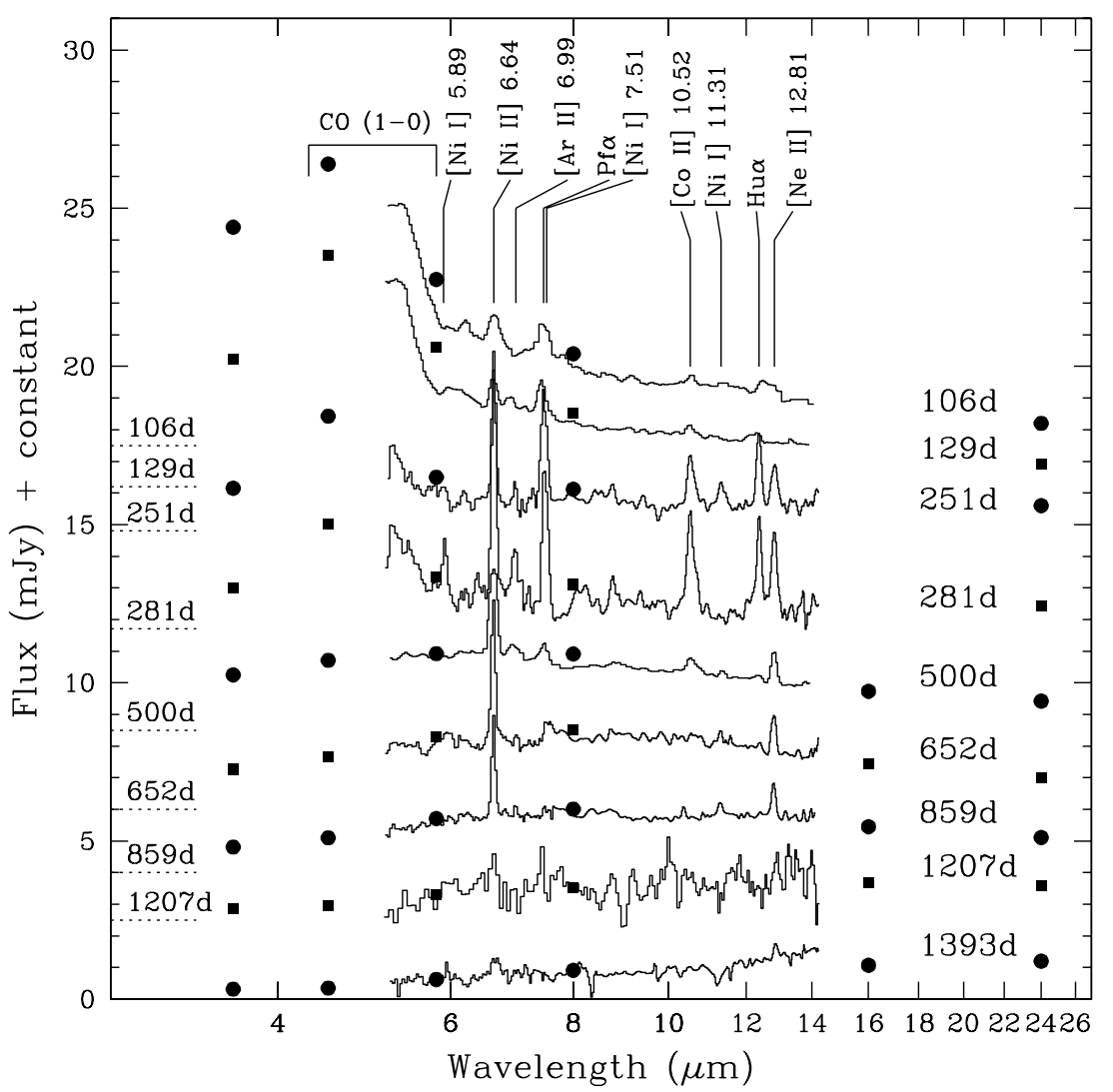

Figure 5. Evolution of the MIR spectra of SN 2004dj. The spectra have not been dereddened and the S96 flux has not been subtracted. No correction has been applied for the heliocentric velocity of the SN. The 1207 days spectrum has a small exposure time and so is of relatively low S/N compared with those of 859 days and 1393 days (see Table 3). Also shown are the fluxes obtained through aperture photometry of the IRAC, PUI, and MIPS images, interpolated to the epochs of the spectra. These photometric data are represented by round or square points, respectively, on alternate epochs. All plots except the latest have been displaced vertically for clarity, with the zero flux levels indicated by the horizontal dotted lines on the left. The stronger features are identified, with the fiducials redshifted to the SN rest frame. The wavelengths are labeled in microns.

Table 4

Optical and Near-infrared Spectroscopy Log of SN 2004dj

\begin{tabular}{llccc}
\hline $\begin{array}{c}\text { Date } \\
(\text { UT })\end{array}$ & MJD & $\begin{array}{c}\text { Epoch }^{\mathrm{a}} \\
(\text { days })\end{array}$ & $\begin{array}{c}\text { Spectral Range } \\
(\mu \mathrm{m})\end{array}$ & Telescope \\
\hline 2004 Oct 5 & 53284.9 & 88.4 & $0.420-0.766$ & DDO $1.88 \mathrm{~m}$ \\
2004 Oct 13 & 53291 & 95 & $0.480-0.910$ & Lick 3 m \\
2004 Oct 18 & 53296 & 100 & $0.370-0.750$ & LAT Scorpio \\
2004 Nov 15 & 53324.21 & 128.2 & $0.402-0.839$ & DDO $1.88 \mathrm{~m}$ \\
2004 Nov 25 & 53334.06 & 138.1 & $0.884-2.406$ & WHT $4.2 \mathrm{~m}$ \\
2005 Apr 19 & 53479.86 & 283.9 & $0.477-0.944$ & WHT $4.2 \mathrm{~m}$ \\
2005 May 12 & 53502.15 & 306.1 & $0.827-2.400$ & Hiltner $2.4 \mathrm{~m}$ \\
2005 Oct 14 & 53657.08 & 461.1 & $0.889-2.453$ & WHT $4.2 \mathrm{~m}$ \\
2005 Oct 20 & 53663.21 & 467.2 & $0.417-0.928$ & WHT $4.2 \mathrm{~m}$ \\
2006 Jan 15 & 53750.03 & 554.0 & $0.890-2.343$ & WHT $4.2 \mathrm{~m}$ \\
2006 Dec 22 & 54091.5 & 895.5 & $0.458-0.723$ & Keck $10 \mathrm{~m}$ \\
2006 Jan 21 & 54121.5 & 925.5 & $0.444-0.890$ & Keck $10 \mathrm{~m}$ \\
\hline
\end{tabular}

Notes. Column 5 details: DDO $1.88 \mathrm{~m}: 1.88 \mathrm{~m}$ telescope of the David Dunlap Observatory (Vinkó et al. 2006). Lick $3 \mathrm{~m}$ : Shane $3 \mathrm{~m}$ telescope at Lick Observatory (Leonard et al. 2006). LAT Scorpio: Scorpio on the $6 \mathrm{~m}$ large azimuthal telescope (Chugai et al. 2005; Chugai 2006). WHT $4.2 \mathrm{~m}: 4.2 \mathrm{~m}$ William Herschel Telescope of the Observatorio del Roque de los Muchachos. Slit width = 1". Hiltner 2.4 m: 2.4 m Hiltner Telescope of the MDM Observatory. Keck 10 m: $10 \mathrm{~m}$ telescope of the W. M. Keck Observatory.

${ }^{\mathrm{a}}$ We adopt an explosion date of 2004 July $10.0(\mathrm{MJD}=53196.0)$.

Table 6 lists profile parameters expressed as velocities for the more isolated lines over a range of epochs, shifted to the center-of-mass rest frame of SN 2004dj. Also listed (Column 7) for 461-925 days are the maximum blue-wing velocities derived from profile model matches (see Section 3.4.4.1). Preliminary inspection indicated that for lines within a given element (e.g., $\mathrm{H} \alpha$ and $\mathrm{Pa} \beta$ ), the velocities exhibited similar values and evolution. Therefore, in order to improve the temporal coverage and sampling, $\mathrm{H} \alpha$ and $\mathrm{Pa} \beta$ were grouped together, as were [Fe II] $7155 \AA$ and [Fe II] $12570 \AA$. The evolution of the line velocities (shifted to the center-of-mass rest frame of the $\mathrm{SN}$ ) is plotted in Figure 9, and in more 
Table 5

Optical and Near-infrared Line Fluxes, $F$, and Luminosities, $L$

\begin{tabular}{|c|c|c|c|c|c|c|c|c|c|c|c|}
\hline \multirow[t]{2}{*}{ Epoch (days) } & \multicolumn{2}{|c|}{$\mathrm{H} \alpha$} & \multicolumn{2}{|c|}{$\mathrm{Pa} \beta$} & \multicolumn{2}{|c|}{ [O I] $6300 \AA$} & \multicolumn{2}{|c|}{$[\mathrm{Fe}$ II $] 7155 \AA$} & \multicolumn{2}{|c|}{ [Fe II $] 12570 \AA$} & \multirow[t]{2}{*}{$L_{\mathrm{rad}}$} \\
\hline & $F$ & $L$ & $F$ & $L$ & $F$ & $L$ & $F$ & $L$ & $F$ & $L$ & \\
\hline 89 & $5300^{\mathrm{a}}(260)$ & $63(3)$ & $\ldots$ & $\ldots$ & . & .. & . . & $\ldots$ & $\ldots$ & $\ldots$ & 586.9 \\
\hline 128 & $2120^{\mathrm{a}}(100)$ & $25(1)$ & $\ldots$ & $\ldots$ & $\ldots$ & $\ldots$ & $\ldots$ & $\ldots$ & $\ldots$ & $\ldots$ & 417.2 \\
\hline 138 & $\ldots$ & $\ldots$ & $380(20)$ & $4.5(2)$ & $\ldots$ & $\ldots$ & $\ldots$ & $\ldots$ & $\ldots$ & $\ldots$ & 381.4 \\
\hline 283 & $1535(80)$ & 18.1(9) & $\ldots$ & $\ldots$ & $250(10)$ & $3.0(2)$ & $82(14)$ & $1.0(2)$ & $\ldots$ & $\ldots$ & 101.7 \\
\hline 306 & $\ldots$ & $\ldots$ & $137(7)$ & $1.62(8)$ & $\ldots$ & $\ldots$ & $\ldots$ & $\ldots$ & $35(10)$ & $0.4(1)$ & 81.5 \\
\hline 461 & & $\ldots$ & $39(4)$ & $0.46(5)$ & $\ldots$ & $\ldots$ & $\ldots$ & $\ldots$ & $20(2)$ & $0.24(2)$ & 16.4 \\
\hline 467 & $230(10)$ & $2.75(15)$ & $\ldots$ & $\ldots$ & $134(7)$ & $1.58(8)$ & $41(7)$ & $0.49(8)$ & $\ldots$ & $\ldots$ & 15.35 \\
\hline 554 & $\ldots$ & $\ldots$ & $9.7(8)$ & $0.11(1)$ & $\ldots$ & $\ldots$ & $\ldots$ & $\ldots$ & $12(3)$ & $0.14(3)$ & 6.0 \\
\hline 895 & $10.7(7)$ & $0.13(1)$ & $\ldots$ & $\ldots$ & $7.6(9)$ & $0.09(1)$ & $3.0(2)$ & $0.035(3)$ & $\ldots$ & $\ldots$ & 0.18 \\
\hline 925 & $9.5(7)$ & $0.12(1)$ & $\ldots$ & $\ldots$ & $6.9(3)$ & $0.082(4)$ & $2.52(8)$ & $0.031(1)$ & $\ldots$ & $\ldots$ & 0.14 \\
\hline
\end{tabular}

Notes. Line fluxes, $F$, are in units of $10^{-15} \mathrm{erg} \mathrm{cm}^{-2} \mathrm{~s}^{-1}$, while dereddened line luminosities, $L$, are in units of $10^{38} \mathrm{erg} \mathrm{s}^{-1}$. Figures in brackets give the error on the last one or more significant figures. The errors in the fluxes and luminosities are primarily due to uncertainties in the absolute fluxing and the levels of the underlying continua. Not included in the luminosity errors are systematic uncertainties in the distance and extinction (see Section 1.1). ${ }^{a}$ Spectra from Vinkó et al. (2006). In the final column is shown $L_{\text {rad }}$, the radioactive deposition power corresponding to the ejection of $0.0095 M_{\odot}$ of ${ }^{56} \mathrm{Ni}$, scaled from the SN 1987A case specified by Li et al. (1993) and Timmes et al. (1996).

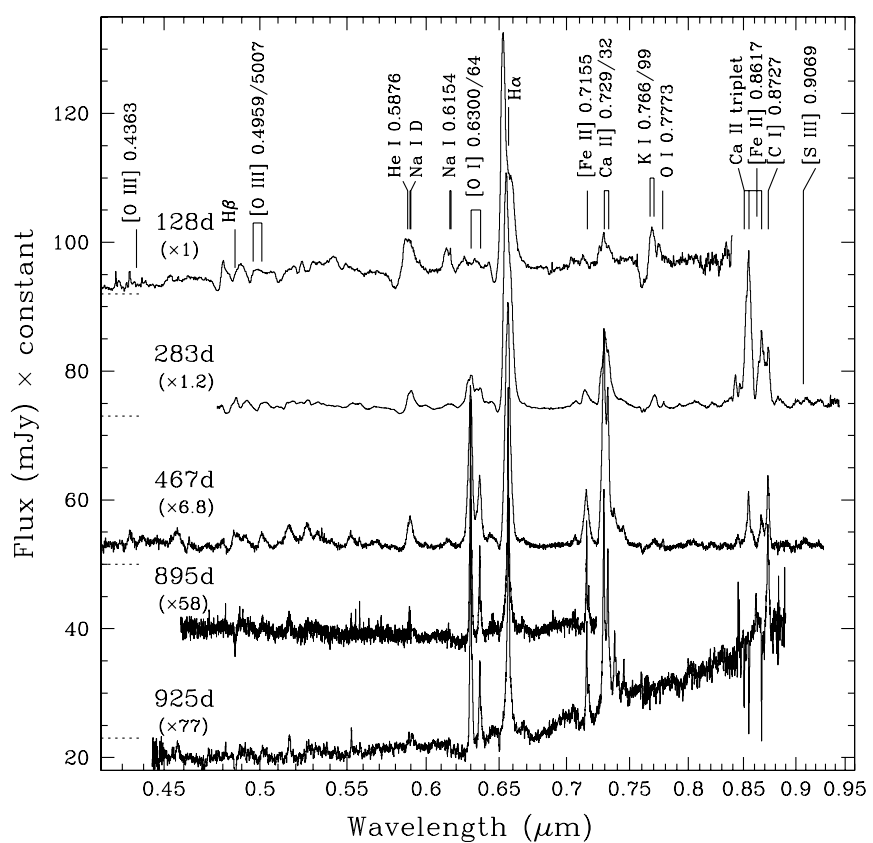

Figure 6. Evolution of the optical spectra of SN 2004dj. The 128 days spectrum is from Vinkó et al. (2006) and the 895 days spectrum has already been presented in Vinkó et al. (2009). The spectra have not been dereddened. In addition, the S96 flux has not been subtracted and this accounts for the relatively large continuum fluxes at epochs 895 days and 925 days. No correction has been applied for the heliocentric velocity of the SN. All plots except the latest have been displaced vertically for clarity, with the zero flux levels indicated by the horizontal dotted lines on the left. Note that the zero flux shown at $23 \mathrm{mJy}$ corresponds to the 895 days spectrum. Also, the spectra have been flux scaled by the amounts shown in brackets. Locations of spectral lines of interest are identified with the fiducials redshifted to the SN rest frame. The wavelengths are labeled in microns.

detail in Figure 10. These plots reveal a complex velocity evolution.

Up to 138 days, of the three elements considered, only hydrogen lines could be reliably identified. At 89 days (corresponding to about half-way down the plateau-edge), the half-width at half-maximum (HWHM) velocity was $1820 \pm 60 \mathrm{~km} \mathrm{~s}^{-1}$, although the red and blue wings extended to much higher values. In addition, the peak emission exhibited a blueshift of $-450 \pm 50 \mathrm{~km} \mathrm{~s}^{-1}$. Then, as already described in Section 1,

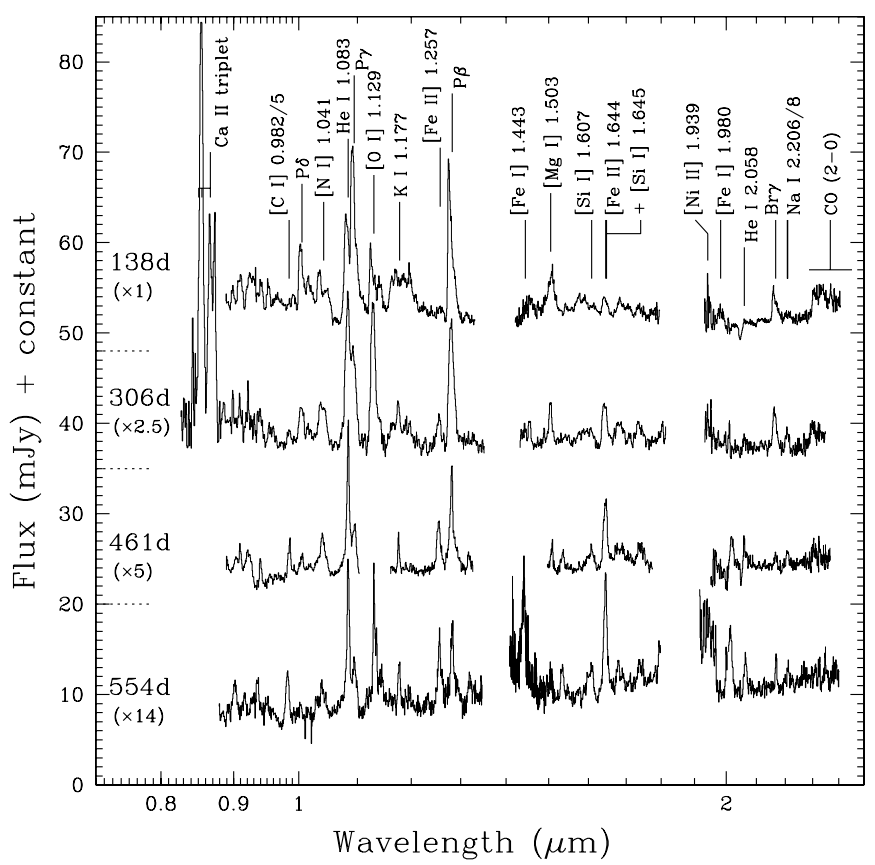

Figure 7. Evolution of the NIR spectra of SN 2004dj. The spectra have not been dereddened and the S96 flux has not been subtracted. No correction has been applied for the heliocentric velocity of the SN. All plots except the latest have been displaced vertically for clarity, with the zero flux levels indicated by the horizontal dotted lines on the left. Also, the spectra have been flux scaled by the amounts shown in brackets. The stronger features are identified with the fiducials redshifted to the SN rest frame. The wavelengths are labeled in microns.

a strong asymmetry rapidly developed, this being attributed to the emergence of an asymmetric, bipolar core (Chugai 2006). Rather than being entirely due to the bulk motion of the ejecta, however, some of the width of the $\mathrm{H} \alpha$ and $\mathrm{Pa} \beta$ lines may also have been produced by scattering from thermal electrons as in the cases of SN 1998S (Chugai 2001) and SN 2006gy (Smith et al. 2010), but this effect is unlikely to have a significant influence at later epochs. By the time of the next observation at 283 days, the asymmetry had diminished, with the blueshift of the peak now only $-730 \pm 50 \mathrm{~km} \mathrm{~s}^{-1}$. By this time the lines of $\left[\mathrm{O}_{\mathrm{I}}\right]$ and $[\mathrm{Fe} \mathrm{II}]$ had emerged, also with asymmetric blueshifted 


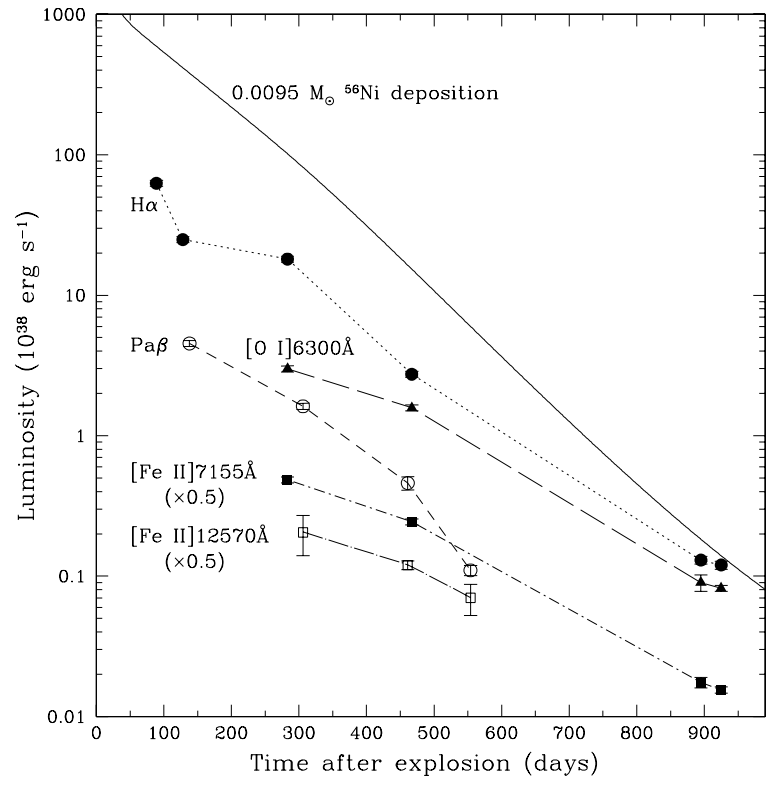

Figure 8. Evolution of spectral line luminosities of SN 2004dj. The luminosities have been corrected for extinction. Also shown is the deposition power of $0.0095 M_{\odot}$ of $56 \mathrm{Ni}$, scaled from the SN 1987A case described by Li et al. (1993) and Timmes et al. (1996). The errors in the fluxes and luminosities are primarily due to uncertainties in the absolute fluxing and the levels of the underlying continua. Not included in the luminosity errors are systematic uncertainties in the distance and extinction (see Section 1.1).

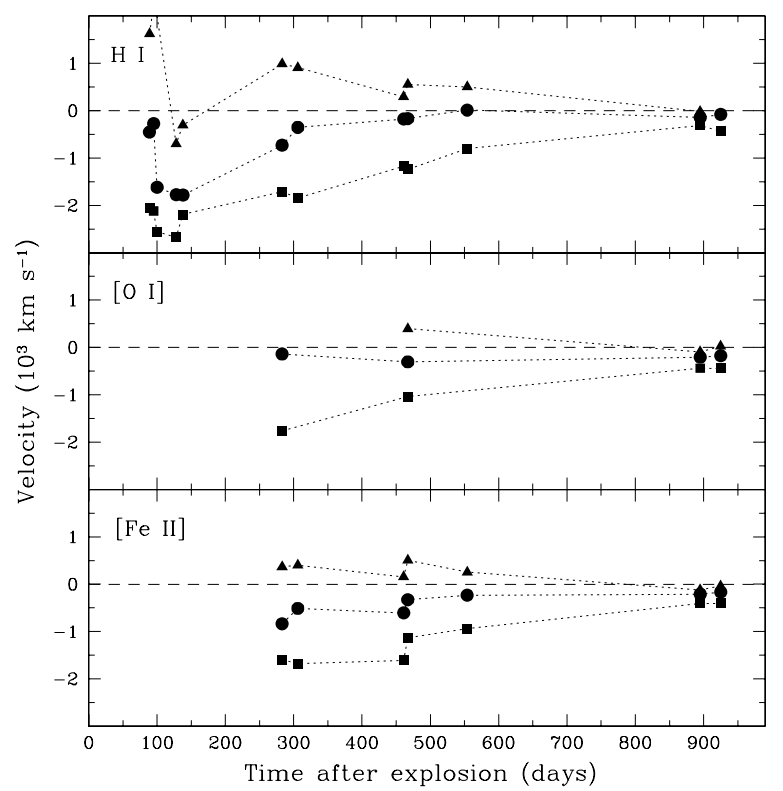

Figure 9. Evolution of spectral line velocities of SN 2004dj (shifted to the center-of-mass rest frame of the $\mathrm{SN}$ ). The plots are as follows: triangles = red wings, half-width half-maximum, round dots $=$ peak intensity, squares $=$ blue wings, and half-width half-maximum. On 895 days and 925 days, the red wing half-width half-maximum refers to the blueshifted component only. Typical uncertainties in the period 89-554 days, in $\mathrm{km} \mathrm{s}^{-1}$, are \pm 100 (RHM), \pm 50 (peak), and \pm 50 (BHM). At 895 days and 925 days, the uncertainties are smaller by a factor of $\sim 4$.

profiles. As the $\mathrm{SN}$ continued to evolve, the line widths narrowed and by 461 days we see the first signs of a sharp suppression of the red wing. By the time of the next season's observations (895 days, 925 days), this suppression is very pronounced in all three species. This phenomenon suggests dust formation resulting in the obscuration of the far side of the ejecta and will be examined in more detail in Section 3.4.4.1.
Table 6

Optical and Near-infrared Line Profile Parameters

\begin{tabular}{|c|c|c|c|c|c|c|}
\hline \multirow{2}{*}{$\begin{array}{l}\text { Epoch } \\
\text { (days) } \\
\end{array}$} & \multicolumn{4}{|c|}{ Velocity $\left(\mathrm{km} \mathrm{s}^{-1}\right)$} & \multirow{2}{*}{$\begin{array}{c}R_{\mathrm{BHM}} \\
\left(10^{15} \mathrm{~cm}\right)\end{array}$} & \multirow{2}{*}{$\begin{array}{r}\text { Model } \\
\left(\mathrm{km} \mathrm{s}^{-1}\right)\end{array}$} \\
\hline & $v_{\mathrm{BHM}}$ & $v_{\text {peak }}$ & $v_{\text {RHM }}$ & $v_{\text {HWHM }}$ & & \\
\hline \multicolumn{7}{|c|}{$\mathrm{H} \alpha$ and $\mathrm{Pa} \beta$} \\
\hline $89^{a}$ & -2010 & -450 & 1630 & 1820 & 1.5 & $\ldots$ \\
\hline $95^{\mathrm{b}}$ & -2120 & -270 & 2210 & 2160 & 1.7 & $\ldots$ \\
\hline $100^{c}$ & -2550 & -1620 & 2000 & 2270 & 2.2 & $\ldots$ \\
\hline $128^{\mathrm{a}}$ & -2670 & -1770 & -700 & 980 & 2.9 & $\ldots$ \\
\hline 138(NIR) & -2190 & -1780 & -310 & 940 & 2.6 & $\ldots$ \\
\hline 283 & -1710 & -730 & 990 & 1350 & 4.2 & $\ldots$ \\
\hline 306(NIR) & -1840 & -350 & 910 & 1370 & 4.9 & $\ldots$ \\
\hline 461(NIR) & -1170 & -180 & 300 & 730 & 4.7 & -2400 \\
\hline 467 & -1240 & -160 & 550 & 890 & 5.0 & -2400 \\
\hline 554(NIR) & -800 & 15 & 500 & 650 & 3.8 & -2400 \\
\hline 895 & -310 & $-140,160$ & $-10^{\mathrm{d}}$ & $150^{\mathrm{d}}$ & 2.4 & -2400 \\
\hline 925 & -430 & $-80,210$ & $\ldots \mathrm{e}$ & $\ldots$ & 3.4 & -2400 \\
\hline \multicolumn{7}{|c|}{$[\mathrm{O}$ I $] 6300 \AA$} \\
\hline 283 & -1760 & -140 & $\ldots$ & $\ldots$ & 4.3 & $\ldots$ \\
\hline 467 & -1040 & -300 & 400 & 720 & 4.5 & -2250 \\
\hline 895 & -440 & $-210,170$ & $-90^{\mathrm{d}}$ & $170^{\mathrm{d}}$ & 3.4 & -990 \\
\hline 925 & -440 & $-170,210$ & $20^{\mathrm{d}}$ & $230^{d}$ & 3.5 & -990 \\
\hline \multicolumn{7}{|c|}{$[\mathrm{Fe}$ II $] 7155 \AA \& 12570 \AA$} \\
\hline 283 & -1600 & -830 & 370 & 980 & 3.9 & $\ldots$ \\
\hline 306(NIR) & -1670 & -510 & 400 & 1030 & 4.4 & $\ldots$ \\
\hline 461(NIR) & -1610 & -600 & 160 & 880 & 6.4 & -3000 \\
\hline 467 & -1130 & -330 & 510 & 820 & 4.6 & -2250 \\
\hline 554(NIR) & -940 & -230 & 260 & 600 & 4.5 & -2250 \\
\hline 895 & -400 & $-210,490$ & $-120^{\mathrm{d}}$ & $140^{\mathrm{d}}$ & 3.1 & -780 \\
\hline 925 & -410 & $-170,480$ & $-30^{\mathrm{d}}$ & $190^{\mathrm{d}}$ & 3.3 & -900 \\
\hline
\end{tabular}

Notes. All the velocities are with respect to the center-of-mass rest frame of SN 2004dj. Velocity subscripts: BHM: blue wing, half-maximum. RHM: red wing, half-maximum. HWHM: half-width, half-maximum. $R_{\mathrm{BHM}}$ is the product of $v_{\mathrm{BHM}}$ and the elapsed time (epoch).

${ }^{a}$ Vinkó et al. (2006).

${ }^{\mathrm{b}}$ Leonard et al. (2006).

c Chugai et al. (2005, NIR): indicates NIR lines $\mathrm{Pa} \beta$ and [Fe II] $12570 \AA \AA$. In Column 3, 895 days and 925 days, the velocities of the stronger, blueshifted peak, and the weaker, redshifted peak are given (see Sections 2.4 and 3.4.4.1).

${ }^{\mathrm{d}}$ Based on blueshifted component only.

e Blueshifted component insufficiently resolved. Typical uncertainties in the period 89-554 days, in $\mathrm{km} \mathrm{s}^{-1}$, are \pm 50 (BHM), \pm 50 (peak), \pm 100 (RHM), and \pm 60 (HWHM). At 895 days and 925 days, the uncertainties are smaller by a factor of $\sim 4$. The last column, "Model," gives the maximum velocity derived from profile (blue wing) matches $(\delta=0)$-see Section 3.4.4.1.

By 895 days and 925 days, a second, weaker peak redshifted by $\sim 170 \mathrm{~km} \mathrm{~s}^{-1}$ had also appeared in the $\mathrm{H} \alpha$ and [O I] $6300 \AA$ profiles (Figure 10). (The redshifted peak can also be seen in the weaker [O I] $6364 \AA$ component lying at $\sim+3000 \mathrm{~km} \mathrm{~s}^{-1}$ in the [O I] $6300 \AA$ A rest-frame plots.) The previous observations of these lines were at 467 days when the line luminosities were about a factor of 20 greater (Table 5) as well as being much wider (Table 6). Therefore an underlying, weaker, redshifted component could have been present at 467 days or earlier, but was swamped by the main component of the line. The 895 days spectrum has a blueshift in the main peak in $\mathrm{H} \alpha$ of $-140 \pm$ $10 \mathrm{~km} \mathrm{~s}^{-1}$ with the minor peak showing a redshift of about $+160 \pm 10 \mathrm{~km} \mathrm{~s}^{-1}$. In [O I] $6300 \AA$, the corresponding velocities are, respectively, $-210 \pm 10 \mathrm{~km} \mathrm{~s}^{-1}$ and $+170 \pm 10 \mathrm{~km} \mathrm{~s}^{-1}$. Thus, the main (blueshifted) peak and the weaker (redshifted) peak lie roughly symmetrically about the local zero velocity. This suggests that a minor fraction of the line flux originates in an emission zone centered on the SN and having the geometry 


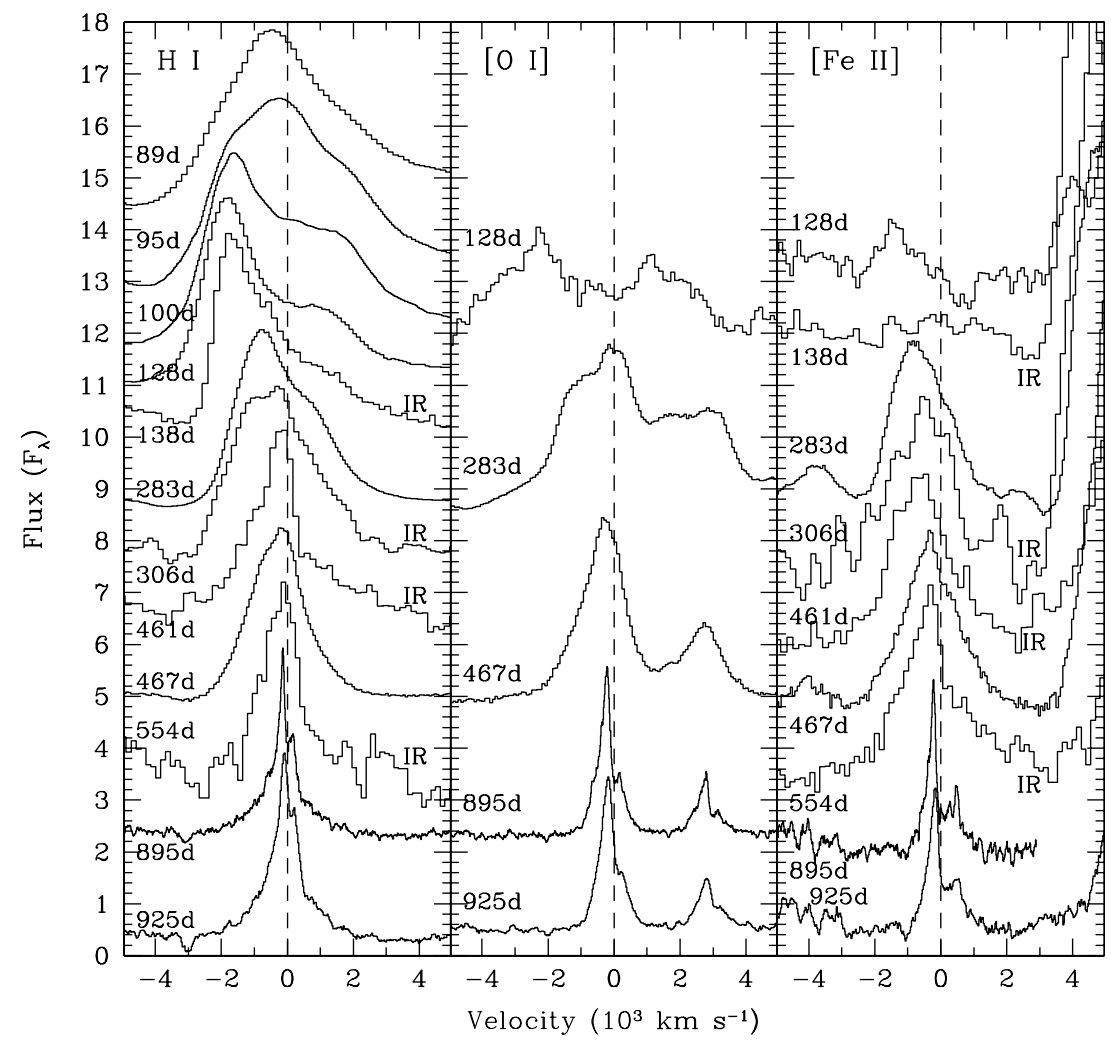

Figure 10. Evolution of the spectral line profiles of SN 2004dj. The 89 and 128 day spectra are from Vinkó et al. (2006), while the 95 and 100 day spectra are from, respectively, Leonard et al. (2006) and Chugai et al. (2005). All other spectra are from the present work. The horizontal axes are in terms of equivalent velocity with respect to the SN center of mass, which has a velocity of $+221 \mathrm{~km} \mathrm{~s}^{-1}$ relative to the Earth. The LH panel shows $\mathrm{H} \alpha$ and $\mathrm{Pa} \beta$ profiles. The Pa $\beta$ profiles are labeled "IR." The middle panel shows [O I] $6300 \AA$ ([O I] $6364 \AA$ can also be seen lying at $\sim+3000 \mathrm{~km} \mathrm{~s}^{-1}$ in the [O I] $6300 \AA$ rest-frame plots). The RH panel shows [Fe II] $7155 \AA$ and [Fe II] $12567 \AA$ profiles. The [Fe II] $12567 \AA$ A profiles are labeled "IR." All the profiles have been flux scaled and shifted vertically for clarity.

of an expanding ring, jet, or cone. The [Fe II] $7155 \AA$ line also shows a secondary peak but at a much larger redshift of $+480 \pm 10 \mathrm{~km} \mathrm{~s}^{-1}$. There may actually be a peak also at around $+170 \mathrm{~km} \mathrm{~s}^{-1}$, similar to those seen in $\mathrm{H} \alpha$ and [O I] $6300 \AA$, but which is swamped by the stronger peak at the larger redshift. The profiles are analyzed in Section 3.4.4.1.

We do not consider the MIR line-profile kinematics due to the much lower resolution $\left(R_{\mathrm{FWHM}} \approx 100\right)$. The formation and evolution of the MIR lines will be analyzed in a future paper.

\section{ANALYSIS}

The evolution of the MIR spectral continuum indicates IR emission from dust playing a major role in the post-plateau flux distribution of SN 2004dj. We now make use of the observations described above to explore the origin, location, distribution, energy source(s), and nature of these grains. This will be done by comparison of a variety of simple models with the observations.

\subsection{Correction of the Supernova Flux for $S 96$}

The position of SN 2004dj coincides with that of the compact star cluster S96 (Sandage 1984), and it seems likely that the progenitor was a member (Wang et al. 2005). Regardless of whether or not this is the case, it is still important to correct for the contribution of the cluster to the photometry and spectra, especially at the later epochs, before we embark on modeling the observed SED. To do this, in the optical region we made use of the pre-explosion optical photometry compiled in Table 4 of Vinkó et al. (2009). NIR photometry of S96 was obtained from 2MASS (see Table 2). Unfortunately, there are no pre-explosion
MIR images of S96 and so its contribution to the flux had to be assessed indirectly.

We found that longward of $\sim 0.4 \mu \mathrm{m}$ the optical/NIR photometric points could be fairly represented by a combination of two blackbodies, reddened according to the Cardelli et al. (1989) law with $E(B-V)=0.1 \mathrm{mag}$ and $R_{V}=3.1$. This is illustrated in Figure 11. In this representation, the fluxes longward of $\sim 1 \mu \mathrm{m}$ are dominated by a component with a temperature of $3500 \mathrm{~K}$. At shorter wavelengths, the hotter component $(50,000 \mathrm{~K})$ becomes increasingly important. This hot blackbody is not intended as an explanation for the short-wave radiation, but rather it simply serves as a means of representing and extrapolating the optical SED. The contribution of S96 to the MIR photometric points was then obtained by extrapolation of the cooler blackbody. (We did not make use of the Vinkó et al. (2009) models to correct for S96 as it was unclear how they should be extrapolated into the MIR region.) Some support for the effectiveness of our estimation method, at least for the shorter wavelengths, comes from four serendipitous $3.6 \mu \mathrm{m}$ images of the SN 2004dj field spanning 1954-2143 days (Table 1), obtained in Spitzer program 61002 (PI: W. Freedman). These show that, by this period, the light curve at this wavelength had leveled out at a mean value of $0.32 \pm 0.02 \mathrm{mJy}$ which in good agreement with our estimate for S96 of $0.28 \pm 0.05 \mathrm{mJy}$ (see Figure 2).

It is possible that the above procedure could underestimate a contribution from cooler material but it is unlikely that S96 would be the source of such emission. Maíz-Apellániz et al. (2004) estimate a cluster age of $13.6 \mathrm{Myr}$ and point out that by this age its parent molecular cloud should have been dispersed by stellar winds and SN explosions. Wang et al. (2005) find an 


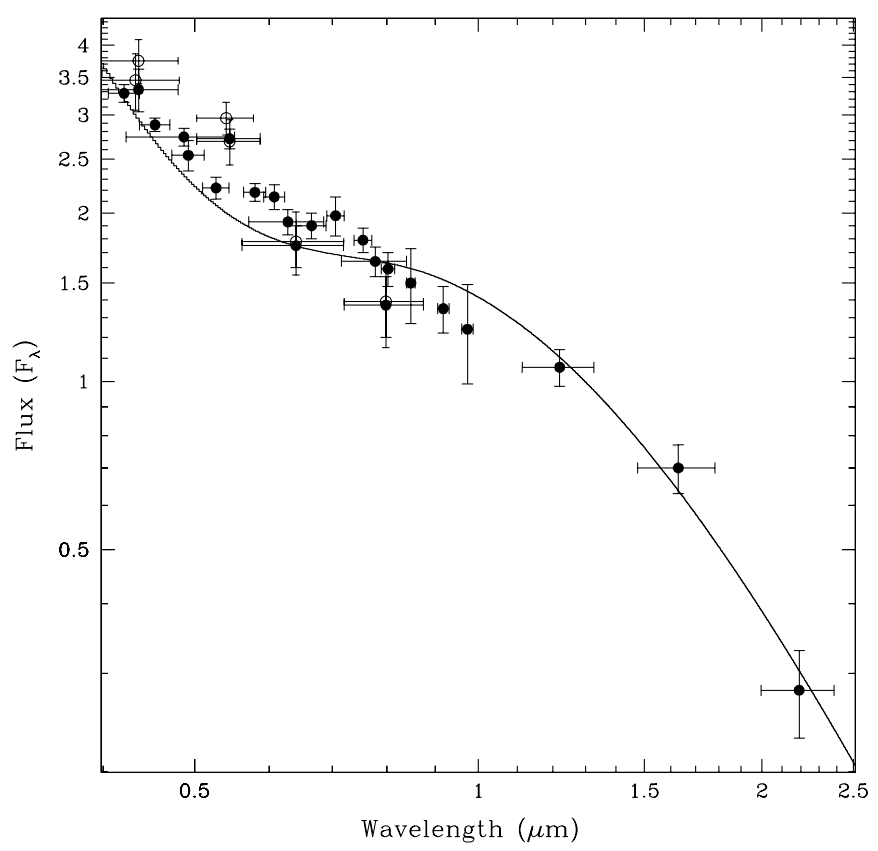

Figure 11. Two-blackbody representation of the S96 SED. The solid and open dots represent the pre-explosion and post-explosion fluxes, respectively (Vinkó et al. 2009). The latter data were acquired at 800 days. In the model, the fluxes longward of $\sim 1 \mu \mathrm{m}$ are dominated by the cold component, with a temperature of $3500 \mathrm{~K}$. The temperature of the hotter component, $50,000 \mathrm{~K}$, is not intended to have a particular physical meaning, but simply serves as a means of representing and extrapolating the optical SED.

age of $\sim 20$ Myr while Vinkó et al. (2009) obtain $\sim 10-16$ Myr. This suggests that the flux contribution from S96 to longer MIR wavelengths would be small. On the other hand, a significant SN-driven IR echo from the general IS dust of the host galaxy is quite likely (see Section 3.4.3.1).

We conclude that the $3500 \mathrm{~K}$ blackbody extrapolation provides a reasonable estimate of the MIR flux contributions from S96. The inferred S96 fluxes in the 3.6-24 $\mu \mathrm{m}$ range are shown in Table 1. The values are insensitive to the extinction over the ranges of $E(B-V)(0.06-0.35 \mathrm{mag})$ suggested in the literature. It can be seen that the contribution of S96 at $3.6 \mu \mathrm{m}$ is significant as early as $\sim 250$ days and dominates by $\sim 1000$ days. As we move to longer wavelengths, the effect of S96 declines, becoming negligible for wavelengths longward of $\sim 10 \mu \mathrm{m}$ even at the latest epochs. In the optical-NIR-MIR continuum modeling (see Section 3.4.4.2), we use the S96 blackbody representation to correct for its contribution to the SED.

\subsection{Mass of ${ }^{56} \mathrm{Ni}$ in the Ejecta}

It is important to establish the mass of ${ }^{56} \mathrm{Ni}$ in the ejecta of SN 2004dj since this will allow us to test for the presence of energy sources, other than radioactive decay, which might be responsible for the SN luminosity. In Figure 12, we show the bolometric light curves (BLCs) of Vinkó et al. (2006, open circles) and Zhang et al. (2006, open triangles). The phases of these have been shifted to our adopted explosion date of $\mathrm{MJD}=53196.0$. This date is nearly one month later than that of Zhang et al. reducing their derived ${ }^{56} \mathrm{Ni}$ mass by about $25 \%$. The explosion date of Vinkó et al. is about 10 days earlier than ours but it is not clear if this would significantly affect the ${ }^{56} \mathrm{Ni}$ mass they derived. In addition to the phase shifts, the BLCs of these authors have been scaled downward to our adopted distance of $3.13 \mathrm{Mpc}$. This has the effect of reducing the ${ }^{56} \mathrm{Ni}$ masses of

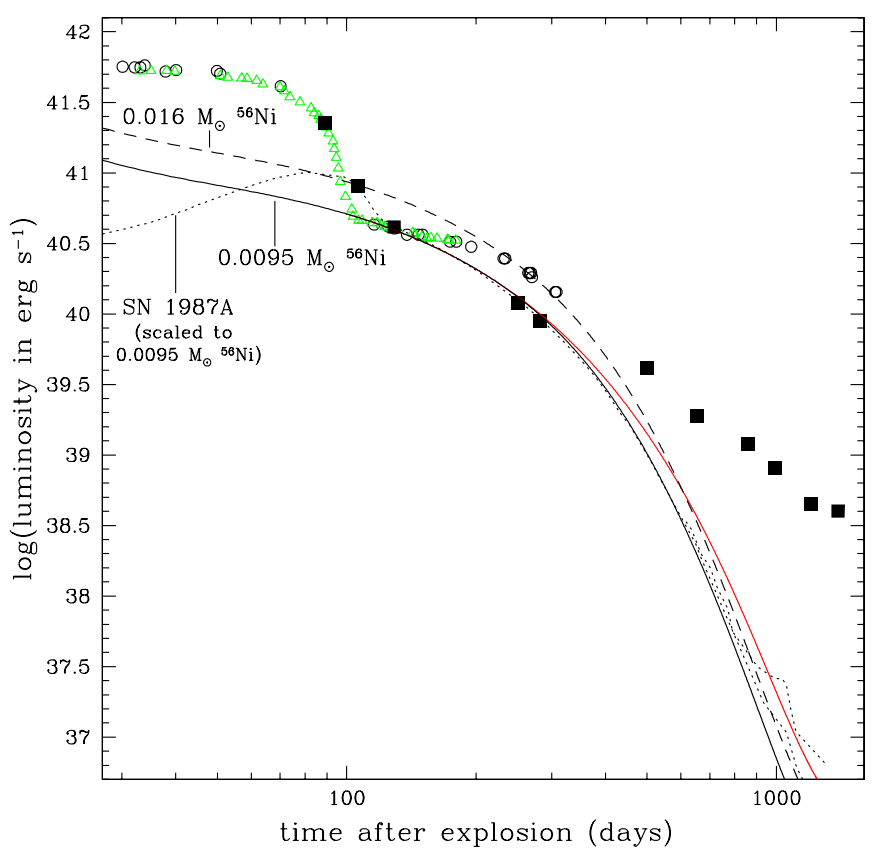

Figure 12. BLCs of Vinkó et al. (2006) (open circles), Zhang et al. (2006) (open triangles), and the hot+warm luminosities obtained in the present work via blackbody continuum matching (solid squares; see Section 3.3 and Table 7, Column 11). The phases of the Vinkó et al. and Zhang et al. BLCs have been shifted to an explosion date of MJD $=53196.0$, the luminosities scaled to $3.13 \mathrm{Mpc}$, and the extinction to $A_{V}=0.31 \mathrm{mag}$. These BLCs are compared with the radioactive deposition power for SN 1987A as specified by Li et al. (1993) and Timmes et al. (1996). Two scaled cases are shown viz. $0.0095 M_{\odot}$ (solid line) and $0.016 M_{\odot}$ (dashed line) of ${ }^{56} \mathrm{Ni}$. We also show the total radioactive luminosity (red) for the $0.0095 M_{\odot}$ case. In addition (dotted line), there are the actual UV-augmented bolometric light curves of SN 1987A (Pun et al. 1995) scaled to the case of an initial ${ }^{56} \mathrm{Ni}$ mass of $0.0095 M_{\odot}$. The divergence between the two SN 1987A data sets at late times is ascribed to differences in the IR flux measurements between the two observatories at, respectively, CTIO (lower curve) and ESO (upper curve).

(A color version of this figure is available in the online journal.)

Vinkó et al. and Zhang et al. by $19 \%$ and $10 \%$, respectively. We scaled the Vinkó et al. (2006) BLC by a further $\times 1.1$ in order to allow approximately for the higher total extinction ( $A_{V}=0.31 \mathrm{mag}$ ) adopted by Vinkó et al. (2009) and the present work. We scaled the BLC of Zhang et al. (2006) by a further factor of 0.68 to force agreement with our adjusted version of the Vinkó et al. BLC. The need for this was due to the much larger extinction, $A_{V}=1.02 \mathrm{mag}$, adopted by Zhang et al., compared with the $A_{V}=0.31 \mathrm{mag}$ adopted in the present work. It was found that these adjustments brought our 89 days, 106 days, and 129 days blackbody total luminosities (see Section 3.3 and Table 7, Column 11) into fair coincidence with the other two BLCs (see Figure 12).

Vinkó et al. (2006) constructed their BLC by integrating observed fluxes in the $B V R I$ bands and then extrapolating linearly from the $B$ and $I$ fluxes assuming zero flux at $3400 \AA$ and $23000 \AA$. Their BLC extended to 307 days. Zhang et al. (2006) simply integrated the observed fluxes in 12 narrow bands between $4000 \AA$ and $10000 \AA$. Their BLC extended to 154 days, with five additional points to 180 days obtained by interpolation within a reduced number of bands. Thus, neither of these BLCs included unobserved excess flux beyond about $1 \mu \mathrm{m}$. However, for 89-129 days the unobserved MIR flux made up no more than $10 \%$ of the total luminosity (see Section 3.4.2). Moreover, the optical/NIR region was dominated by continuum emission at this time. Consequently, the hot+warm continuum 
Table 7

Parameters for Triple-blackbody Matches to SN 2004dj Continua

\begin{tabular}{|c|c|c|c|c|c|c|c|c|c|c|c|c|}
\hline $\begin{array}{l}\text { Epoch } \\
\text { (days) }\end{array}$ & $\begin{array}{c}v_{\text {hot }} \\
\left(\mathrm{km} \mathrm{s}^{-1}\right)\end{array}$ & $\begin{array}{l}T_{\text {hot }} \\
(\mathrm{K})\end{array}$ & $\begin{array}{c}v_{\text {warm }} \\
\left(\mathrm{km} \mathrm{s}^{-1}\right)\end{array}$ & $\begin{array}{c}R_{\mathrm{warm}} \\
\left(10^{15} \mathrm{~cm}\right)\end{array}$ & $\begin{array}{c}T_{\text {warm }} \\
(\mathrm{K})\end{array}$ & $\begin{array}{c}v_{\text {cold }} \\
\left(\mathrm{km} \mathrm{s}^{-1}\right)\end{array}$ & $\begin{array}{c}T_{\text {cold }} \\
(\mathrm{K})\end{array}$ & $\begin{array}{c}L_{\mathrm{hot}}^{\mathrm{a}} \\
\left(10^{38}\right. \\
\left.\mathrm{erg} \mathrm{s}^{-1}\right)\end{array}$ & $\begin{array}{c}L_{\mathrm{warm}}^{\mathrm{a}} \\
\left(10^{38}\right. \\
\left.\mathrm{erg} \mathrm{s}^{-1}\right)\end{array}$ & $\begin{array}{c}L_{\text {cold }}^{\mathrm{a}} \\
\left(10^{38}\right. \\
\left.\operatorname{erg~s}^{-1}\right)\end{array}$ & $\begin{array}{c}L_{\text {total }}^{\mathrm{b}} \\
\left(10^{38}\right. \\
\left.\mathrm{erg} \mathrm{s}^{-1}\right)\end{array}$ & $\begin{array}{c}L_{\mathrm{rad}} \\
\left(10^{38}\right. \\
\left.\operatorname{erg~s}^{-1}\right)\end{array}$ \\
\hline 89 & 450 & 7000 & 1750 & 1.3 & 1800 & 7000 & 200 & 2141 & 135 & 0.33 & 2276 & 592 \\
\hline 106 & 220 & 7000 & 1300 & 1.2 & 1800 & 8500 & 200 & 695 & 106 & 0.69 & 801 & 507 \\
\hline 129 & 180 & 6000 & 1250 & 1.4 & 1300 & 7500 & 200 & 372 & 39.5 & 0.80 & 412 & 411 \\
\hline 251 & 50 & 6000 & 580 & 1.3 & 1000 & 5000 & 200 & 109 & 11.3 & 1.3 & 120 & 137 \\
\hline 281 & 50 & 5300 & 470 & 1.1 & 900 & 4500 & 200 & 82.9 & 6.1 & 1.4 & 89 & 103 \\
\hline 500 & 3.8 & 10000 & 570 & 2.5 & 840 & 1800 & 200 & 19.2 & 21.5 & 0.69 & 41 & 10.7 \\
\hline 652 & (1.0) & $10000^{c}$ & 550 & 3.1 & 700 & 1400 & 200 & 2.3 & 16.4 & 0.71 & 19 & 2.1 \\
\hline 859 & $(0.16)$ & $10000^{\mathrm{c}}$ & 450 & 3.3 & 620 & 1250 & 200 & 0.10 & 11.7 & 0.98 & 12 & 0.26 \\
\hline 996 & $(0.05)$ & $10000^{c}$ & 440 & 3.8 & 530 & 800 & 200 & 0.013 & 8.1 & 0.5 & 8.1 & 0.077 \\
\hline 1207 & $(0.01)$ & $10000^{c}$ & 330 & 3.4 & 480 & 1050 & 200 & $7.8 \times 10^{-4}$ & 4.5 & 1.4 & 4.5 & 0.019 \\
\hline 1393 & $(0.0025)$ & $10000^{c}$ & 320 & 3.8 & 440 & 900 & 200 & $6.5 \times 10^{-5}$ & 4.0 & 1.3 & 4.0 & 0.009 \\
\hline
\end{tabular}

Notes.

a The continuum luminosities tend to underestimate the total SN luminosity as they do not include line emission. This is particularly the case around 200-500 days when the relative contribution of nebular line emission is at a maximum. There is less of a problem before this era when the hot continuum dominates, or afterward when the warm/cold continuum increasingly dominates.

b $L_{\text {total }}$ excludes $L_{\text {cold. }}$ This is because the cold component is due to an IS IR echo (see Section 3.4.3.1) which was predominantly powered by the peak luminosity of the SN prior to the earliest epoch of observation.

${ }^{c}$ Hot blackbody velocities and temperatures estimated by fixing the temperature at the 500 days value and extrapolating the earlier velocity evolution. In Column $13, L_{\text {rad }}$ is the radioactive deposition corresponding to the ejection of $0.0095 M_{\odot}$ of ${ }^{56} \mathrm{Ni}$, scaled from the SN $1987 \mathrm{~A}$ case specified by Li et al. (1993) and Timmes et al. (1996). The 89 days and 996 days warm and cold parameters are based on photometry only.

luminosities obtained from the present work via blackbody matching (see Section 3.3 and Table 7, Column 12) and plotted in Figure 12 (solid squares) agree well with the two adjusted BLCs. (We exclude the cold component because, as argued in Section 3.4.3.1, it is due to an IS IR echo which was predominantly powered by the peak luminosity of the $\mathrm{SN}$ prior to the earliest epoch of observation.) By 251/281 days, our hot+warm continuum luminosities make up only $\sim 60 \%$ of the adjusted Vinkó et al. BLC. This is due to the relatively strong contribution of line emission to the total luminosity during this time. Line emission luminosity was not included in our blackbody matches (see Section 3.3).

In Figure 12, the SN BLCs are compared with the radioactive deposition power in SN 1987A, as specified by Li et al. (1993) and Timmes et al. (1996). These radioactive decay light curves are scaled to, respectively, $0.0095 M_{\odot}$ (solid line) and $0.016 M_{\odot}$ (dashed line) of ${ }^{56} \mathrm{Ni}$. Also shown (red) is the total radioactive luminosity in the case of $0.0095 M_{\odot}$. We also show (dotted lines) the actual UV-augmented BLCs of SN 1987A (Pun et al. 1995) derived from observations at ESO and CTIO, scaled to an initial ${ }^{56} \mathrm{Ni}$ mass of $0.0095 M_{\odot}$. It can be seen that the $0.0095 M_{\odot}$ case provides a good match to the SN 2004dj BLC during 115-150 days, just after the end of the plateau phase. After 150 days, unlike SN 1987A, the SN 2004dj BLC begins to exceed the luminosity of the $0.0095 M_{\odot}$ case with the discrepancy growing steadily with time. Indeed, even the total radioactive luminosity of the scaled SN 1987A is exceeded by the SN 2004dj light curve, implying that an additional source of energy has appeared.

Our $0.016 M_{\odot}$ deposition plot corresponds approximately to the $0.02 M_{\odot}$ case of Vinkó et al. (2006) in their Figure 18. We agree that this case provides a fair match to the BLC during 260-310 days. Nevertheless, viewed within the context of the whole BLC, it can be seen that this "match" is actually due to an inflection section during the growth of the BLC excess relative to the true radioactive deposition. Adoption of the $0.016 M_{\odot}$ case would imply an unexplained BLC deficit during $\sim 95-250$ days. Given the phase of the event and the unexceptional progenitor mass, it is difficult to see how such a discrepancy would come about. Consequently, we argue that only during the 115-150 days phase was the BLC of SN 2004dj actually dominated by radioactive decay. Beyond this period, and as deduced also in Sections 2.2 and 2.3, an additional luminosity source emerged. We therefore reject the ${ }^{56} \mathrm{Ni}$ mass deduced by Vinkó et al. (2006).

We also reject larger ${ }^{56} \mathrm{Ni}$ masses reported by other authors. Kotak et al. (2005) used the $V$-band exponential tail method of Hamuy (2003) to derive a ${ }^{56} \mathrm{Ni}$ mass of $\sim 0.022 M_{\odot}$. This was based on the $V$ magnitude at 100 days which the subsequently more complete database shows was not quite yet on the radioactive tail (see Figure 4), thus leading to an overestimate of the ${ }^{56} \mathrm{Ni}$ mass. This method was also one of those used by Zhang et al. (2006) who obtained $0.025 \pm 0.010 M_{\odot}$ of ${ }^{56} \mathrm{Ni}$. As already indicated, their larger value was due mostly to their much earlier explosion epoch and much larger extinction, neither of which we view as likely. Chugai et al. (2005) obtained $0.020 \pm 0.006 M_{\odot}$ of ${ }^{56} \mathrm{Ni}$ based on comparison of the $V$ magnitude at 200 days with that of SN 1987A. The difficulty here is that by this epoch (as also in the Vinkó et al. 2006 case) an additional power source had appeared in SN 2004dj, biasing the derived ${ }^{56} \mathrm{Ni}$ mass to higher values. In addition, Chugai et al. (2005) used an exceptionally early explosion date, pushing their result even higher. Finally, we note that both Chugai et al. (2005) and Zhang et al. (2006) also used the $V$-light curve "steepness" method of Elmhamdi et al. (2003), which is insensitive to distance and extinction uncertainties. Chugai et al. obtained $0.013 \pm$ $0.004 M_{\odot}$, consistent with our result. Zhang et al. applied the same method to a number of wavebands, including $V$, but obtained a larger $0.020 \pm 0.002 M_{\odot}$. Their steepness parameter at just $V$ yields about $0.019 M_{\odot}$ suggesting that their use of multiple bands is not the cause of the apparent disagreement with Chugai et al. However, the difference between the Chugai et al. and Zhang et al. determinations is only at the level of $\sim 1.5 \sigma$ significance.

We conclude that, taking into account the uncertainties in fluxing, adopted distance, extinction, and explosion epoch (see 


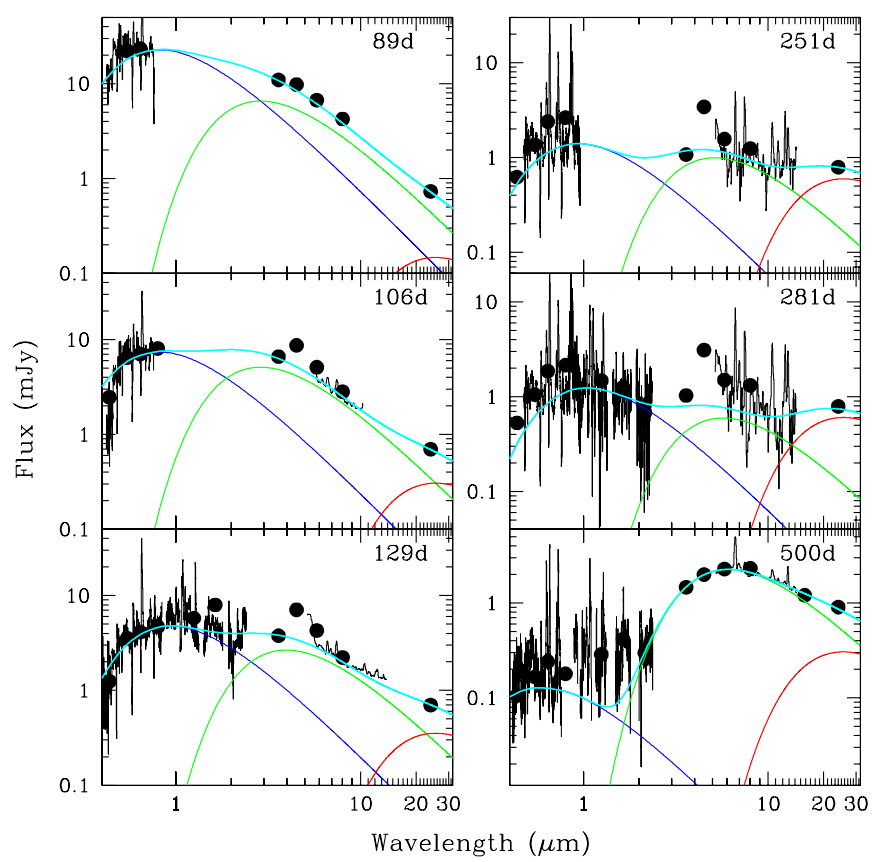

Figure 13. Comparison of blackbody continua with the optical-NIR-MIR photometric fluxes and spectra of SN 2004dj at six epochs spanning 89-500 days. The solid dots show contemporary photometry. The blue, green, and red lines show, respectively, the hot, warm, and cold blackbodies. The cyan line shows the total model spectrum.

(A color version of this figure is available in the online journal.)

Section 1.1) the mass of ${ }^{56} \mathrm{Ni}$ ejected by $\mathrm{SN} 2004 \mathrm{dj}$ was $0.0095 \pm 0.002 M_{\odot}$. We adopt this value for the rest of the paper.

\subsection{Comparison of Observed Continua with Blackbody Radiation}

Here we begin to consider the location and energy source of the SN continuum, especially longward of $2 \mu \mathrm{m}$ where thermal emission from dust would appear. To take an initially neutral standpoint on the interpretation, we compared optical, NIR and MIR spectra and photometry with blackbody continua. This provides us with the minimum radii of the emitting surfaces. The epochs were selected primarily as those for which MIR spectra were available, although the earliest such epoch, 106 days, was already during the nebular era. The earliest MIR photometry was acquired at 89 days when the SN light curve was only about half-way down the fall from the plateau to the nebular level. Given the potential interest of this epoch, we began our model comparisons at this epoch despite the lack of an MIR spectrum. In addition, to compensate for the large gap between 859 days and 1207 days we also considered the 996 days SED based on MIR photometry only. Optical photometry was taken from Vinkó et al. (2006, 2009) and Zhang et al. (2006). Details about the sources of the other data are given in Tables 1-4. Apart from 89 days and 996 days, all the optical and NIR data plus the MIR photometry were flux scaled by interpolation of the light curves to the epochs of contemporaneous MIR spectra.

The contribution of S96, represented by a $3500 \mathrm{~K}$ blackbody of radius $1.5 \times 10^{14} \mathrm{~cm}$ (see Section 3.1), was first subtracted from all the data. To model the resulting $0.4-24 \mu \mathrm{m} \mathrm{SN}$ continuum it was found to be necessary to use three blackbodies ("hot:" 5300-10000 K, "warm:" 320-1750 K, and "cold:" $\sim 200 \mathrm{~K})$. These were reddened and then matched visually to

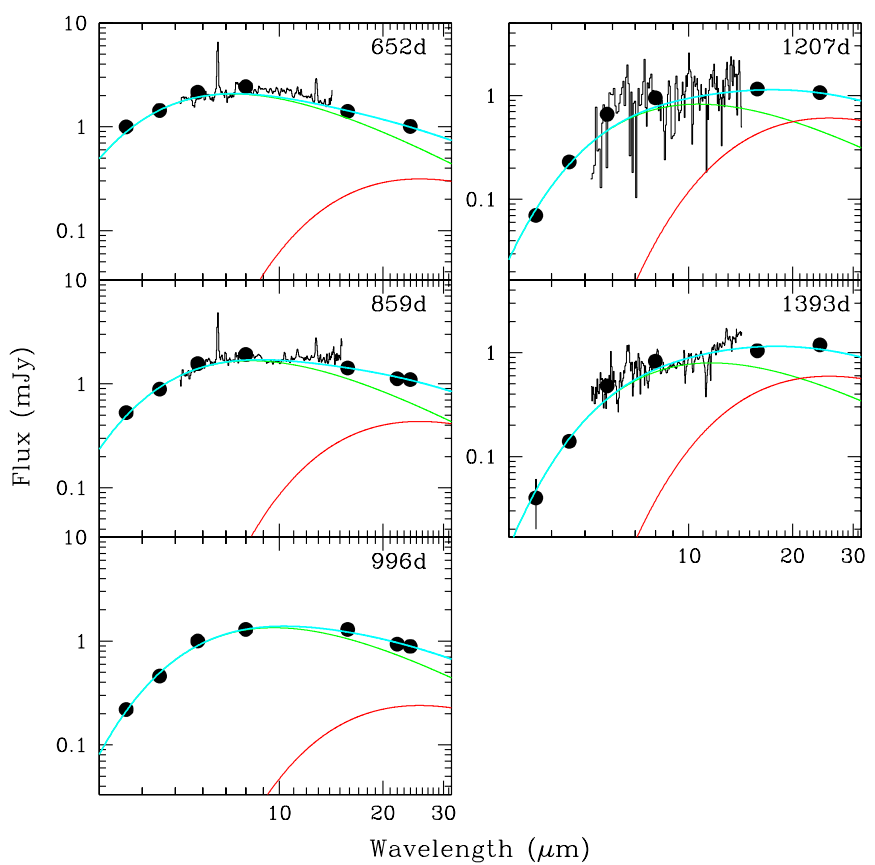

Figure 14. Comparison of blackbody continua with the MIR photometric fluxes and spectra of SN 2004dj at five epochs spanning 652-1393 days. The solid dots show contemporary photometry. The green and red lines show, respectively, the warm and cold blackbodies. The cyan line shows the total model spectrum. A hot component was also included in the matches but had a negligible effect on the warm-cold matches to the MIR continua. Consequently, and in order to show the MIR behavior in more detail, the optical/NIR region is not shown. Estimates of the hot component contributions are given in Table 7.

(A color version of this figure is available in the online journal.)

the continua. The hot blackbody was first added and adjusted to match the optical continuum. While the hot blackbody provides some information about the energy budget of the shorter wavelength part of the spectrum, the main reason for its inclusion in this study was to allow correction for its effect on the net continuum in the NIR where, up to about 500 days, it is comparable in strength to the warm component. The warm blackbody was then added and adjusted to match the $\sim 5-10 \mu \mathrm{m}$ continuum plus the long-wavelength end (2-2.4 $\mu \mathrm{m})$ of the NIR continuum. It was found that, starting at the earliest epoch, as the $\mathrm{SN}$ evolved the hot+warm blackbody flux longward of $10 \mu \mathrm{m}$ increasingly fell below that of the observations. Therefore, a cold blackbody was added and adjusted to provide the final match. For epochs where photometry but no spectra were available we used the temporally nearest spectral matches to indicate the likely position of the underlying continuum. The expansion velocities of the blackbody surfaces, $v_{\text {hot }}, v_{\text {warm }}$, and $v_{\text {cold }}$, and temperatures, $T_{\text {hot }}, T_{\text {warm }}$, and $T_{\text {cold }}$, for these matches are tabulated in Table 7 . The warm blackbody radii, $R_{\text {warm }}$, are also listed. The model matches are displayed in Figure 13 (89-500 days) and Figure 14 (652-1393 days). In Table 7, we also show the luminosities, $L_{\text {hot }}, L_{\text {warm }}$, and $L_{\text {cold }}$, of the three blackbody components together with the sum of the hot and warm components $\left(L_{\text {total }}\right)$. We exclude the cold component because, as will be argued in Section 3.4.3.1, it is due to an IS IR echo which was predominantly powered by the peak luminosity of the SN prior to the earliest epoch of observation. In the final column is listed the radioactive deposition power corresponding to the ejection of $0.0095 M_{\odot}$ of ${ }^{56} \mathrm{Ni}$, scaled from the SN 1987A case specified by Li et al. (1993) and Timmes et al. (1996).

We stress that the blackbody matches were to the underlying spectral continua where this could be reasonably judged, and 
not to the photometric points which also contained flux from line emission. Thus, the models sometimes lie below the average level of the spectra. This is particularly so for later epochs at wavelengths shortward of $2 \mu \mathrm{m}$ where the spectra are dominated by broad, blended emission lines. This tends to mask most of the underlying thermal continuum, leading to a possible overestimation of the hot continuum. Nevertheless, the blackbody luminosities tend to underestimate the total luminosity as they do not allow for the total line emission. This is particularly the case around 200-500 days when the relative contribution of nebular line emission to the total luminosity is at a maximum. There is less of a problem before this era when the hot continuum is relatively strong, or afterward when the warm/ cold continuum increasingly dominates. Also, by 652 days, the relative weakening of the hot continuum means that it has a negligible effect at wavelengths longward of $2 \mu \mathrm{m}$. In the $2-14 \mu \mathrm{m}$ region, the true continuum level is easier to judge. By 251 days and later, the total $2-14 \mu \mathrm{m}$ flux exceeds that of the continuum model by no more than $25 \%$.

The hot continuum declined monotonically and dominated the total SN continuum luminosity up to about a year postexplosion. It presumably arose from hot, optically thick ejecta gas. At 89 days most of the hot continuum was probably still driven by the shock-heated photosphere, with the remainder being due to radioactive decay (see Table 7 and Figure 12). After 500 days the hot continuum became relatively weak and of a low signal-to-noise ratio $(\mathrm{S} / \mathrm{N})$. In addition, the NIR spectral data set ended on 554 days. For subsequent epochs, the strength of the hot component was estimated by extrapolation. At 500 days the hot-blackbody match was achieved with a temperature of $10,000 \mathrm{~K}$. Between 251 days and 500 days, the blackbody velocity declined exponentially with an $e$-folding time of $\sim 130$ days and so, for epochs after 500 days the hotblackbody temperature was fixed at $10,000 \mathrm{~K}$ and the velocity obtained by extrapolation of the earlier exponential behavior (see Table 7). While this is likely to be increasingly inaccurate with time, it is unlikely to be a serious source of error in determining the warm continuum; for example, by 500 days the hot blackbody contributed barely $1 \%$ of the flux at $3.6 \mu \mathrm{m}$. Consequently, and in order to illustrate the MIR behavior in more detail, the optical/NIR region is not shown in the later plots (Figure 14).

The warm component luminosity declined monotonically to 281 days but then, unlike the hot component, increased by a factor of 3.5 by 500 days. By that time the hot+warm SN continuum luminosity exceeded that of the radioactive energy input by a factor of four and this excess continued to increase with time. The cause of the growing excess was the warm component luminosity. We also note that $R_{\text {warm }}$ is more than doubled between 281 days and 500 days, but remained roughly constant thereafter. In fact, as we show later (Sections 3.4.4.1 and 3.4.4.2), after 500 days slow shrinkage in the size of the warm emission region occurred.

The cold component is primarily defined by the $24 \mu \mathrm{m}$ point and can be fairly reproduced using a range of temperatures $(150-300 \mathrm{~K})$ and velocities. Its luminosity remained roughly constant throughout the observations. In Table 7, we show the case with the temperature fixed at $200 \mathrm{~K}$.

It is interesting that, even as early as 89 days, warm and cold blackbodies had to be included to achieve a fair match to the observed fluxes in the NIR-MIR region. This will be discussed in Sections 3.4.2 and 3.4.3.1. By 106 days, the nebular phase was just beginning and by 129 days the hot+warm luminosity was driven predominantly by radioactive decay. By 251 days and 281 days, the radioactive deposition exceeded the sum of the hot+warm blackbody luminosity by about $15 \%$. This excess probably went into powering the line emission not included in the blackbody matches. As already indicated, the nebular line emission was particularly strong at this time. Indeed, it was responsible for a $\sim 50 \%$ excess of the total bolometric luminosity relative to the radioactive deposition (Figure 12), indicating the emergence of an additional energy source, probably the reverse shock (Section 3.4.4.2). The appearance of an additional power source has already been indicated by the flattening of the optical light curves (Section 2.2). Indeed, after $\sim 150$ days the true BLC exhibited a steadily growing excess relative to the radioactive deposition (see Figure 12).

The hot and warm blackbody velocities never exceeded $1750 \mathrm{~km} \mathrm{~s}^{-1}$, indicating continuum emission consistent with an origin in the ejecta or ejecta/CSM interface. The cold component exhibits velocities between $4500 \mathrm{~km} \mathrm{~s}^{-1}$ and $8500 \mathrm{~km} \mathrm{~s}^{-1}$ during the earlier phase pointing to an origin more likely to be outside the ejecta, specifically an IR echo from pre-existing dust.

We conclude that the IR continuum comprised at least two components. The temperatures and temporal variation of these components point to thermal emission from dust whose energy source is ultimately the SN. The surge in the luminosity of the warm component by 500 days suggests the emergence of an additional source of radiation-i.e., that the warm component was driven by different energy sources at, respectively, early and late times. It also raises the possibility that distinct dust populations were responsible for the early- and late-time warm components.

\subsection{Origin of the Infrared Radiation}

We now explore the origin of the IR continuum radiation from SN 2004dj, especially the warm component. To do this, we have constructed a model continuum comprising hot gas, warm local dust, and cold IS dust. We also made use of spectral line red-wing suppression in the study of the warm dust. The continuum model was adjusted to provide visual matches to the observations.

\subsubsection{The Hot Component}

As in Section 3.3, the hot continuum component, presumably due to optically thick ejecta gas, was represented using a hot blackbody having a temperature of 5300-10,000 K. This blackbody radius and temperature were adjusted to obtain a match to the optical-NIR continuum. The warm component model (see Sections 3.4.2 and 3.4.4.2) was then added and adjusted to match the $\sim 5-10 \mu \mathrm{m}$ continua plus the longwavelength end $(2-2.4 \mu \mathrm{m})$ of the NIR continuum. As with the pure blackbody matches it was found that, at all epochs, the warm component model flux longward of $10 \mu \mathrm{m}$ increasingly fell below that of the observations. This excess is attributed to an IS IR echo (see Section 3.4.3.1).

\subsubsection{The Warm Component: Early-phase IR Excess due to a CDS}

As pointed out above, a striking result from the hot-blackbody matches is that an NIR-MIR excess (relative to the hot component flux) was present as early as 89 days post-explosion. (For brevity we henceforth refer to the NIR-MIR continuum excess 
as the "IR excess.") The MIR spectra from 106 days onward show that this was primarily due to continuum emission. We have no reason to suspect that the IR excess in the 89 days SED was not also due to continuum emission. Indeed, the CO peak at $4.5 \mu \mathrm{m}$ is noticeably suppressed at this epoch, compared with 106 days (Figure 3 ). The obvious interpretation of the IR excess is that it arose from warm dust heated by the SN. The very early appearance of this emission, when the H-recombination front had not yet reached the He-metal core, argues against an origin in newly formed ejecta dust. In addition, SN-ejecta dust-formation models (e.g., Todini \& Ferrara 2001; Nozawa et al. 2003) suggest that dust formation in a CCSN is unlikely to occur until after one year post-explosion. A second possibility of direct shock heating of pre-existing circumstellar dust is also ruled out. The SN UV flash would evaporate dust out to $(0.5-1.0) \times 10^{17} \mathrm{~cm}(0.016-0.032 \mathrm{pc}$; see Section 3.4.3.1). To reach this distance by 89 days would require a shock velocity of at least $65,000 \mathrm{~km} \mathrm{~s}^{-1}$. Yet the velocities of the $\mathrm{H} \alpha$ trough (Korcáková et al. 2005; Vinkó et al. 2006) as well as of the extreme blue-profile edge (our measurements of the spectra of Korcáková et al. 2005; Vinkó et al. 2006) during the period 25-90 days suggest that the bulk of the ejecta never exceeded velocities much more than $\sim 15,000 \mathrm{~km} \mathrm{~s}^{-1}$. Indeed, Chugai et al. (2007) adopt a velocity of $13,000 \mathrm{~km} \mathrm{~s}^{-1}$ as the boundary velocity in their treatment of SN 2004dj. A third possibility, CSM dust heating by X-rays, is also implausible. The luminosity of the warm continuum component at 89 days was $1.3 \times 10^{40} \mathrm{erg} \mathrm{s}^{-1}$ (Table 7) but the X-ray luminosity at 30 days was only about $1 \%$ of this (Pooley \& Lewin 2004). In the case of dust in a CDS, collisional heating is ruled out as the energy available in the CDS is about a factor of $10^{5}$ less than that required to account for the observed IR excess. The heat capacity of the grains is also insufficient to account for required energy.

The most likely explanation for the early-phase IR excess is an IR echo of the SN early-time luminosity from circumstellar dust. There are two possible scenarios here. In the first of these, the IR echo is from pre-existing dust in the CSM. Such early IR echoes have been suspected before in other CCSNe. Wooden et al. (1993) found an IR excess in SN 1987A as early as 260 days and possibly also at just 60 days. They hypothesized that the origin of the excess was warm, SN-heated dust in the CSM. Wooden (1997) reiterates that the cause was CSM dust "echoing the light curve." Fassia et al. (2000) reported a strong $K-L^{\prime}$ excess in the emission from the Type IIn SN 1998 S at 130 days. They attributed this to pre-existing CSM dust heated either by the SN luminosity (a conventional IR echo) or by X-rays from the CSM-shock interaction. Pozzo et al. (2004) argued in favor of the former scenario.

In the second IR echo scenario, as the fast moving ejecta collides with the CSM, a CDS forms between the forward and reverse shocks. As noted in Section 1, Chugai et al. (2007) have used the $\mathrm{H} \alpha$ spectrum to deduce the existence of a CDS in SN 2004dj. Within the CDS conditions can allow new dust to form (Pozzo et al. 2004). CDS dust has been invoked as the origin of the early-time IR excess of SN 2006jc (Smith et al. 2008; Di Carlo et al. 2008; Mattila et al. 2008). Mattila et al. (2008) showed that the IR emission was probably an IR echo from the CDS dust. IR excesses in CCSNe at later times have also been attributed to emission from newly condensed CDS dust, viz., in the Type IIn SN 1998S (Pozzo et al. 2004) and the Type IIP SN 2007od (Andrews et al. 2010).

We explored the possibility that the early IR excess was due to an IR echo of the SN luminosity from spherical distributions of either pre-existing circumstellar dust or newly formed CDS dust. Details of the model are given in Meikle et al. (2006). It fully allows for the effects of light-travel time across the dust distribution. Versions of this model have also been used in Meikle et al. (2007), Mattila et al. (2008), Botticella et al. (2009), and Kotak et al. (2009). The model assumes a spherically symmetric cloud of grains centered on the $\mathrm{SN}$, with a concentric dust-free cavity at the center. The $\mathrm{SN}$ is treated as a point source. For simplicity, a single grain radius, $a$, is adopted. For ease of computation, we assumed that the grain material was amorphous carbon where, for wavelengths longer than $2 \pi a$, the grain absorptivity/emissivity can be well approximated as being proportional to $\lambda^{-1.15}$ (Rouleau \& Martin 1991). For shorter wavelengths, an absorptivity/emissivity of unity was used. The material density is $1.85 \mathrm{~g} \mathrm{~cm}^{-3}$ (Rouleau \& Martin 1991). Free parameters are the grain size, grain number density, radial density law, and extent. The input luminosity is a parameterized description up to 550 days of the BLC shown in Figure 12, viz.,

$L_{\text {bol }}=L_{0} \exp (-t / \tau)$, where $L_{0}=57.0, \tau=1000.0$ days for $t \leqslant 0.2$ days; $L_{0}=1.70, \tau=23.4$ days for 0.2 days $<$ $t \leqslant 23.0$ days; $L_{0}=0.66, \tau=141.9$ days for 23.0 days $<$ $t \leqslant 76.4$ days; $L_{0}=50.7, \tau=15.9$ days for 76.4 days $<t \leqslant$ 112.0 days; $L_{0}=0.079, \tau=174.8$ days for 112.0 days $<t \leqslant$ 550.0 days; and $L_{0}=0$ for $t>550.0$ days.

$L_{0}$ is in units of $10^{42} \mathrm{erg} \mathrm{s}^{-1}$. The brief but highly luminous first term represents the energy in the UV flash. The second term, which covers the pre-discovery phase, was estimated by using the BLC of SN 1999em (Elmhamdi et al. 2003), adjusted so that the epoch and luminosity of the beginning of its plateau phase coincided approximately with the earliest observed point on the SN 2004dj BLC.

For the third, fourth, and fifth terms, the adjusted BLCs of Vinkó et al. (2006) and Zhang et al. (2006) were used (see Section 3.2). As already pointed out, these BLCs did not include excess flux beyond about $1 \mu \mathrm{m}$, nor any flux beyond $2.3 \mu \mathrm{m}$; virtually all of the warm and cold components were excluded. Thus, use of the above parameterized description avoids "double-counting" of a putative CSM/CDS IR echo. Indeed, the BLC description slightly underestimates the true SN luminosity input as it excludes all IR emission longward of $2.3 \mu \mathrm{m}$, as well as all UV emission shortward of $0.34 \mu \mathrm{m}$. Consequently, the input luminosity was scaled by a factor of about 1.1 to allow for unobserved UV and IR fluxes.

For the case of pre-existing CSM dust, the model was adjusted to reproduce the IR-excess SEDs for the three earliest epochs (89, 196, and 129 days). The outer limit of the circumstellar dust was initially set at 10 times that of the cavity radius, and an $r^{-2}$ (steady wind) density profile was assumed. However, it was impossible to reproduce the quite rapid temporal decline of the IR excess without raising the temperature of the hottest grains to above their evaporation temperature. While a better match to the SED shape and evolution was obtained by setting the density profile to steeper than $r^{-4}$, the best match was achieved with a discrete, thin shell. We therefore adopted this configuration, setting the shell thickness at $\times 0.1$ the cavity radius.

We investigated a range of cavity radii. For pre-existing CSM dust the minimum size of the concentric dust-free cavity is fixed by the extent to which the dust was evaporated by the initial UV flash from the SN. In this scenario, while the BLC dominates the heating of the surviving dust, the size of the dustfree cavity is determined by the luminosity peak of the UV flash, with $r_{\text {evap }} \propto L_{\text {peak }}^{0.5}$ (Dwek 1983). For a Type IIP SN, the flash luminosity is estimated to peak at about $10^{45} \mathrm{erg} \mathrm{s}^{-1}$ (Klein \& 
Chevalier 1978; Tominaga et al. 2009) although it has never been observed directly. A similar peak luminosity is estimated for the Type IIpec SN 1987A (Ensman \& Burrows 1992). Dwek (1983) provides an approximate estimate of the flash-evaporated cavity size for a Type II SN. More recently, in a detailed study Fischera et al. (2002) determined that for SN 1987A the UV flash would have totally evaporated graphite CSM dust out to a radius of $(0.5-0.9) \times 10^{17} \mathrm{~cm}(0.016-0.029 \mathrm{pc})$. We therefore adopt the Fischera et al. estimates and apply them to the case of SN 2004dj. In any case, matches to the data are fairly insensitive to the cavity radii. Fair matches to the early-time IR-excess SEDs were obtainable with cavity radii $(0.5-1.3) \times 10^{17} \mathrm{~cm}$ $(0.016-0.042 \mathrm{pc})$ and corresponding grain radii of $0.1-0.04 \mu \mathrm{m}$. For cavity radii exceeding $\sim 0.05 \mathrm{pc}$, the model continuum slopes were inconsistent with the observations.

As an example of a pre-existing CSM dust model match we have cavity radius: $0.7 \times 10^{17} \mathrm{~cm}(0.023 \mathrm{pc})$; shell thickness: $0.7 \times 10^{16} \mathrm{~cm}(0.0023 \mathrm{pc})$; grain size: $0.07 \mu \mathrm{m}$; grain number density: $6.0 \times 10^{-9} \mathrm{~cm}^{-3}$; and total grain mass: $0.38 \times 10^{-5} M_{\odot}$.

The optical depth through the shell in the optical band is 0.006 , which is easily encompassed within the observed total extinction of $A_{V}=0.3 \mathrm{mag}$. For a dust/gas mass ratio of 0.005 (see Section 3.4.3.1), the dust mass corresponds to a total shell mass of $0.76 \times 10^{-3} M_{\odot}$. For the adopted shell thickness and a typical RSG wind velocity of $20 \mathrm{~km} \mathrm{~s}^{-1}$, this mass would be produced by a mass loss event about 1100 years ago, lasting for 110 years, with a mass loss rate of $7 \times 10^{-6} M_{\odot} \mathrm{yr}^{-1}$. However, while this mass loss and loss rate are plausible for RSGs, such discrete events are not thought to occur in this type of star. This prompts us to seek a more natural explanation for the thin dust shell. An obvious candidate is the CDS inferred by Chugai et al. (2007).

For the CDS scenario, the size of the cavity is essentially the radius of the CDS. From Chugai et al. (2007), the CDS radius is given by

$$
r_{\mathrm{CDS}}=5.2 \times 10^{15} \times((t+11.5) / 64.0)^{6 / 7} \mathrm{~cm},
$$

where $t$ (in days) is with respect to our explosion epoch. Thus, at the earliest of our epochs, 89 days, the CDS radius was just $7.7 \times 10^{15} \mathrm{~cm}$, or about one-tenth of the pre-existing CSM dust cavity. This is only three light days implying that light-travel time effects are small. Nevertheless, for convenience and ease of comparison with the pre-existing dust case, we applied the IR echo model to the CDS case. Although the Chugai et al. study terminates at just 99 days, we assume that the CDS radius continues to increase as described in Equation (1) until at least 500 days, by which time its contribution to the IR excess is small.

We compared the CDS case of the IR echo model with the observations, with the dust lying in a thin shell of radius $r_{\mathrm{CDS}}$ as given above. We found that setting the dust mass at a constant value produced a poor match to the observations. With a match at 106 days, the model at 89 days yielded a continuum which matched the observed IR excess at $8 \mu \mathrm{m}$ but exceeded the IR excess by nearly $\times 2$ at $3.6 \mu \mathrm{m}$. On the other hand, at 129 days, while the model continuum slope was similar to that of the observed IR excess, it significantly underproduced it with the deficit being as large as $\sim 35 \%$ at $8 \mu \mathrm{m}$. We propose that this problem is due to the unjustified assumption of a fixed CDS dust mass. CDS dust could not form until the SN flux at the CDS had faded sufficiently for proto-dust material to cool below the condensation temperature. This occurred at about
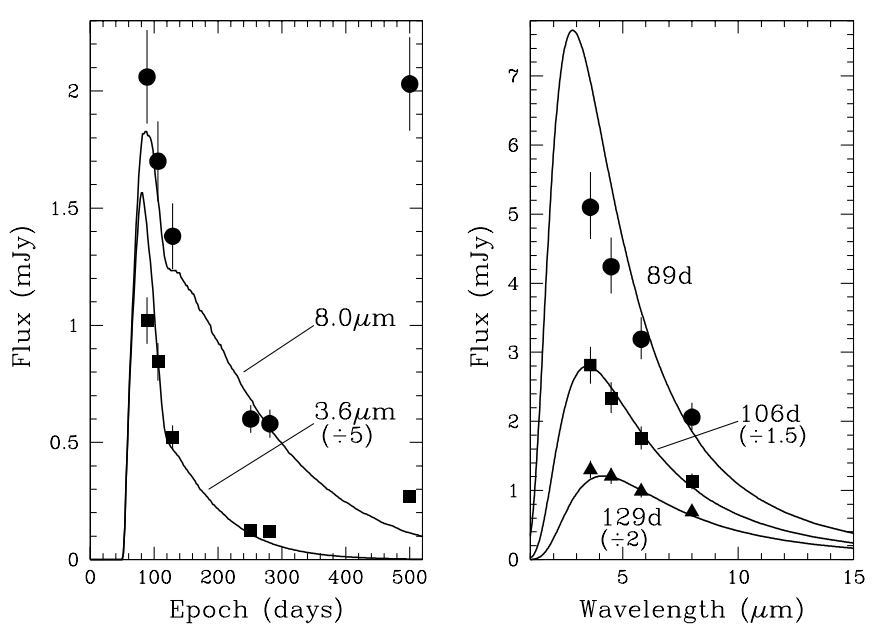

Figure 15. CDS IR echo model compared with the $3.6 \mu \mathrm{m}$ and $8.0 \mu \mathrm{m}$ MIR excess light curves of SN 2004dj (LH panel), and the 89, 106, and 129 days MIR excess SEDs (RH panel). The grain growth began at 50 days with a growth timescale also of 50 days. Amorphous carbon grains were adopted of radius $0.2 \mu \mathrm{m}$. The final dust mass is $0.33 \times 10^{-5} M_{\odot}$.

50 days, assuming amorphous carbon dust. Therefore, following Mattila et al. (2008) we allowed the CDS dust mass to grow as $M_{d}=M_{0}\left(1-\exp \left(-\left(t-t_{0}\right) / t_{d}\right)\right)$, where $t$ is time, $t_{0}$ is the time at which dust condensation began (set at 50 days), $t_{d}$ is the characteristic grain growth timescale, and $M_{0}$ is the asymptotically approached final mass. (We note that Mattila et al. also deduced an epoch of 50 days for the start of the CDS dust condensation in SN 2006jc.) No attempt was made to simulate the growth of individual grains which were assumed to appear instantaneously at their final size. Owing to the lighttravel time differences across the CDS, the grain condensation is seen to commence during the epochs $\left(t_{0}-\left(r_{\mathrm{CDS}} / c\right)\right)$ to $\left(t_{0}+\left(r_{\mathrm{CDS}} / c\right)\right)$ days. Yet, even as late as 500 days, $\left(r_{\mathrm{CDS}} / c\right)$ was only about 12 light days and so this effect was ignored.

In Figure 15, we compare the CDS model light curves and spectra with, respectively, the observed MIR excess fluxes at $3.6 \mu \mathrm{m}$ and $8.0 \mu \mathrm{m}$ (left-hand, hereafter LH panel), and with the 89, 106, and 129 days MIR excess SEDs (right-hand, hereafter RH panel). The free parameters for the CDS case were the dust-mass scaler, the grain radius, and the grain growth timescale. Satisfactory reproduction of the IR excess was achieved for all five epochs spanning 89-281 days with $t_{d}=50$ days, a grain radius of $0.2 \mu \mathrm{m}$, and a final dust mass of $M_{0}=0.33 \times 10^{-5} M_{\odot}$, similar to the dust mass derived in the pre-existing CSM case above. The CDS mass is $3.2 \times 10^{-4} M_{\odot}$ (Chugai et al. 2007) indicating a plausible final dust/gas mass ratio of 0.01 . The hottest dust ranged from $1330 \mathrm{~K}$ at 89 days declining to about $640 \mathrm{~K}$ at 281 days. The optical depth in the UV/optical range was 0.077 at 89 days falling to 0.011 by 500 days-i.e., consistent with the observed total extinction of $A_{V}=0.3 \mathrm{mag}$. By 500 days the MIR excess exceeds that of the model by a significant factor (especially at longer wavelengths; Figure 15) implying the appearance of an additional energy source. The CDS contribution to the SN continua for 89-500 days is plotted in Figure 18. The luminosity contribution of the CDS IR echo, $L_{\mathrm{CDS}}$, is listed in Table 9, Column 3. For epochs after 500 days the CDS component was negligible and so was ignored.

We conclude that the early-time IR excess was probably due primarily to an IR echo from newly formed dust lying within the CDS. The rapid decline of the IR excess flux, especially between 89 days and 106 days, is due largely to the ongoing fall from 
the BLC plateau, tempered by the growth of CDS dust during this period. Owing to the small size of the CDS, light-travel time effects are small. In contrast, in the pre-existing CSM dust scenario, the rapid decline of the input luminosity is tempered by the larger size of the dusty shell which produces significant light-travel time effects, smoothing the observed IR excess light curve over longer timescales. Nevertheless, given the natural explanation by the CDS model of the required thin shell dust distribution, in the completion of the analysis below we use the CDS scenario.

\subsubsection{The Cold Component}

3.4.3.1. An Interstellar Echo. The cold component is defined primarily by the $24 \mu \mathrm{m}$ data and can be fairly reproduced using a range of temperatures (150-300 K) and velocities. As described above (Section 3.3), the luminosity of the cold component remained roughly constant throughout the observations. In addition, the cold blackbody velocities were high, arguing against an origin in ejecta dust. An obvious alternative is an IS IR echo. The possibility of detecting the reflection of SN optical light from IS grains was first suggested by van den Bergh (1965). In the IR a potentially much more important phenomenon is the absorption and re-radiation by the grains of the SN BLC energy (the "IR echo"). The possibility of detecting an IS IR echo from an SN was first proposed by Bode \& Evans (1980). ${ }^{17}$ The occurrence of cold dust IS IR echoes should be relatively common for SNe occurring in dusty, late-type galaxies. The SEDs of such echoes tend to peak in the $20-100 \mu \mathrm{m}$ region, allowing echo detection in nearby galaxies by Spitzer during its cold mission. Spitzerbased evidence of this phenomenon in the Milky Way Galaxy has been presented for the Cassiopeia A SN (Krause et al. 2005; Kim et al. 2008; Dwek \& Arendt 2008). Meikle et al. (2007) showed that an IS IR echo provided a natural explanation for the strength and decline of the $24 \mu \mathrm{m}$ flux between 670-681 days and 1264 days in SN 2003gd in the SA(s)c galaxy NGC 628 (M74). Kotak et al. (2009) showed that the cold component of the SN 2004et SED was most likely due to an IS IR echo in the SAB(rs)cd host galaxy NGC 6946. The host galaxy of SN 2004dj, NGC 2403, is of type SAB(s)cd and so there is a good likelihood of a similar IS IR echo occurring. Therefore, we included an IS IR echo component in our modeling of the SED.

Our IS IR echo model is the same as that used for the earlytime CDS IR echo (Section 3.4.2). Only the dust distribution and grain radii are different. We ignore the CDS dust emission derived in Section 3.4.2 since this is already invoked in the CDS echo model and included in the continuum modeling up to 500 days. We recognize that a more extended, lower density CSM may also have existed. However, we found that the addition of yet another model component was unnecessary to provide plausible matches to the continua and so for simplicity the possible effects of an extended CSM were ignored.

As described in Section 3.4.2, pre-existing dust surrounding the $\mathrm{SN}$ would have been evaporated by the $\mathrm{SN}$ flash out to a distance of about $0.025 \mathrm{pc}$. At sufficiently late epochs, enlargement of this cavity can be produced by the forward shock. Assuming that the shock velocity is comparable to the highest ejecta velocity viz. $\sim 15,000 \mathrm{~km} \mathrm{~s}^{-1}$ (see Section 3.4.2), and that there was no deceleration, the edge of the UV-flash-determined cavity would be reached after about 600 days. After this time, the $\mathrm{SN}$ shock would evaporate the dust and enlarge the dust-free

\footnotetext{
17 Wright (1980) also considered an IR echo from an SN but only in the more
} restricted case of an explosion within a molecular cloud. cavity. Therefore, for epochs earlier than 600 days we fixed the inner limit of the IS dust at $0.025 \mathrm{pc}$. For later epochs, the inner limit of the IS dust increases from $0.028 \mathrm{pc}$ (34 light days) at 662 days to $0.058 \mathrm{pc}$ (70 light days) by 1393 days. It might be objected that this ignores the possibility of shock deceleration. However, the IR echo contribution which the shock-evaporated dust would otherwise have made, for 600 days $>t>1393$ days, to the cold component is negligible since the cavity radius was never more than 70 light days, i.e., during this late period the SN peak luminosity would have long since passed by the dust near the cavity.

The outer limit of the IS dust is much less certain. Bendo et al. (2010) have used Spitzer imaging of NGC 2403 at $70 \mu \mathrm{m}$ and $160 \mu \mathrm{m}$ to map the dust column density via its thermal emission. They also used the far-IR images in conjunction with H I observations to map the gas/dust ratio. The objection to using such measurements in the present work is that they only provide total densities through the disk (or to the mid-plane assuming symmetry), and not directly to SN 2004dj. While it was argued above that SN 2004dj actually lies toward the front of S96, we are still faced with the uncertainty of the depth of S96 in the galaxy plane. Our approach, therefore, is to assume a spherically symmetric dust distribution centered on the $\mathrm{SN}$, with an outer limit of $100 \mathrm{pc}$. While not appropriate in principle for the outer limits of the dust in the galactic disk, for the early era being considered spherical symmetry provides a good approximation; at this epoch, the echo ellipsoid is extremely elongated and so the region of the spherical model outer surface intercepted by the ellipsoid is small. The plane of NGC 2403 appears to be tilted at an inclination of about $55^{\circ}$ roughly doubling the faceon column density, and so the adopted outer limit is equivalent to a $\sim 60$ pc scale height for the IS dust with the host galaxy face-on.

The free parameters were (1) the grain size, which influenced the dust temperatures and (2) the grain number density, which determined the luminosity. These parameters were adjusted in conjunction with the warm dust models (see Sections 3.4.2 and 3.4.4.2) to provide a match to the long-wave excess. It was found that satisfactory matches at all epochs were obtained with a grain radius of $0.1 \mu \mathrm{m}$ and a dust number density of $2.6 \times 10^{-13} \mathrm{~cm}^{-3}$ (i.e., an IS gas number density of $0.24 \mathrm{~cm}^{-3}$ and a dust/gas mass ratio of 0.005; Bendo et al. 2010.) This is comparable to the typical value of $5 \times 10^{-13} \mathrm{~cm}^{-3}$ for the Milky Way (Allen 1973) and $7.0 \times 10^{-13} \mathrm{~cm}^{-3}$ obtained by Kotak et al. (2009) for NGC 6946. In the wavelength region $(\lambda>15 \mu \mathrm{m})$ where the cold component makes a significant contribution $(>20 \%)$ to the total flux, the IS IR echo model $(15-150 \mu \mathrm{m})$ maintained a nearconstant luminosity of $\sim 2.0 \times 10^{38} \mathrm{erg} \mathrm{s}^{-1}$ between 89 days and 1393 days.

The Bendo et al. dust column density map of NGC 2403 indicates $0.05 M_{\odot} \mathrm{pc}^{-2}$ at the position of SN 2004dj. From the above dust number density from the echo model and integrated over $100 \mathrm{pc}$ we obtain just $0.003 M_{\odot} \mathrm{pc}^{-2}$. The absorption opacities used by Bendo et al. are consistent, to within a factor of $\sim 2$, with the opacity law used in the present work. Thus, we have a $\sim \times 8$ discrepancy in column density between Bendo et al. and the present work. Part of this discrepancy may be that the adopted dust outer limit is too small. The derived IS dust density is fairly insensitive to the extent of the outer limit. For example, with a $75 \%$ increase in the outer limit the model match to the long-wave continuum is retained with a reduction of just $\sim 10 \%$ in the IS dust density. Thus, at least some of the discrepancy could be removed by simply increasing the dust outer limit. 
Table 8

Hot Blackbody, Free-Free/Free-Bound, and Warm Dust Disk $(\delta=0)$ Parameters for Matches to SN 2004dj SEDs

\begin{tabular}{|c|c|c|c|c|c|c|c|c|c|c|c|}
\hline $\begin{array}{l}\text { Epoch } \\
\text { (days) }\end{array}$ & $\begin{array}{c}v_{\text {hotbb }} \\
\left(\mathrm{km} \mathrm{s}^{-1}\right)\end{array}$ & $\begin{array}{l}T_{\text {hot }} \\
(\mathrm{K}) \\
\end{array}$ & $\begin{array}{c}v_{\mathrm{fb}-\mathrm{ff}} \\
\left(\mathrm{km} \mathrm{s}^{-1}\right)\end{array}$ & $\begin{array}{c}T_{\mathrm{fb}-\mathrm{ff}} \\
(\mathrm{K})\end{array}$ & $\begin{array}{c}n_{\mathrm{e}(\mathrm{fb}-\mathrm{ff})} \\
\left(10^{6} \mathrm{~cm}^{-3}\right) \\
\end{array}$ & $\begin{array}{c}r_{\mathrm{dmax}}^{\mathrm{a}} \\
\left(10^{15} \mathrm{~cm}\right) \\
\end{array}$ & $\begin{array}{l}T_{\mathrm{d}}^{\mathrm{a}} \\
(\mathrm{K}) \\
\end{array}$ & $\tau_{0.55 \mu \mathrm{m}}$ & $\tau_{10 \mu \mathrm{m}}$ & $\tau_{24 \mu \mathrm{m}}$ & $\begin{array}{c}\begin{array}{c}M_{\text {dust }} \\
\left(10^{-4} M_{\odot}\right)\end{array} \\
\end{array}$ \\
\hline 89 & 450 & 7000 & 3420 & $(6000)$ & 100 & $\ldots$ & $\ldots$ & $\ldots$ & $\ldots$ & $\ldots$ & $\ldots$ \\
\hline 106 & 275 & 6000 & 3420 & $(6000)$ & 86 & $\ldots$ & $\ldots$ & $\ldots$ & $\ldots$ & $\ldots$ & $\ldots$ \\
\hline 129 & 180 & 6000 & 3420 & $(6000)$ & 56 & $\ldots$ & $\ldots$ & $\ldots$ & $\ldots$ & $\ldots$ & $\ldots$ \\
\hline 251 & 50 & 6000 & 3080 & 5000 & 36 & $\ldots$ & $\ldots$ & $\ldots$ & $\ldots$ & $\ldots$ & $\ldots$ \\
\hline 281 & 50 & 5300 & 2980 & 5000 & 24 & $\ldots$ & $\ldots$ & $\ldots$ & $\ldots$ & $\ldots$ & $\ldots$ \\
\hline 500 & 9.0 & 6000 & 2470 & 5000 & 1.8 & $5.2(5)$ & $650(15)$ & 9.6 & $0.45(5)$ & 0.16 & $0.22(2)$ \\
\hline 652 & (1.0) & (10000) & 2090 & 5000 & 0.6 & $4.9(5)$ & $610(15)$ & 15.3 & $0.73(10)$ & 0.26 & $0.32(5)$ \\
\hline 859 & (0.16) & (10000) & 1590 & 5000 & 0.25 & $4.6(5)$ & $570(15)$ & 17.4 & $0.82(10)$ & 0.29 & $0.33(5)$ \\
\hline 996 & $(0.05)$ & $(10000)$ & 1400 & 2500 & 0.14 & $4(1)$ & $520(30)$ & 36.7 & $1.7(5)$ & 0.62 & $0.5(1)$ \\
\hline 1207 & $(0.01)$ & (10000) & 1400 & 2500 & 0.05 & $3.8(6)$ & $460(30)$ & $>80$ & $>4$ & $>1.5$ & $>1.0$ \\
\hline 1393 & $(0.0025)$ & (10000) & 1400 & 2000 & 0.024 & $3.8(6)$ & $430(20)$ & $>120$ & $>6$ & $>2$ & $>1.5$ \\
\hline
\end{tabular}

Note.

${ }^{a} r_{\mathrm{dmax}}$ and $T_{\mathrm{d}}$ are, respectively, the radius and temperature of the warm, dusty disk model. Uncertainties in the last one or two figures are shown in brackets. Likewise for the estimated disk dust mass, $M_{\text {dust }}$ (last column). An example of uncertainties in the optical depths is shown in Column 10 for $10 \mu m$. For the hot blackbody at 652 days and later, the temperature was fixed at 10,000 K and the velocity obtained by extrapolation, and so these parameters are shown in brackets. For the fb-ff modeling, the hydrogen temperature and electron density (Columns 5 and 6) for 251-1393 days were derived from the observed hydrogen velocities for SN 2004dj (Column 4) in conjunction with the late-time SN 1987A study of Kozma \& Fransson (1998; see Section 3.4.3.2). For 89-129 days, the hydrogen temperature is shown in brackets as it is a rough estimate (see Section 3.4.3.2). Columns 9-11 give the optical depths perpendicularly through the dust disk at the wavelengths indicated. The matches also included a CDS IR echo at epochs 89-500 days and an IS IR echo at all epochs (see Sections 3.4.2 and 3.4.3.1).

An explanation for the remaining discrepancy is that the $\mathrm{SN}$ and S96 actually lie significantly above the mid-plane of NGC 2403. This is confirmed as follows. The optical depth to UV/optical photons yielded by the model is about 0.026 . We can use this to estimate independently the host extinction to SN 2004dj. The optical depth translates to an absorption-only extinction of 0.028 mag. Assuming an albedo of about 0.4 (Draine 2003), we obtain a total host extinction of $0.071 \mathrm{mag}$. This is reasonably consistent with the host-only value of $A_{V}=0.081 \mathrm{mag}$ $(R=3.1)$ obtained via the Na I D observations of Guenther $\&$ Klose (2004). A similar result is obtainable from our study of SN 2004et (Kotak et al. 2009). For SN 2004et, the IS IR echo model yields an absorption-only extinction of $0.081 \mathrm{mag}$ implying a total host extinction of about $A_{V}=0.20$ mag. Highresolution spectra of $\mathrm{Na}$ I D lines to SN 2004et gave a total (host plus Galaxy) $E(B-V)=0.41 \mathrm{mag}$ (Zwitter et al. 2004) or $A_{V}=1.27 \mathrm{mag}\left(R_{V}=3.1\right)$. Subtracting the estimated Galactic contribution of $A_{V}=1.06 \mathrm{mag}$ (Schlegel et al. 1998; Misra et al. 2007) yields a host-only value of $A_{V}=0.21 \mathrm{mag}$, in excellent agreement with the IS IR echo-based value.

One possible objection to the IS IR echo interpretation of the cold component of SN 2004dj is that the steady component of the flux around $24 \mu \mathrm{m}$ could be due to cool IS dust in S96, insufficiently corrected for by the $3500 \mathrm{~K}$ blackbody extrapolation (Section 3.1). We regard this as unlikely. As already indicated, Maíz-Apellániz et al. (2004) argue that the parent molecular cloud of S96 should have been dispersed by stellar winds and SN explosions. In addition, we have found that (1) a single set of IS IR echo parameters provided a fair match throughout the 89-1393 days covered and (2) the derived dust column density is comparable to that obtained from IS Na I D spectroscopy.

A second possible objection to the IS IR echo interpretation is that the cold component is actually due to free-free (ff) emission. This is discussed and dismissed in Section 3.4.3.2.

We conclude that the cold component of SN 2004dj was due to an IS IR echo. As originally suggested by Bode \& Evans (1980), the study of IS IR echoes from SNe can provide an independent method of measuring the IS extinction in galaxies.
3.4.3.2. Free-Free and Free-Bound Radiation. In their MIR study of SN 1987A, Wooden et al. (1993) suggest that during the 60-415 days period, a significant proportion of the MIR flux longward of $\sim 20 \mu \mathrm{m}$ was due to $\mathrm{ff}$ emission, with $\mathrm{ff}$ and free-bound $(\mathrm{fb})$ emission also contributing at shorter wavelengths. For SN 2004dj, we have looked at the possible contribution of $\mathrm{fb}-\mathrm{ff}$ radiation. It is particularly important to consider ff emission as this rises in flux toward longer wavelengths, thus potentially reducing or even dismissing an IS IR echo contribution. For consistency in this analysis, fb emission must also be included. For the wavelengths covered this is strongest in the optical/NIR region. Free-bound radiation has a strong "sawtooth" structure and the extent to which this structure is undetectable in the optical/IR continuum places a limit on its strength. For a particular temperature this, in turn, constrains the strength of the ff emission $\left(F_{\mathrm{ff}} / F_{\mathrm{fb}} \propto \sqrt{T} / \nu^{3}\right)$.

The $\mathrm{fb}-\mathrm{ff}$ radiation was assumed to arise in a hydrogen envelope centered on the SN. Kozma \& Fransson (1998) modeled the time-dependent behavior of the temperature and ionization of SN 1987A, including the hydrogen envelope, for epochs after 200 days. Therefore, we made use of their study to model the ff and fb emission from SN 2004dj at epoch 251 days and later. We adopted a hydrogen envelope of a few solar masses and used their density profile:

$$
\rho=9.1 \times 10^{-16}(t /(500 \text { days }))^{-3}\left(v /\left(2000 \mathrm{~km} \mathrm{~s}^{-1}\right)^{-2} \mathrm{~g} \mathrm{~cm}^{-3}\right. \text {. }
$$

By 251 days, the highest velocity of observable hydrogen in SN 2004dj was no more than $\sim 3100 \mathrm{~km} \mathrm{~s}^{-1}$ (Table 8). By 925 days the maximum $\mathrm{H}$ velocity observed in SN 2004dj was no more than $1400 \mathrm{~km} \mathrm{~s}^{-1}$. There were no optical or NIR spectra covering the final three MIR epochs. We therefore assumed that the maximum hydrogen velocity at these late epochs remained at $1400 \mathrm{~km} \mathrm{~s}^{-1}$. The hydrogen inner limit is less certain; a value of $1000 \mathrm{~km} \mathrm{~s}^{-1}$ was adopted for all epochs. In any event, the fb-ff luminosity from 500 days onward was completely negligible for any plausible hydrogen velocity limits (see below).

We estimated the fb-ff emission from the hydrogen within the velocity limits. This was done using the escape probability 
Table 9

Hot Blackbody, fb+ff, and Warm $(\delta=0)$ Dust-disk Luminosities Compared with Radioactive Input (Fixed Disk Radius)

\begin{tabular}{lcccccc}
\hline \hline $\begin{array}{l}\text { Epoch } \\
\text { (days) }\end{array}$ & $\begin{array}{c}L_{\mathrm{hot}} \\
\left(10^{38}\right. \\
\left.\mathrm{erg} \mathrm{s}^{-1}\right)\end{array}$ & $\begin{array}{c}L_{\mathrm{CDS}} \\
\left(10^{38}\right. \\
\left.\mathrm{erg} \mathrm{s}^{-1}\right)\end{array}$ & $\begin{array}{c}L_{\mathrm{ff}+\mathrm{fb}} \\
\left(10^{38}\right. \\
\left.\mathrm{erg} \mathrm{s}^{-1}\right)\end{array}$ & $\begin{array}{c}L_{\mathrm{IDDM}}^{\mathrm{a}} \\
\left(10^{38}\right. \\
\left.\mathrm{erg} \mathrm{s}^{-1}\right)\end{array}$ & $\begin{array}{c}L_{\text {total }} \\
\left(10^{38}\right. \\
\left.\mathrm{erg} \mathrm{s}^{-1}\right)\end{array}$ & $\begin{array}{c}L_{\mathrm{rad}} \\
\left(10^{38}\right. \\
\left.\mathrm{erg} \mathrm{s}^{-1}\right)\end{array}$ \\
\hline 89 & 2050 & 128 & 8.0 & $\ldots$ & 2186 & 592 \\
106 & 586 & 55.7 & 10.0 & $\ldots$ & 652 & 507 \\
129 & 372 & 26.8 & 7.7 & $\ldots$ & 407 & 411 \\
251 & 109 & 6.6 & 3.3 & $\ldots$ & 119 & 137 \\
281 & 82.9 & 4.8 & 4.2 & $\ldots$ & 91.9 & 103 \\
500 & 14.0 & 0.6 & 0.20 & 12.4 & 27.2 & 10.7 \\
652 & $(2.3)$ & $\ldots$ & 0.03 & 9.9 & 12.2 & 2.1 \\
859 & $(0.10)$ & $\ldots$ & 0.005 & 6.9 & 7.0 & 0.26 \\
996 & $(0.032)$ & $\ldots$ & 0.0025 & 3.9 & 3.9 & 0.077 \\
1207 & $\left(7.7 \times 10^{-4}\right)$ & $\ldots$ & 0.0006 & 2.3 & 2.3 & 0.019 \\
1393 & $\left(6.5 \times 10^{-5}\right)$ & $\ldots$ & 0.0002 & 1.8 & 1.8 & 0.009 \\
\hline
\end{tabular}

Notes. For the hot blackbody (Column 2) at 652 days and later, the temperature was fixed at $10,000 \mathrm{~K}$ and the velocity obtained by extrapolation, and so the luminosities derived from these parameters are shown in brackets. The post652 days contribution of the hot blackbody to the total luminosity is negligible. In Column $7, L_{\mathrm{rad}}$ is the radioactive deposition corresponding to the ejection of $0.0095 M_{\odot}$ of ${ }^{56} \mathrm{Ni}$, scaled from the SN 1987A case described by Li et al. (1993) and Timmes et al. (1996). No IS IR echo luminosities are shown since these were predominantly powered by the peak luminosity of the SN prior to the earliest epoch of observation. In the wavelength region $(\lambda>15 \mu \mathrm{m})$ where the cold component makes a significant contribution $(>20 \%)$ to the total flux, the IS IR echo model described in the text maintained a near-constant luminosity $(15-150 \mu \mathrm{m})$ of $\sim 2.0 \times 10^{38} \mathrm{erg} \mathrm{s}^{-1}$ between 89 days and 1393 days. ${ }^{a} L_{\text {IDDM }}$ is the luminosity of the warm, dusty disk model.

formalism (Osterbrock 1989), although in practice this was unnecessary as the matches to the data always showed that the hydrogen was very optically thin. The mass of the fb-ffemitting hydrogen fell from $\sim 4 M_{\odot}$ at 251 days to $0.75 M_{\odot}$ at 996, 1207, and 1393 days. Using the modest extrapolation of Figure 7 in Kozma \& Fransson (1998) to the velocities observed in SN 2004dj, we deduced that the hydrogen at the observed velocity limits stayed at a temperature of $T \sim 5000 \mathrm{~K}$ up to 859 days and then declined to $\sim 2000 \mathrm{~K}$ by 1393 days. The fractional ionization, $\chi_{e}$, was obtained from Figure 9 of Kozma \& Fransson by interpolation. This indicated $\chi_{e} \sim 0.02$ at 251 days, falling to $\chi_{e} \sim 0.0005$ by 1393 days. At each epoch, the $T$ and $\chi_{e}$ values were assumed to apply to all the hydrogen within the velocity limits. The Kozma \& Fransson study did not extend to epochs as early as the earliest MIR observations $(89,106$, and 129 days) of SN 2004dj. We therefore adopted $6000 \mathrm{~K}$ as a plausible temperature for these epochs and allowed the ionization to take the largest value consistent with the overall match to the observed continuum. Plausible values were obtained, viz. $\chi_{e}=0.003$ at 89 days rising to $\chi_{e}=0.005$ at 129 days. We used Gaunt factors tabulated by Hummer (1988). For the fb radiation, we used the continuum recombination coefficients of Ercolano \& Storey (2006).

The hydrogen maximum velocity, temperature, and free electron density for each epoch are listed in Table 8. The fb-ff luminosities are listed in Table 9, Column 4. It can be seen that by 500 days the fb-ff contribution to the total luminosity is small, becoming increasingly negligible at later epochs. The $\mathrm{fb}-\mathrm{ff}$ components for 89-281 days are plotted in Figure 18. At 500 days the, fb-ff flux is too weak to appear on the 500 days plot which has been scaled to allow easy comparison with the earlier epochs. A more comprehensive estimation of the fb-ff flux, taking into account the temperature and ionization gradients, is beyond the scope of this paper.

As noted in Section 3.4.3.1, in the wavelength region $(\lambda>$ $15 \mu \mathrm{m})$ where the cold component makes a significant contribution $(>20 \%)$ to the total flux, the IS IR echo maintained a near-constant luminosity $(15-150 \mu \mathrm{m})$ of $\sim 2.0 \times 10^{38} \mathrm{erg} \mathrm{s}^{-1}$ between 89 days and 1393 days. Moreover, in setting the IS IR model to reproduce the long-wave excess at the latest epochs, when the fb-ff flux was negligible, it was found that a satisfactory match to the long-wave $(\lambda>15 \mu \mathrm{m})$ excess was automatically achieved for all epochs. Consequently, the fb-ff flux could make, at most, only a minor contribution even at the earliest epochs. We conclude that any ff emission was too weak to account for the cold component at any epoch.

\subsubsection{The Warm Component}

3.4.4.1. Late-time Optical and NIR Line Profiles. In Section 2.4, we described the evolution of optical and NIR line profiles in a number of species. In particular, we noted the development of a sharp suppression in the red wing, suggesting dust formation causing obscuration of the far side of the ejecta. Could this dust also be responsible for the warm component at later epochs? In this and the next sections, we explore this possibility. Here we examine the distributions of dust that might give rise to the late-time optical/NIR line profiles. In Section 3.4.4.2, we shall then test the hypothesis that the same dust distributions are responsible for the warm component. We modeled the line profiles of $\mathrm{H} \alpha, \mathrm{Pa} \beta,\left[\mathrm{OII}_{\mathrm{I}} 6300 \AA\right.$, [Fe II] $7155 \AA$, and [Fe II] $12567 \AA$. The period 461-925 days was studied since this was mostly overlapped by the 500-1393 days during which the blackbody analysis and more detailed studies of the MIR emission (Section 3.4.4.2) suggest that substantial quantities of dust were present in the ejecta. We note (Table 6) that the $\mathrm{H} \alpha$ line widths toward the end of the bright plateau phase greatly exceeded those observed on 461 days and later, implying that there was a negligible contribution of any light echo of the early-phase profiles to the late-time profiles.

We did also examine the blue asymmetry of the $\mathrm{H} \alpha$ and [Fe II] $7155 \AA$ profiles at a much earlier epoch ( 283 days) but were unable to achieve a satisfactory match using the model adopted for later epochs. In any case, as already demonstrated in Section 3.4.2, we were able to account for the 251/81 days IR excess as thermal emission from dust formed in the CDS. Moreover, Chugai et al. (2005) and Chugai (2006) successfully explained the $\mathrm{H} \alpha$ profile up to $\sim 300$ days by invoking the emergence of an intrinsic asymmetric, bipolar core (i.e., no dust involved). We conclude that there is no evidence for the formation of new ejecta dust earlier than 461 days.

The line-profile red wings exhibit increasingly abrupt declines after $\sim$ one year (Figure 10) suggesting the condensation of attenuating dust. In contrast, the extended blue wings exhibit little sign of developing suppression. This behavior points to the attenuating dust lying at low line-of-sight velocities, such as a face-on disk-like distribution centered on the SN center of mass. Nevertheless, in our initial considerations of possible dust configurations we included the case of a spherically symmetric dust sphere. Our line-profile model comprises a homologously expanding sphere of gas responsible for most of the observed line flux, with the emission being attenuated by a dust zone lying concentrically with the gas sphere. The dust zone is also assumed to participate in the expansion. The gas emissivity is assumed to have a power-law dependence with radius. 

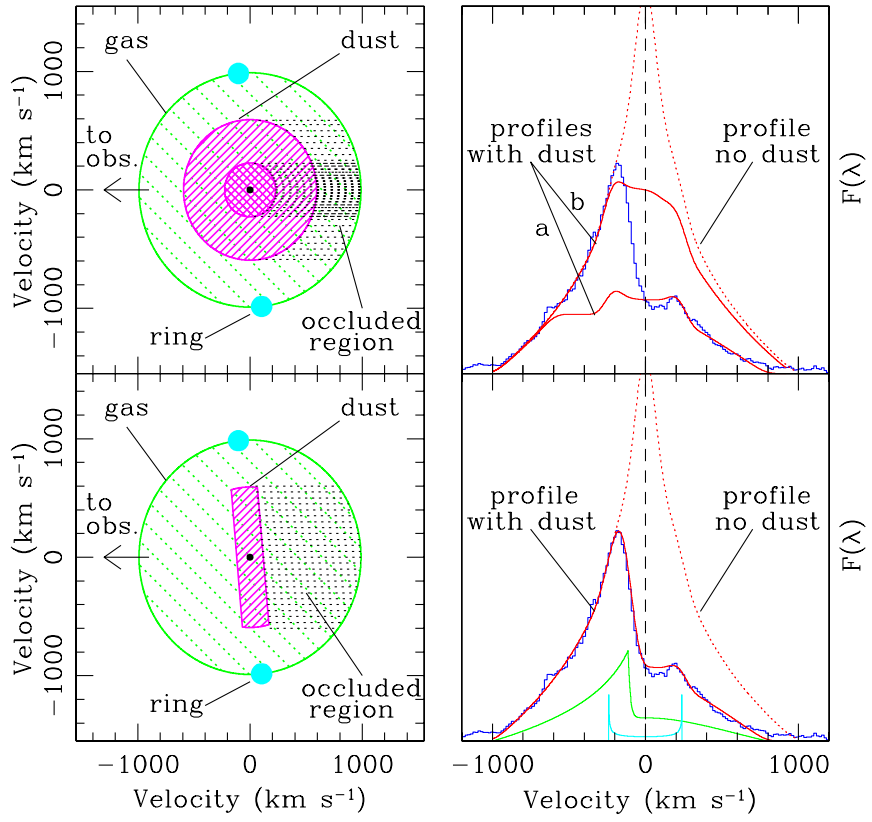

Figure 16. Illustration of the line-profile models. The model profiles are derived from a homologously expanding, emitting gas sphere attenuated by an embedded, opaque dust distribution. A minor additional contribution to the model is provided by a thin, expanding concentric ring of emitting gas oriented with the normal to the ring plane at $6^{\circ}$ to the line of sight. The gas-dust configurations are illustrated in the LH panels which show sections through the SN system in the plane defined by the thin ring axis and the line of sight. The gas sphere is shaded green and the attenuating dust shaded magenta. The thin ring, viewed edge-on in the figure, is represented by two large cyan dots. The region of line emission occluded by the dust is shown by the black shading. The expansion velocities are indicated by the axes. The final model profiles are obtained by the combination of the attenuated sphere and unattenuated ring spectra. The resulting spectrum is then smoothed to the spectral resolution. The RH panels show the resulting model line profiles (solid red), plotted in velocity space, compared with the observed spectrum (blue). Also shown (dotted red) is the line profile that would result in the absence of dust attenuation. In this illustration, we compare the model profiles with the observed 895 days [O I] $6300 \AA$ line. In this case, the gas sphere emissivity declines as $r^{-1.9}$. The upper panels illustrate the case where the dust is distributed as an opaque, concentric sphere. Two sizes of sphere are considered. Profiles $a$ and $b$ (upper RH panel) correspond, respectively, to the large and small dust spheres (upper LH panel). It can be seen that neither reproduce the observed line profile. The lower panels illustrate the case where the dust is composed of amorphous carbon and is distributed as a uniform density, nearly face-on concentric disk with $\tau=18$ perpendicularly through the disk plane. This optical depth is the value required for the model to simultaneously match the line profile and the contemporaneous MIR continuum (see Figure 19). While the disk is shown as being coplanar with the ring, for ease of computation the disk is taken to be exactly face-on. Shifting the disk tilt to match that of the ring would have had only a small effect on the model profile. No correction is made for minor edge effects at the disk's outer limit (radius). Also shown (lower RH panel) are the intrinsic line profiles from the attenuated gas sphere (green solid line) and the thin ring (cyan solid line). The thin ring emission is not affected by the dust. For clarity, both of these intrinsic profile plots have been scaled downward in flux by the same amount relative to the total profile. It can be seen that the final dust-disk model profile provides an excellent match to the data.

(A color version of this figure is available in the online journal.)

Several arrangements of dust were examined. These were (1) a uniform opaque sphere (Figure 16, upper panels), (2) a face-on opaque ring, and (3) a thin face-on disk (Figure 16, lower panels) whose opacity was uniform or varied radially as an $r^{-\delta}$ power law along the disk plane but was always uniform normal to the disk plane. (For the disks, "opacity" refers to the value normal to the disk plane.) For the radially varying version of (3), a power-law index of $\delta=1.9$ was chosen as typical of the power-law indices determined for the gas emission profiles. Configurations (1)-(3) were initially tested against the
895 days optical profiles since these were (1) of the highest available resolution and (2) corresponded to a time by which the SN spectral continuum was overwhelmingly dominated by thermal emission from dust. Configurations (1)-(2) could not reproduce the observed line profiles. This is illustrated in Figure 16 (upper panels) where we show two examples of the line profile produced by an opaque, concentric dust sphere, compared with the observed [O I] $6300 \AA$ profile at 895 days. Only configuration (3) - the dust disk - was able to reproduce the observed profiles. Within this configuration, we examined cases where the dust distribution (1) extended beyond the gas limit, (2) ceased abruptly at a given velocity within the gas sphere, or (3) extended uniformly to a given velocity, $v_{\text {duni }}$, and then declined as a power law to the edge of the gas sphere. Case (1) failed to reproduce the observed line profiles. Cases (2) and (3) are discussed below.

As described in Section 2.4, by 895 days and 925 days, a "secondary," weaker, redshifted peak had also appeared in the $\mathrm{H} \alpha$ and $\left[\mathrm{O}_{\mathrm{I}}\right] 6300 \AA$ profiles, suggesting that a fraction of the line flux originated in an emission zone centered on the SN and having the geometry of an expanding ring, jet, or cone. The second peak had a redshift of only +160 to $+170 \mathrm{~km} \mathrm{~s}^{-1}$. Given the preference for a thin face-on disk of attenuating dust to account for the asymmetry of the line profiles, a natural explanation for the low velocity of the second peak is that its source was actually at a velocity comparable to that of the gas sphere, but moving in a way which was roughly coplanar with the dust disk. Consequently, the low inclination angle led to the low observed redshift in the second peak. The simplest geometry which accounts for the second peak is a ring-like emission zone whose plane lays at a small angle to the "faceon" plane. A cone or jet geometry is more problematic. If we insisted on maintaining axial symmetry then a cone/jet would have to be normal to the disk but with a mysteriously low velocity. Alternatively, a cone/jet lying near the disk plane, while possibly allowing a high intrinsic velocity, would have the unattractive feature of breaking the axial symmetry. Therefore, to account for the second peak, we added a thin, near-coplanar ring of emitting gas to the line-profile model (cf. Gerardy et al. 2000; Fransson et al. 2005, for the case of SN 1998S). The ring was assumed to participate in the overall homologous expansion of the gas.

We set the intrinsic ring velocity equal to the fastest moving gas observed viz. hydrogen at $2400 \mathrm{~km} \mathrm{~s}^{-1}$ as indicated by the $\mathrm{H} \alpha$ blue wing on 895 and 925 days, although higher velocities could have been used. Consequently, the ring emission is unattenuated by the dust. For ease of computation, we retained a face-on disk $\left(i=0^{\circ}\right)$. Tilting the disk to become exactly coplanar with the ring would have had only a small effect on the line profile.

In matching the model to the observed profiles, the free parameters for the gas are the maximum (outer) velocity of the gas sphere, $v_{\text {gmax }}$, the gas emissivity scaling factor and powerlaw index $\beta$, the inclination, $i_{r}$, of the ring component assuming an intrinsic expansion velocity of $2400 \mathrm{~km} \mathrm{~s}^{-1}$, and the ring component emissivity assumed uniform. In addition, the overall wavelength positions of the observed profiles were allowed to vary by small amounts (see below). For ease of comparison, the dust-disk dimensions were also expressed as velocities within the homologous expansion. Thus, for case (2) dust disk the free parameters are the maximum radial velocity of the dust disk, $v_{\mathrm{dmax}}$, the maximum velocity, $v_{\mathrm{dth}}$, perpendicular to the disk plane and the magnitude of the dust radial density power-law 
index, $\delta$. Multiplication of $v_{\mathrm{dmax}}$ and $2 v_{\mathrm{dth}}$ by the epoch yields, respectively, the disk radius and thickness at that time. For case (3) $v_{\text {dmax }}$ is replaced with $v_{\text {duni }}$. We adopted amorphous carbon as the grain material (see Section 3.4.4.2) and a power-law grain-size distribution, index $m=3.5$ (Mathis et al. 1977), with $a_{(\min )}=0.005 \mu \mathrm{m}$ and $a_{(\max )}=0.05 \mu \mathrm{m}$. The optical depth normally through the disk was then calculated. For example, for case (2) the optical depth for emission from gas lying behind the disk at wavelength $\lambda$, epoch $t$, and radial velocity $v_{d}, \tau_{\lambda}\left(t, v_{d}\right)$, is given by

$$
\tau_{\lambda}\left(t, v_{d}\right)=\frac{4}{3} \pi \rho \kappa_{\lambda} k \frac{1}{4-m}\left[a_{(\max )}^{4-m}-a_{(\min )}^{4-m}\right] v_{d}^{-\delta} 2 v_{\mathrm{dth}} t^{-2},
$$

where $k$ is proportional to the grain number density, at a fiducial epoch, to the radial velocity and to the grain radius, $\rho$ is the grain material density, and $\kappa_{\lambda}$ is the mass absorption coefficient of the grain material at wavelength $\lambda$. For emission from gas lying within the disk, the same formula is used but replacing $2 v_{\text {dth }}$ with the velocity equivalent to the path from the emission point to the near side of the disk. The disk thickness was always $<20 \%$ of the disk diameter and so edge effects at the disk's outer limit were assumed to be negligible.

The gas emission profiles (i.e., those for the attenuated sphere and unattenuated ring) were individually convolved with the appropriate slit PSF. The PSF FWHM values were 6.5, 4.2, $6.5,1.25$, and $1.75 \AA$ for $461,467,554,895$, and 925 days, respectively. The two components were then summed to form the final model profile which was compared with the observed profile. The profile model (case (2) disk) was adjusted to match the observed profiles as follows. The parameters $v_{\text {gmax }}, \beta$, and the gas sphere emissivity scaling factor were adjusted to provide a match to the blue wing of the profile. Then, for a given value of $\delta(0$ or 1.9$), v_{\mathrm{dth}}$ (equivalent to the disk half-thickness) together with the grain number density scaler, $k$, were adjusted to reproduce the sharp-decline section of the profile. The velocity $v_{\text {dmax }}$ was varied to reproduce the suppressed red wing. The gas ring emissivity and inclination were then adjusted to reproduce the secondary peak. Some iteration of all the model parameters was necessary to reach the final profile match. For the profile model (case (3) disk), $v_{\text {dmax }}$ was replaced with $v_{\text {duni }}$, and $\delta$ was allowed to vary to arbitrarily large steepness.

The offset of the sharp-decline section of the profiles from zero velocity was only about $-100 \mathrm{~km} \mathrm{~s}^{-1}$ after correction for the $+221 \mathrm{~km} \mathrm{~s}^{-1}$ of the heliocentric velocity. Consequently, the match to the sharp-decline section of the profile (i.e., determination of the disk half-thickness) could be significantly affected by errors in individual wavelength measurements or intrinsic variations in the distributions of different gas species. Forcing a match in each case by letting the wavelength position of each observed profile to vary by small amounts, we were able to estimate the disk half-thickness and its uncertainty. For a heliocentric velocity of $+221 \mathrm{~km} \mathrm{~s}^{-1}$, we obtained a disk halfthickness equivalent to $v_{\mathrm{dth}}=+112 \pm 18 \mathrm{~km} \mathrm{~s}^{-1}$. There was no significant variation in $v_{\text {dth }}$ during the period covered by the profile study. Note that a change in the heliocentric velocity estimate would yield the same change in the disk half-thickness velocity.

As indicated above, we first carried out model matches to the 895 days profiles. It was found that the best case (2) matches were obtained when the disk was highly opaque (say, $\tau>5$ ) at all radial locations. For case (3), comparably good matches were achieved by setting the uniform zone at a high opacity $(\tau>5)$ and $\delta$ at values steeper than $\sim 10$-i.e., the dust density beyond the uniform zone had to be extremely steep. Less steep declines tended to suppress the visibility of the central minimum. We conclude that the dust disk was highly opaque in the optical region but terminated abruptly at an approximately fixed radius (see Section 3.4.4.2). Given that this condition was indicated by both cases (2) and (3), we abandoned the more complicated case (3) model and completed the analysis for all the 461-925 day spectra using only case (2)-i.e., a dust density which was uniform or declined radially as $r^{-1.9}$ and which terminated abruptly at $v_{\text {dmax }}$.

The models for 895 days were initially adjusted to determine the minimum opacity - that is, the minimum dust mass that was needed to provide a satisfactory match to the observed profile. The dust masses were obtained from the following:

$$
M_{d}=\frac{8}{3} \pi^{2} \rho \kappa_{\lambda} k \frac{1}{4-m}\left[a_{(\max )}^{4-m}-a_{(\min )}^{4-m}\right] \frac{1}{2-\delta} v_{\mathrm{dmax}}^{2-\delta} 2 v_{\mathrm{dth}} .
$$

At 895 days, with a uniform disk $(\delta=0)$, the dust extended to $v_{\text {dmax }}=590 \pm 25 \mathrm{~km} \mathrm{~s}^{-1}$, i.e., $r_{\text {dmax }}=(4.6 \pm 0.2) \times 10^{15} \mathrm{~cm}$. The disk half-thickness was $19 \%$ of this. Simultaneous matches to all three profiles (H $\alpha$, [O I] $6300 \AA$, [Fe II] $7155 \AA$ ) required a minimum $\tau$ of 13 at $6300 \AA$, corresponding to a minimum dust mass of $0.27 \times 10^{-4} M_{\odot}$. Note that the high optical depth was demanded by the very sharp decline, unresolved even at the $1.25 \AA$ resolution of 895 days. Other parameters are $r_{\text {gmax }}=7.65 \times 10^{15} \mathrm{~cm}, \beta=1.9$, (ring flux $) /($ total flux $)=$ 0.07 , and ring tilt $=6^{\circ}$.

In using the dust-disk model to reproduce the contemporary MIR continua (Section 3.4.4.2), it was found that the opacities and dust masses for all lines and epochs had to be somewhat higher than the minimum values required to match the observed optical and NIR line profiles. For example, for [O I] $6300 \AA$ at 895 days and a uniform disk it was necessary to set $\tau \geqslant 18 \pm 3$, corresponding to a minimum dust mass of $(0.38 \pm 0.06) \times$ $10^{-4} M_{\odot}$. In the rest of this subsection, therefore, we present and consider results for profile matching incorporating dustdisk masses obtained by interpolating to the profile epochs the values obtained from the MIR continuum modeling. A sketch of case (2) [O I] $6300 \AA$ uniform $(\delta=0)$ disk model at 895 days is shown in Figure 16 (lower LH panel) together with an illustration (lower RH panel) of the model match (solid red line) to the observed profile (blue). Also shown are the intrinsic line-profile contributions from the attenuated gas sphere (green) and unattenuated gas ring (cyan) components, as well as the final profile (dotted red line) which would result if the attenuating dust were removed. In Figure 17, we show all the individual matches to the line profiles for 461-925 days. In general, good matches were achieved for epochs 554 days, 895 days, and 925 days. Poorer matches were obtained at 461 days and 467 days.

On 895 days and 925 days, the model did not reproduce the extended red wing seen in the [Fe II] $7155 \AA$ profile. This suggests an additional, faster moving component of iron and may be indicative of ${ }^{56} \mathrm{Ni}$ asymmetry or "bullets" in the initial explosion (e.g., Burrows et al. 1995). At the earliest two epochs (461 days and 467 days), the model matches are also poorer. Specifically, if the model was adjusted to match the full suppression of the extreme red wing, then it also overattenuated the less redshifted portion of the red wing; in other words, the steep red decline in the observed profile is generally less pronounced at these earliest epochs. Alternatively, matching to the less redshifted portion of the red wing meant that the full suppression of the extreme red wing was not reproduced. These points suggest that, during the 461-467 days period, dust 


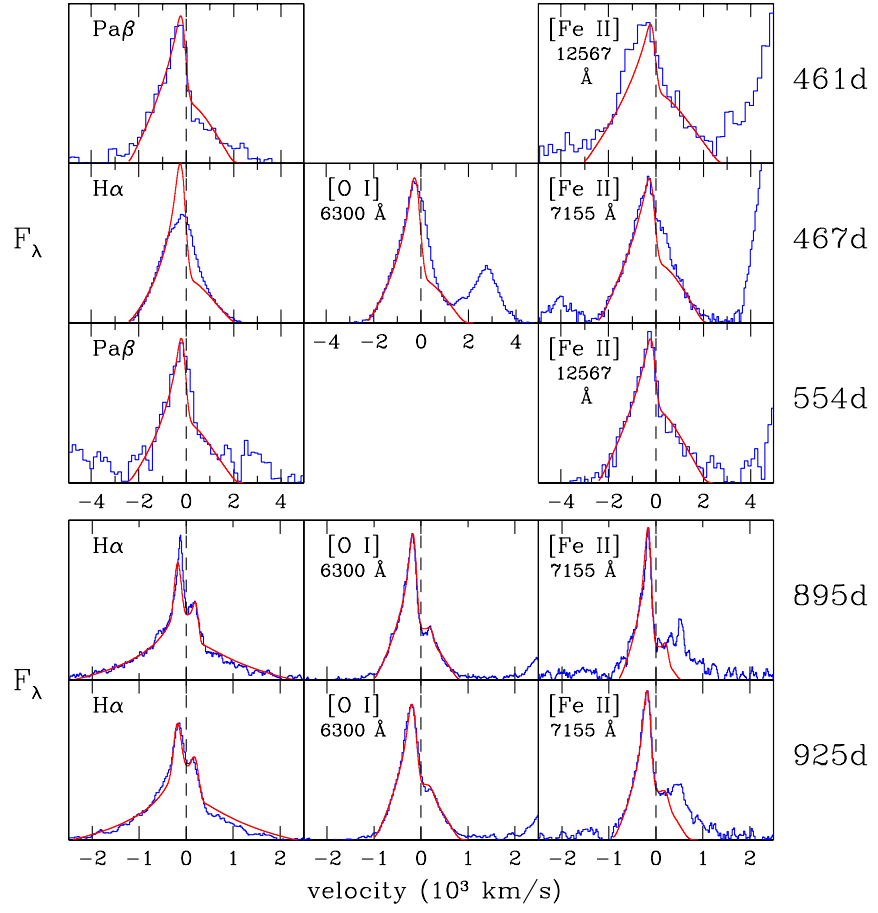

Figure 17. Individual dust-disk model matches (red) to observed line profiles (blue) of SN 2004dj for the case where $\delta=0$ (uniform dust density). The velocities of the observed spectra have been shifted by $-221 \mathrm{~km} \mathrm{~s}^{-1}$ to match the center-of-mass rest frame of the SN. The latest two epochs also include a small contribution from a ring of emission. Also, the velocity scale of these two epochs has been expanded to provide more detail. The matches incorporate dustdisk masses obtained by interpolating to the profile epochs the values obtained from the MIR continuum modeling. Between 554 days and 925 days, the dust mass increased from $0.25 \times 10^{-4} M_{\odot}$ to $0.4 \times 10^{-4} M_{\odot}$. The radius of the dust disk decreased slightly from $5.3 \times 10^{15} \mathrm{~cm}$ to $4.6 \times 10^{15} \mathrm{~cm}$. The disk halfthickness expanded from $0.45 \times 10^{15} \mathrm{~cm}$ to $0.90 \times 10^{15} \mathrm{~cm}$. For each profile, the optical depth had a value in the range $3<\tau<19$ (i.e., the disk was always of high optical depth).

(A color version of this figure is available in the online journal.)

formation was less complete and was not yet fully opaque over the whole disk. We therefore adjusted the models to reproduce the suppression of the extreme red wing, but recognize that the derived dust mass lower limits for 461-467 days may well be overestimated.

Between 554 days and 925 days, the minimum dust mass increased from $0.25 \times 10^{-4} M_{\odot}$ to $0.4 \times 10^{-4} M_{\odot}$. During this time, the radius of the dust disk appeared to actually decrease slightly from $5.3 \times 10^{15} \mathrm{~cm}$ to $4.6 \times 10^{15} \mathrm{~cm}$. We explored the possibility that this was due to a declining optical depth as the disk expanded-i.e., while the dust disk continued to expand, the optically thick/thin boundary declined in radius. We found that the requirement of a steep, sharp cutoff at the disk limit (see above) ruled out this explanation implying that the decrease in the dust radius was real. This issue will be considered further in Section 3.4.4.2.

The above analysis was repeated with $\delta=1.9$. Similar disk radii were obtained, but the minimum dust masses were $\sim \times 20$ larger than for $\delta=0$. This is as one would expect. To maintain the line-profile matches with $\delta=1.9$ required the maintenance of a high optical depth at the disk edge in the optical/NIR wavelength range. If we demand that the optical depths be the same at $r_{\mathrm{dmax}}$ for both $\delta=0$ and $\delta>0$ then it may be shown that $M_{d}(\delta) / M_{d}(0)=2 /(2-\delta)$, where $M_{d}(\delta)$ is the dust mass for a given value of $\delta$ and $M_{d}(0)$ is the mass for $\delta=0$. Thus, for $\delta=1.9, M_{d}(\delta) / M_{d}(0)=20$. We note that this assumes that the power law continues to the center, which is unlikely to be the case. Indeed for $\delta=2$ or more, the mass would be infinite. A more plausible behavior would be that the density law flattens toward the center. For example, if we assume that within $0.05 r_{\mathrm{dmax}}$ the dust density became uniform, with $\delta=1.9$ outside $0.05 r_{\mathrm{dmax}}$, then the total minimum dust mass would only be about a factor of three larger than for the totally uniform disk case-that is, about $10^{-4} M_{\odot}$

The model gas sphere expansion velocity remained at around $2400 \mathrm{~km} \mathrm{~s}^{-1}$ in hydrogen at all epochs. Similar velocities were obtained in [Fe II] $12567 \AA$ at 461 days and 554 days, and in [O I] $6300 \AA$ and [Fe II] $7155 \AA$ at 467 days. By 895 days and 925 days, the expansion velocities in [O I] $6300 \AA$ and [Fe II] $7155 \AA$ had fallen to less than $1000 \mathrm{~km} \mathrm{~s}^{-1}$, perhaps suggesting some stratification by element. At 554 days and earlier epochs, the ring flux was negligible. At the later epochs, the ring inclination is $6^{\circ}$ (i.e., close to face-on). The fractional contribution of the ring to the total observed flux never exceeded $20 \%$. Indeed, were it not for the dust disk, it is unlikely that the relatively weak ring emission would have been detected since it would have been swamped by the emission from the gas sphere (see Figure 16). We therefore regard the ring component as an interesting but minor effect.

We conclude from the line-profile analysis that, in the 461-925 days period, the mass of the dust disk and its radial extent can be small enough to be consistent with an origin as newly condensed ejecta dust. In Section 3.4.4.2, we shall show that the same dust disk can account for the MIR continua.

3.4.4.2. Later Phase IR Excess and Ejecta Dust Condensation. We have argued that, up to at least 281 days, the warm component could be fully accounted for in terms of an IR echo from a CDS. By 500 days, the warm component luminosity exceeded the CDS contribution by a factor of $\sim 20$. This "late IR excess," together with the steady shift of the peak emission of the SED to longer wavelengths in the period $\sim 250-1200$ days (Figures 3 and 5), suggests the appearance of an additional population of warm, but cooling dust. What is the location, distribution, and heating mechanism of this dust? Such IR emission can arise from (1) heating, by a number of possible mechanisms (see below), of new dust formed either in the ejecta, or in a late surge of dust growth within the CDS or (2) heating of pre-existing circumstellar dust by the early-time SN luminosity (a conventional IR echo), or possibly the forward shock traveling into undisturbed CSM beyond the CDS. We can dismiss immediately hypothesis (2).

1. An IR echo does not naturally produce the long delay (at least $\sim 300$ days) before the commencement of the warm component excess flux rise. To account for this within an IR echo scenario, we would have to invoke an ad hoc asymmetry in the CSM.

2. IR-echo or forward-shock heating would not account for the observed late-time red-wing suppression in the spectral line profiles discussed in Section 3.4.4.1. In contrast, redwing attenuation can easily be produced by dust formation in the ejecta. New CDS dust could conceivably also have produced such an effect provided that the dust did not lie completely outside the optical/IR emission zone.

3. Pre-existing CSM dust, whether heated by the SN luminosity or a forward shock, would not produce steepening of the optical-NIR decline rate simultaneously with the appearance of the late IR excess. In contrast, new dust in the ejecta or CDS can be indicated by optical-NIR 
steepening. In Figure 4 , after $\sim 470$ days the VRIJ light curves show weak evidence of steepening, providing a minor additional argument against a CSM IR echo origin for the later warm component, although the errors on the latest points are large. We also note that an alternative explanation for such steepening could be a faster-than-expected decrease in the $\gamma$-ray absorption relative to that invoked by Li et al. (1993) for the radioactive energy deposition in SN 1987A (Figure 4).

The above points, especially (1) and (2), leave us with hypothesis (1) viz. that the late IR excess is due to emission from new dust formed in either the ejecta or the CDS. The laterera appearance of the red-wing suppression coincides roughly with the emergence of the late IR excess, suggesting that the same dust could have been responsible for both the optical/NIR attenuation effects and the rise of the MIR emission. That this dust formed in the ejecta rather than the CDS is indicated by the fact that the extent of the attenuating dust derived from latetime line-profile analysis was comparable to that of the MIRemitting dust as derived from blackbody analysis; for example, at 859 days the blackbody radius is $3.3 \times 10^{15} \mathrm{~cm}$ compared with a profile-derived radius of $(4.4 \pm 0.2) \times 10^{15} \mathrm{~cm}$ at 895 days. Moreover, the line-profile analysis demonstrates that the dust was of high opacity in the optical/NIR and distributed as a near face-on disk lying concentrically with the SN center of mass. Attenuation by a CDS could not have produced the late-time line-profile behavior.

In view of the above points, we shall now give detailed consideration to the scenario where the warm component emission originated in newly formed ejecta dust. Such dust could be heated by a number of mechanisms including radioactive decay, ionization freeze-out effect, embedded pulsar, or reverseshock radiation. In the case of SN 2004dj, we can rule out radioactivity as the principal heating mechanism of any putative new ejecta dust. Inspection of Table 7 shows that, as early as 500 days, the warm component flux exceeded the total deposited radioactive luminosity by a factor of two growing to a factor of $\sim 450$ by 1393 days. Indeed, by the latest epoch, the warm component luminosity was still as high as $\sim 4 \times 10^{38} \mathrm{erg} \mathrm{s}^{-1}$. We also rule out the freeze-out effect as the main energy source (Clayton et al. 1992; Fransson \& Kozma 1993) since, at $\sim 1400$ days, its contribution would only be $\sim 5 \times 10^{36} \mathrm{erg}$.

Power input from an embedded pulsar, via a pulsar wind nebula, is another possibility (Woosley et al. 1989; Chevalier \& Fransson 1992). Chevalier \& Fransson predicted that a distinctive feature of such a source would be the presence of certain high-ionization lines. The earliest epoch they studied was at 1500 days. The nearest of our optical/NIR spectra to this epoch are those at 895 days and 925 days. Of the high-ionization lines for which Chevalier \& Fransson make luminosity predictions, only [O III] 4959/5007 ^ was covered by these spectra. We co-added the spectra at the two epochs and searched for the stronger [O III] $5007 \AA$ component. A broad, low $\mathrm{S} / \mathrm{N}$ emission feature was detected with a dereddened luminosity of $(0.8 \pm 0.2) \times 10^{36} \mathrm{erg} \mathrm{s}^{-1}$. This is about half the luminosity predicted by Chevalier \& Fransson. A serious difficulty is that the redshift-corrected position of the feature lies about $+400 \mathrm{~km} \mathrm{~s}^{-1}$ from where it would be if due to an [O III] $5007 \AA$ line subject to the same dust attenuation deduced in other lines (Section 3.4.4.1). Even without dust attenuation, the feature would still be $+200 \mathrm{~km} \mathrm{~s}^{-1}$ too far to the red. This, together with the low $\mathrm{S} / \mathrm{N}$, leads us to conclude that there is no persuasive evidence for the presence of pulsar-driven high- ionization features in the latest spectra of SN 2004dj. This leaves us with reverse-shock heating of the ejecta following the ejectaCSM collision as the most promising mechanism for the latetime energy source.

To test further the hypothesis that the same dust was responsible for the late-time line-profile red-wing suppression and the MIR emission, we modeled the IR emission over a range of late-time epochs using the same dust-disk configuration as was employed in the line-profile analysis. The emission was derived following a similar procedure to that used in the isothermal dust model by Meikle et al. (2007) and Kotak et al. (2009). The resulting flux was then added to the other continuum components and the net model compared with the observed continua.

We considered the thermal radiation from a warm, isothermal, face-on disk of dust located symmetrically about the SN center of mass. We shall refer to this as the isothermal dust-disk model (IDDM). The disk radius is $r_{\mathrm{dmax}}$ and, as in Section 3.4.4.1, the dust number density declines as $r_{d}^{-\delta}$, where $\delta$ is set as 0 or 1.9. As mentioned above, the disk thickness was $<20 \%$ of the disk diameter and so we judged that a thin-disk treatment would provide an adequate means of estimating the flux from the disk (i.e., edge effects are ignored). The observed IDDM flux, $d F_{\lambda}\left(t, r_{d}\right)$, at wavelength $\lambda$ and time $t$ from an elemental ring lying between radii $r_{d}$ and $r_{d}+d r_{d}$ is

$$
d F_{\lambda}\left(t, r_{d}\right)=2 \pi r_{d} d r_{d} D^{-2} B_{\lambda}(t)\left(1-\exp \left(-\tau_{\lambda}\left(t, r_{d}\right)\right)\right),
$$

where $D$ is the distance of the $\mathrm{SN}, B_{\lambda}(t)$ is the Planck function, and $\tau_{\lambda}\left(t, r_{d}\right)$ is the optical depth perpendicularly through the disk. The total flux is then found by integrating from $r_{d}=0$ to $r_{d}=r_{\mathrm{dmax}}$. The dust mass is obtained from Equation (4). Amorphous carbon grains were assumed (silicate grains are discussed below). As explained before, during the 89-281 days period ejecta dust formation was unlikely and in any case the early IR excess was explainable as emission from CDS dust. Therefore, the IDDM was introduced at 500 days and used at all subsequent epochs. By 652 days the CDS IR emission was negligible and so was not included in the modeling of this or subsequent phases. We first describe the results with $\delta=0$ in the IDDM. The matches together with the observations are shown in Figures 18 and 19. The overall model parameters are listed in Table 8.

The three MIR observation epochs 500 days, 652 days, and 859 days lay within the timespan of the line-profile analysis. We therefore imposed the constraint that the disk radius had to be consistent with those values derived from the line profiles - that is, $r_{\mathrm{dmax}} \approx 5 \times 10^{15} \mathrm{~cm}$ and $r_{\mathrm{dth}}=v_{\mathrm{dth}} \times t$, where $v_{\mathrm{dth}}=$ $+112 \mathrm{~km} \mathrm{~s}^{-1}$, with $k$ (and therefore $M_{d}$ ) being constrained by the demand that the disk have a high optical depth in the optical/ NIR region (Section 3.4.4.1). In practice, matching the IDDM to the MIR continuum demanded optical depths which were higher than the minimum values obtained from the line-profile analysis. The disk parameters for the specific MIR epochs were set by linear interpolation of the line-profile-derived values for $r_{\mathrm{dmax}}$ and $r_{\mathrm{dth}}$. The $k$ parameter could take values at or above those set in the line-profile analysis. Only $T_{d}$ was a completely free IDDM parameter. By 500 days the MIR continuum up to $\sim 20 \mu \mathrm{m}$ was overwhelmingly due to the warm dust disk, with the CDS component yielding no more than $\sim 8 \%$ of the total flux at any wavelength. For $\lambda \gtrsim 20 \mu \mathrm{m}$, the IS IR echo dominated. Also from 500 days onward the hot continuum and fb-ff contributions to the MIR were negligible. In other words, from 500 days onward, only the dusty disk and IS IR echo made significant contributions to the total MIR continuum. Consequently, and in 


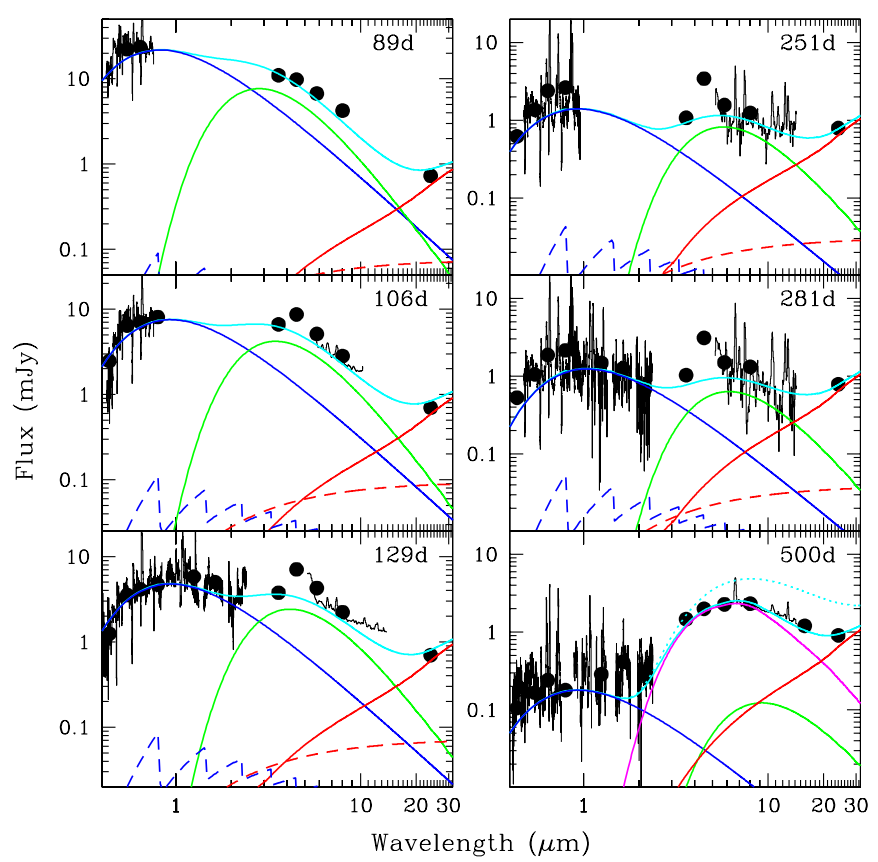

Figure 18. 89-281 days: model continua comprising hot blackbody (solid blue), free-bound (dashed blue), free-free (dashed red), CDS IR echo (green), IS IR echo (solid red), and total flux (solid cyan). The observed spectra and contemporary photometry (dots) of SN 2004dj are plotted in black. 500 days: as for earlier epochs but with the addition of IR emission from a warm disk of dust (magenta). The dotted cyan line represents the model continuum when the IDDM is replaced with a blackbody adjusted to match the short-wave region of the MIR continuum. At this epoch the $\mathrm{ff}$ and fb fluxes are too weak to appear on the plot.

(A color version of this figure is available in the online journal.)

order to show the MIR behavior in more detail, the optical/NIR region is not shown in the plots for 652-1393 days (Figure 19).

In spite of the line-profile constraints on $r_{\mathrm{dmax}}$ and $r_{\mathrm{dth}}$, for epochs 500 days, 652 days, and 859 days, we were nevertheless able to obtain fair matches to the observed continuum (Figures 18 and 19). It was found that the matches required the dust to be optically thick $(\tau>1)$ in the optical/NIR region, but optically thin in the MIR region. A blackbody spectrum matched to the shorter MIR wavelengths overproduced the flux at longer wavelengths. This is illustrated in Figure 18 (500 days) and Figure 19 as dotted cyan lines. This restriction, together with the fixed values for $r_{\mathrm{dmax}}$ and $r_{\mathrm{dth}}$, allowed us to obtain specific values, not limits, for $T_{d}, \tau$, and $M_{d}$. At 500 days the ejecta dust mass was $(0.22 \pm 0.02) \times 10^{-4} M_{\odot}$, with $T_{d}=650 \pm 15 \mathrm{~K}$. The optical depths through the disk were $\sim 10$ in the $V$ band and $0.45 \pm 0.05$ at $10 \mu \mathrm{m}$. By 859 days the dust mass had increased to $(0.33 \pm 0.05) \times 10^{-4} M_{\odot}$, while $T_{d}$ fell to $570 \pm 15 \mathrm{~K}$. A particular value of this 500-859 days study is that it demonstrates that the same dust-disk parameters can account for the line profiles and the MIR continuum. This adds considerable weight to our contention that the two disks are one and the same. Moreover specific values, not limits, for the dust masses and temperatures were determined for the 500-859 days period.

As the SN aged between 500 days and 996 days, it was found that the IDDM steadily approached the blackbody case. Moreover, there were no line profiles available after 925 days. Linear extrapolation to 996 days of the line-profile-derived values for $r_{\mathrm{dmax}}$ gave $r_{\mathrm{dmax}} \sim(4 \pm 1) \times 10^{15} \mathrm{~cm}$. The model match yielded dust with a high optical depth in the optical/ NIR but with $\tau \sim 1$ in the MIR. The dust mass was $M_{d} \approx$ $0.5 \times 10^{-4} M_{\odot}$ and $T_{d}=520 \pm 30 \mathrm{~K}$.

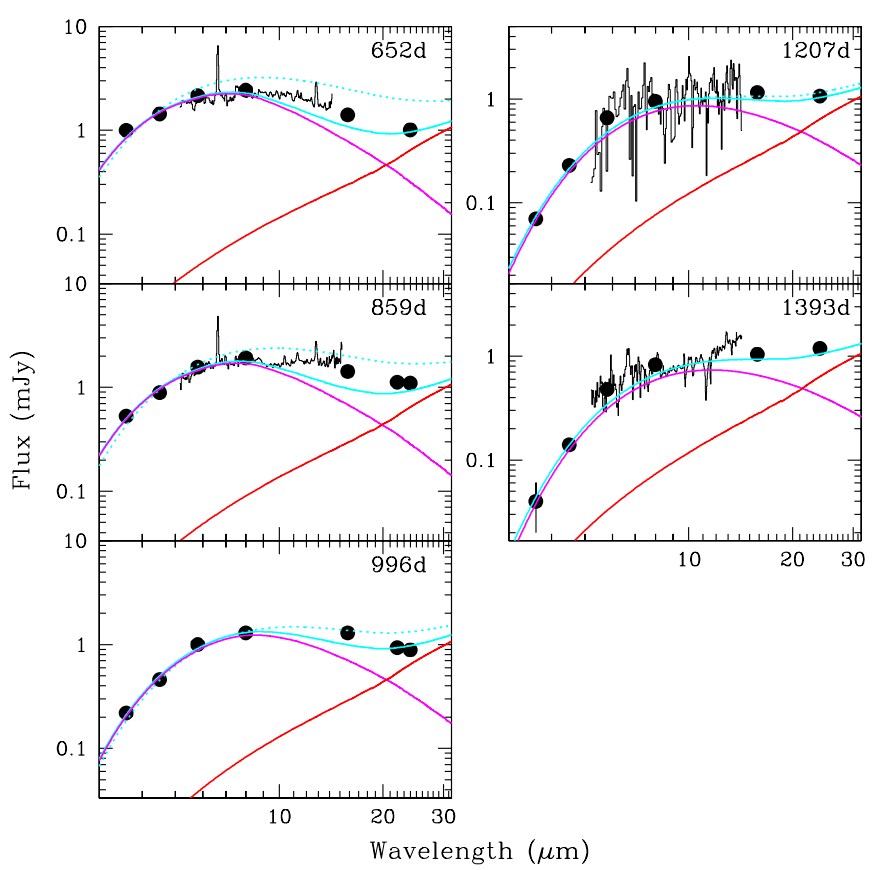

Figure 19. Model continua at epochs 652-1393 days, comprising IR flux from a warm disk of dust (magenta), IS IR echo (red), and total flux (solid cyan). The dotted cyan line represents the model continuum when the IDDM is replaced with a blackbody adjusted to match the short-wave region of the MIR continuum. By 1393 days the blackbody has fully merged with the IDDM; i.e., the model is effectively opaque over the whole observed wavelength range. The observed spectra and contemporary photometry (dots) of SN 2004dj are plotted in black. A hot blackbody plus free-bound and free-free continua were also included in the matches but these had a negligible effect on the MIR continua. Consequently, and in order to show the MIR behavior in more detail, the optical/NIR region is not shown. Estimates of the hot and fb-ff component contributions are given in Tables 8 and 9.

(A color version of this figure is available in the online journal.)

By epochs 1207 days and 1393 days, we found that the MIR continuum was best reproduced by allowing a continuing increase in the IDDM optical depth such that the disk was optically thick at all observed wavelengths (see Table 8). Indeed, given the uncertainties in the observed fluxes, we cannot rule out a totally opaque disk at all wavelengths covered. Consequently, the IDDM could provide only dust mass lower limits of $1.0 \times 10^{-4} M_{\odot}$ at 1207 days and $1.5 \times 10^{-4} M_{\odot}$ at 1393 days. The 1207 and 1393 day plots shown in Figure 19 are with $r_{\text {dmax }}$ and $T_{d}$ set at the limiting values (Table 8 ).

In Table 9, we show the luminosities of the IDDM $(\delta=0)$ components compared with the radioactive deposition power of $0.0095 M_{\odot}$ of ${ }^{56} \mathrm{Ni}$, including ${ }^{56} \mathrm{Ni}$ decay. The IS IR echo component is excluded since it is powered primarily by the $\mathrm{SN}$ peak luminosity. The luminosity of the thin disk, $L_{\mathrm{IDDM}}$, is approximated by

$$
L_{\mathrm{IDDM}} \approx 2 \pi R_{d}^{2} \pi B_{v}\left(1-\exp \left(-2 \tau_{\nu}\right)\right) .
$$

As already indicated by the BLC analysis (Section 3.2), the post-30 days evolution of SN 2004dj falls into three phases. At 89 days (about half-way down the plateau edge), the luminosity is still dominated by the shock-ionized ejecta, with radioactive decay contributing a small proportion of the total. There is then a short period just after the end of the plateau when radioactive decay deposition dominated the luminosity. This is supported by the fact that at 129 days, the radioactive luminosity, $L_{\mathrm{rad}}$, is highly similar to the total continuum model luminosity, $L_{\text {total }}$. At the nebular phases of 251 days and 281 days, the small excess 
in $L_{\text {rad }}$, relative to $L_{\text {total }}$, presumably went into powering the line emission which is not included in the model. By 500 days, in spite of the exclusion of much of the line luminosity from the model, $L_{\text {total }}$ is $\times 2.5 L_{\text {rad }}$, rising to $\times 200 L_{\text {rad }}$ by 1393 days. As argued above, the most plausible additional power source available at this stage is a reverse shock.

Model matches were also carried out with $\delta=1.9$ in the IDDM component. Similar dust parameters were found to those obtained for $\delta=0$. The only significant difference was that the dust masses were $\times 3$ larger, assuming a uniform density distribution within the inner $0.05 r_{\mathrm{dmax}}$ (cf. Section 3.4.4.1). These larger values are due to the growing proportion of dust mass concentrated in the optically thick region of the disk.

We also considered a dusty disk of warm silicate grains, with the radii and thickness determined by the line-profile analysis as before. The demand that the disk should be of optical depth in the optical/NIR up to its edge meant that the replacement of amorphous carbon dust with silicate dust had no effect on these dimensions. The problem with silicate dust continuum matching is the absence of the $8-14 \mu \mathrm{m}$ silicate feature in the observed continua during the period when the dust was optically thin in the MIR, up to 996 days. Attempts to suppress the feature in the IDDM by increasing the dust mass and hence the optical depth yielded a continuum that was too bright. Only for 1207 days and 1393 days was silicate dust able to reproduce the observed continuum. This is not surprising since, by these two epochs, the warm ejecta dust was close to being opaque over the MIR range observed. Between 500 days and 996 days, the proportion of silicate grains by mass could have been no more than $20 \%$ and was usually significantly less than this. Indeed, the data were always consistent with there being no silicate grains at all. We also note the absence of the $\mathrm{SiO}$ feature at 7.5-9.3 $\mu \mathrm{m}$. $\mathrm{SiO}$ formation is a necessary step on the way to silicate grains (Todini \& Ferrara 2001; Nozawa et al. 2003).

The points made in the previous paragraph argue against a substantial amount of silicate dust in the ejecta of SN 2004dj. The absence of silicate grains is consistent with the presence of strong $\mathrm{CO}$ fundamental and first overtone emission in the period $\sim 100$ days to $300-500$ days. A high $\mathrm{C} / \mathrm{O}$ ratio in the ejecta could result in most of the oxygen being absorbed as $\mathrm{CO}$ leaving behind an excess of carbon to provide carbon grains, but little oxygen to provide silicate grains. However, in the SN environment, the net grain population can be affected by factors in addition to the $\mathrm{C} / \mathrm{O}$ ratio. These include molecule destruction by high-energy electrons, charge transfer reactions, and ejecta density (Liu \& Dalgarno 1996; Nozawa et al. 2003; Deneault et al. 2006). Nevertheless, we conclude that the dust grains in SN 2004dj were predominantly composed of non-silicate material.

The success in reproducing the MIR continua using the same dust disk as was invoked to explain contemporary line profiles tends to support the curious result from Section 3.4.4.1 that, rather than expanding, the radius of the dust disk actually shrunk by a small amount. Indeed, if we include the disk radii for 1207 days and 1393 days derived from the IDDM matches (Table 8, Column 7) we find a shrinkage of $27 \%$ since 500 days. In SN 2004et (Kotak et al. 2009), it was found that the dust radius remained roughly constant. The explanation offered was that the dust was contained within an optically thin cloud of optically thick, pressure-confined clumps. But the availability of late-time optical/NIR spectra for SN 2004dj and the profile modeling presented here shows that a clumping explanation for the non-expanding dust-disk radius would not work. We note that, during the 500-859 days period in SN 2004dj, the product of the blue-wing, half-maximum (BHM) velocities and epoch for the line profiles, $R_{\mathrm{BHM}}$, yields a roughly constant value of $\sim 4 \times 10^{15} \mathrm{~cm}$ (see Table 6) - similar to that obtained for the dust-disk radius. This coincidence may imply that the extent of both the dust and the bulk of the ejecta gas emission was physically constrained within a radius of $\sim 5 \times 10^{15} \mathrm{~cm}$.

We suggest that the apparent shrinkage of the dust-disk radius may have been due to the presence and inward motion of the reverse shock such that the shock position defined the disk radius. Dust formation could have continued within the disk with the dust taking part in the overall ejecta outflow, but as the ejecta passed through the reverse shock it would have been destroyed. There would have been a net increase in dust mass with time, as observed, if the dust growth rate within the disk exceeded the destruction rate. A possible difficulty with this scenario is that the dust mass appeared to continue to grow right up to the final epoch at 1393 days. This is rather later than dustformation studies suggest. Todini \& Ferrara (2001) find that all grain condensation would be complete by 800 days. Moreover, both Todini \& Ferrara (2001) and Nozawa et al. (2003) find that carbon dust condensation is complete in not much more than one year. An alternative explanation, therefore, might be that the dust mass in SN 2004dj did not increase after $~ 400$ days, but rather that the density gradient of the outer region of the disk was actually steeper than $r^{-2}$. Thus, as the ejecta expanded, the dust density at a fixed location in the outer region would have grown, yielding an apparently higher total dust mass-i.e., more dust emerged from the optically thick inner regions.

Radiation from the reverse shock could have been primarily responsible for heating the dust. As more and more ejecta passed through, the shock and its radiation would have weakened causing the dust to cool, as observed. The reverse shock may also have been responsible for the approximate constancy of the radius of the line emitting gas sphere. Further examination of the reverse-shocked dust-disk hypothesis is beyond the scope of this paper. We note that in a recent observational study of Cassiopeia A, DeLaney et al. (2010) deduce a flattened ejecta distribution or "thick disk" containing all the ejecta structures. They also deduce the occurrence of a roughly spherical reverse shock.

Intrinsic axial asymmetry in the form of a bipolar jet biased toward the observer has been invoked by Chugai et al. (2005) to account for the $\mathrm{H} \alpha$ profile in $\mathrm{SN} 2004 \mathrm{dj}$ up to about one year. We note that the jet makes an angle of only $\sim 15^{\circ}$ to the normal to the dust-disk plane derived from our line-profile analysis, perhaps indicating a physical connection. However, line-profile asymmetry in the earlier (pre $\sim 1$ year) nebular spectra of other CCSNe also tend to be blue-biased. This implies that, in general, an intrinsic bias toward the observer cannot be the explanation for such line blueshifts (cf. Milisavljevic et al. 2010). At later (post $\sim 1$ year) nebular epochs, line blueshifts are also often seen (Lucy et al. 1989; Spyromilio et al. 1990; Turatto et al. 1993; Fesen et al. 1999; Gerardy et al. 2000; Leonard et al. 2000; Fassia et al. 2002; Elmhamdi et al. 2003; Pozzo et al. 2004) and these are usually attributed to attenuation by newly formed dust in the ejecta or CDS. Our dust-disk model invokes this scenario. This has the advantage of explaining the red-wing suppression without invoking an intrinsic observer-biased axial asymmetry in the SN. Moreover, our model uses the same dust disk to simultaneously account for the line-profile attenuation and the MIR emission. We consider it unlikely that the Chugai et al. model can provide a superior alternative explanation for the 
line profiles presented and analyzed in Section 3.4.4.1. Further examination of the relationship between our model and that of Chugai et al. is beyond the scope of this paper.

We conclude that dust formation in the ejecta of SN 2004dj had commenced and may even have been completed, by 500 days with a near-face-on, disk-like distribution. This dust was responsible both for the late-time line-profile red-wing suppression and the bulk of the MIR luminosity up to $\sim 20 \mu \mathrm{m}$. The main source of dust heating was probably reverse-shock radiation. Assuming $\delta=0$, the dust mass was at least $10^{-4} M_{\odot}$. For $\delta=1.9$, the lower limit rises to about $3 \times 10^{-4} M_{\odot}$. The value of $\delta$ is poorly constrained. We reject silicates as the grain material.

\section{CONCLUSIONS}

We have presented optical, NIR, and MIR observations of the Type IIP SN 2004dj. The combination of wavelength and temporal coverage achieved makes this $\mathrm{SN}$ one of the most closely studied of such events. In the present work, we have analyzed the SN continuum over a period spanning 89-1393 days, augmented by a line-profile analysis over 461-925 days. Our conclusions are as follows.

1. A mass of $0.0095 \pm 0.002 M_{\odot}$ of ${ }^{56} \mathrm{Ni}$ was ejected from SN 2004dj, which is less than that reported by other authors. The period during which the radioactive tail dominated the BLC lasted for an unusually short period of only $~ 35$ days. Subsequently, a different energy source dominated; we suggest reverse-shock heating.

2. At early times, the optical/NIR ("hot") part of the continuum provided most of the SN luminosity. This emission is attributed to hot, optically thick ejecta gas. At later epochs, the optical/NIR luminosity was increasingly due to nebular emission and formed only a minor, ultimately negligible, proportion of the BLC.

3. At both early and late times, the long-wave portion of the MIR continuum (the "cold" component) was primarily due to an IS IR echo. Free-free radiation made only a minor contribution. As originally suggested by Bode \& Evans (1980), the analysis of SN IR echoes may provide a useful way of studying IS dust in nearby galaxies.

Subsequent to the submission of this paper, Szalai et al. (2011) reported their findings on SN 2004dj, making use of some of the data presented in this work. They dismiss the IS IR echo and argue against pre-existing dust since the estimated extinction of SN 2004dj was lower than that of other $\mathrm{SNe}$. We do not concur. We have shown that an extinction of only $A_{V}=0.02$ is all that is required to account for an adequate IR echo. Szalai et al. (2011) also argue that the early UV/X-ray flash would create a dust-free cavity of up to $10^{17} \mathrm{~cm}$ and that OB stars would have expelled the interstellar medium (ISM)/dust from the cluster. In fact most of the IS IR echo longward of $\sim 10 \mu \mathrm{m}$ comes from dust lying at considerably more than $1 \mathrm{pc}$. We therefore strongly favor a scenario whereby the long-wave component was primarily due to an IS IR echo.

4. The early-time NIR/MIR ("warm") component was probably due to thermal emission from non-silicate dust formed in the CDS. The CDS dust growth began at about 50 days, reached $90 \%$ of maximum by 165 days, and approached a maximum of $0.33 \times 10^{-5} M_{\odot}$; the dust mass produced in this way was small. Heating of the dust by the contemporary optical-NIR BLC completely accounts for the strength and evolution of the early-time warm component. Szalai et al. (2011) did not present contemporary optical or NIR data and consequently did not identify the early warm component.

5. The late-time warm component was dominated by the luminosity of newly formed, non-silicate dust in the ejecta. The dust growth commenced at some time between 281 days and 461 days. The same dust was responsible for the late-time red-wing attenuation of optical and NIR spectral line profiles. The dust was distributed as a nearly faceon disk, within a spherical cloud of emitting gas. During 500-996 days, the disk was effectively opaque in the optical/NIR region, but was optically thin at longer wavelengths. The dust mass appeared to grow during this period, attaining $(0.5 \pm 0.1) \times 10^{-4} M_{\odot}$ by 996 days for a uniform density disk, or a few times more than this for an $r^{-1.9}$ gradient outside $0.05 r_{\mathrm{dmax}}$ and uniform within. However, it may be that the dust mass "growth" was really due to the emergence of previously formed (i.e., pre-500 days) dust from optically thick regions of a disk with an even steeper density gradient. For the latest two epochs (1207 days and 1393 days), the dust was optically thick at all wavelengths and only lower limits could be obtained for a given gradient-for example, $>10^{-4} M_{\odot}$ for a flat gradient and a factor of three higher limit for an $r^{-1.9}$ gradient outside $0.05 r_{\text {dmax }}$ and uniform within. This is broadly in agreement with Szalai et al. (2011). For a smooth distribution, they found a dust mass of $\sim 10^{-5}-10^{-4} M_{\odot}$ though, with clumping, up to $\sim 10^{-3} M_{\odot}$ would also be possible.

6. Rather than expanding, the dust-disk radius appeared to slowly shrink. This may have been due to the dust extent being confined by the reverse shock, which also heated the grains radiatively.

7. While the latest epochs provide only lower limits to the mass of dust produced by SN 2004dj, these limits are at least a factor of 100 below the $0.1 M_{\odot}$ of grains per $\mathrm{SN}$ required to account for the dust observed at high redshifts. Moreover, measurements as late as 996 days yield actual dust masses of only $\sim 10^{-4} M_{\odot}$. While not completely ruling out the possibility that typical CCSN ejecta are major contributors to cosmic dust production, this study does suggest that, at least for SN 2004dj, the dust-mass production was small.

We thank J. Vinkó for providing us with digitized versions of his optical spectra. The work presented here is based on observations made with the Spitzer Space Telescope, the W. M. Keck Observatory, the William Herschel Telescope (WHT), and the $2.4 \mathrm{~m}$ Hiltner Telescope of the MDM Observatory. The Spitzer Space Telescope is operated by the Jet Propulsion Laboratory, California Institute of Technology, under a contract with NASA. The W. M. Keck Observatory is operated as a scientific partnership among the California Institute of Technology, the University of California, and NASA; it was made possible by the generous financial support of the W. M. Keck Foundation. We extend special gratitude to those of Hawaiian ancestry on whose sacred mountain we are privileged to be guests. The WHT is operated on the island of La Palma by the Isaac Newton Group in the Spanish Observatorio del Roque de los Muchachos of the Instituto de Astrofísica de Canarias. Financial support for this research was provided by NASA through an award issued by JPL/Caltech (specifically grant number 1322321 in the case of A.V.F.). A.V.F. gratefully acknowledges additional support from NSF grant AST-0908886 and the TABASGO 
Foundation. P.A.H. was supported by NSF grants AST-1008962 and 0708855. S.M. acknowledges support from the Academy of Finland (project 8120503). J.S. is a Royal Swedish Academy of Sciences Research Fellow supported by a grant from the Knut and Alice Wallenberg Foundation. J.C.W gratefully acknowledges support from NSF grant AST-0707769. The Dark Cosmology Centre is funded by the Danish National Research Foundation.

\section{REFERENCES}

Allen, C. W. 1973, Astrophysical Quantities (London: Athlone), 265

Andrews, J. E., et al. 2010, ApJ, 715, 541

Arnett, W. D., Bahcall, J. N., Kirshner, R. P., \& Woosley, S. E. 1989, ARA\&A, 27,629

Bendo, G. J., et al. 2010, MNRAS, 402, 1409

Bertoldi, F., Carilli, C. L., Cox, P., Fan, X., Strauss, M. A., Beelen, A., Omont, A., \& Zylka, R. 2003, A\&A, 406, L55

Beswick, R. J., Muxlow, T. W. B., Argo, M. K., Pedlar, A., Marcaide, J. M., \& Wills, K. A. 2005, ApJ, 623, 21

Bode, M. F., \& Evans, A. 1980, MNRAS, 193, 21P

Bond, H. E., et al. 2004, IAU Circ., 8385, 3

Botticella, M. T., et al. 2009, MNRAS, 398, 1041

Burrows, A., Hayes, J., \& Fryxell, B. A. 1995, ApJ, 450, 830

Cardelli, J. A., Clayton, G. C., \& Mathis, J. S. 1989, ApJ, 345, 245

Chandra, P., \& Ray, A. 2004, IAU Circ., 8397, 3

Cherchneff, I., \& Dwek, E. 2010, ApJ, 713, 1

Chevalier, R. A., \& Fransson, C. 1992, ApJ, 395, 540

Chugai, N. N. 2001, MNRAS, 326, 1448

Chugai, N. N. 2006, Astron. Lett., 32, 739

Chugai, N. N., Chevalier, R. A., \& Utrobin, V. P. 2007, ApJ, 662, 1136

Chugai, N. N., Fabrika, S. N., Sholukhova, O. N., Goranskij, V. P., Abolmasov, P. K., \& Vlasyuk, V. V. 2005, Astrophys. Lett., 31, 792

Clayton, D. D., Leising, M. D., The, L.-S., Johnson, W. N., \& Kurfess, J. D. 1992, ApJ, 399, L141

DeLaney, T., et al. 2010, ApJ, 725, 2038

Deneault, E. A.-N., Clayton, D. D., \& Meyer, B. S. 2006, ApJ, 638, 234

Di Carlo, E., et al. 2008, ApJ, 684, 471

Draine, B. T. 2003, ARA\&A, 41, 241

Draper, P. W., Gray, N., \& Berry, D. S. 2002, Starlink User Note 214.10

Dwek, E. 1983, ApJ, 274, 175

Dwek, E., \& Arendt, R. G. 2008, ApJ, 685, 976

Dwek, E., \& Cherchneff, I. 2011, ApJ, 727, 63

Dwek, E., Galliano, F., \& Jones, A. P. 2007, ApJ, 662, 927

Elmhamdi, A., et al. 2003, MNRAS, 338, 939

Ensman, L., \& Burrows, A. 1992, ApJ, 393, 742

Ercolano, B., \& Storey, P. J. 2006, MNRAS, 372, 1875

Faber, S. M., et al. 2003, Proc. SPIE, 4841, 1657

Fassia, A., Meikle, W. P. S., \& Spyromilio, J. 2002, MNRAS, 332, 296

Fassia, A., et al. 2000, MNRAS, 318, 1093

Fazio, G. G., et al. 2004, ApJS, 154, 10

Fesen, R. A., et al. 1999, AJ, 117, 725

Filippenko, A. V., Li, W., Challis, P., \& Van Dyk, S. D. 2004, IAU Circ., 8391, 2

Fischera, Jg., Tuffs, R. J., \& Völk, H. J. 2002, A\&A, 395, 189

Fransson, C., \& Kozma, C. 1993, ApJ, 408, 25

Fransson, C., et al. 2005, ApJ, 622, 991

Fraternali, F., Oosterloo, T., Sancisi, R., \& van Moorsel, G. 2001, ApJ, 562, L47 Freedman, W. L., et al. 2001, ApJ, 553, 47

Gerardy, C. L., Fesen, R. A., Höflich, P., \& Wheeler, J. C. 2000, AJ, 119, 2968

Guenther, E. W., \& Klose, S. 2004, IAU Circ., 8384, 3

Hamuy, M. 2003, ApJ, 582, 905

Houck, J. R., et al. 2004, ApJS, 154, 211

Hummer, D. G. 1988, ApJ, 327, 477

Kim, Y., Rieke, G. H., Krause, O., Misselt, K., Indebetouw, R., \& Johnson, K. E. 2008, ApJ, 678, 287

Klein, R. I., \& Chevalier, R. A. 1978, ApJ, 223, L109

Korcáková, D., et al. 2005, IBVS, 5605, 1

Kotak, R., Meikle, P., Van Dyk, S. D., Höflich, P. A., \& Mattila, S. 2005, ApJ, 628,123

Kotak, R., et al. 2009, ApJ, 704, 306

Kozma, C., \& Fransson, C. 1998, ApJ, 496, 946

Krause, O., et al. 2005, Science, 308, 1604

Leonard, D. C., Filippenko, A. V., Barth, A. J., \& Matheson, T. 2000, ApJ, 536 239
Leonard, D. C., et al. 2006, Nature, 440, 505

Li, H., McCray, R., \& Sunyaev, R. A. 1993, ApJ, 419, 824

Liu, W., \& Dalgarno, A. 1996, ApJ, 471, 480

Lucy, L. B., Danziger, I. J., Gouiffes, C., \& Bouchet, P. 1989, in Structure and Dynamics of the Interstellar Medium, ed. G. Tenorio-Tagle et al. (Berlin: Springer), 164

Maiolino, R., Schneider, R., Oliva, E., Bianchi, S., Ferrara, A., Mannucci, F., Pedani, M., \& Roca Sogorb, M. 2004, Nature, 431, 533

Maíz-Apellániz, J., Bond, H. E., Siegel, M. H., Lipkin, Y., Maoz, D., Ofek, E. O., \& Poznanski, D. 2004, ApJ, 615, L113

Mathis, J. S., Rumpl, W., \& Nordsieck, K. H. 1977, ApJ, 217, 425

Mattila, S., et al. 2008, MNRAS, 389, 141

Meikle, W. P. S., et al. 2006, ApJ, 649, 332

Meikle, W. P. S., et al. 2007, ApJ, 665, 608

Milisavljevic, D., Fesen, R. A., Gerardy, C. L., Kirshner, R. P., \& Challis, P. 2010, ApJ, 709, 1343

Misra, K., Pooley, D., Chandra, P., Bhattacharya, D., Ray, A. K., Sagar, R., \& Lewin, W. H. G. 2007, MNRAS, 381, 280

Nakano, S., Itagaki, K., Bouma, R. J., Lehky, M., \& Hornoch, K. 2004, IAU Circ., 8377,

Nozawa, T., Kozasa, T., Umeda, H., Maeda, K., \& Nomoto, K. 2003, ApJ, 598, 78

Osterbrock, D. E. 1989, Astrophysics of Gaseous Nebulae and Active Galactic Nuclei (Mill Valley, CA: Univ. Science Books)

Patat, F., Benetti, S., Pastorello, A., Filippenko, A. V., \& Aceituno, J. 2004, IAU Circ., 8378, 1

Pei, Y. C., Fall, S. M., \& Bechtold, J. 1991, ApJ, 378, 6

Pettini, M., King, D. L., Smith, L. J., \& Hunstead, R. W. 1997, ApJ, 478, 536

Pooley, D., \& Lewin, W. H. G. 2004, IAU Circ., 8390, 1

Pozzo, M., Meikle, W. P. S., Fassia, A., Geballe, T., Lundqvist, P., Chugai, N. N., \& Sollerman, J. 2004, MNRAS, 352, 457

Pun, C. S. J., et al. 1995, ApJS, 99, 223

Rieke, G., et al. 2004, ApJS, 154, 25

Rouleau, R., \& Martin, P. G. 1991, ApJ, 377, 526

Sandage, A. 1984, AJ, 89, 630

Schlegel, D. J., Finkbeiner, D. P., \& Davis, M. 1998, ApJ, 500, 525

Shortridge, K. 2002, Starlink User Note 86

Skrutskie, M. F., et al. 1997, in The Impact of Large Scale Near-IR Sky Surveys, ed. F. Garzon et al. (Astrophys. Space Sci. Lib. Vol. 210; Dordrecht: Kluwer), 25

Smith, N., Chornock, R., Silverman, J. M., Filippenko, A. V., \& Foley, R. J. 2010, ApJ, 709, 856

Smith, N., Foley, R. J., \& Filippenko, A. V. 2008, ApJ, 680, 568

Spyromilio, J., Meikle, W. P. S., \& Allen, D. A. 1990, MNRAS, 242, 669

Stockdale, C. J., Sramek, R. A., Weiler, K. W., van Dyk, S. D., Panagia, N., Pooley, D., Lewin, W., \& Marcaide, J. M. 2004, IAU Circ., 8379, 1

Szalai, T., Vinkó, J, Balog, Z, Gáspár, A, Block, M., \& Kiss, L. L. 2011, A\&A, 527,61

Timmes, F. X., Woosley, S. E., Hartmann, D. H., \& Hoffman, R. D. 1996, ApJ, 464, 332

Todini, P., \& Ferrara, A. 2001, MNRAS, 325, 726

Tominaga, N., Blinnikov, S., Baklanov, P., Morokuma, T., Nomoto, K., \& Suzuki, T. 2009, ApJ, 705, L10

Turatto, M., Cappellaro, E., Benetti, S., \& Danziger, I. J. 1993, MNRAS, 265, 471

Udalski, A., Szymanski, M., Kubiak, M., Pietrzynski, G., Soszynski, I., Wozniak, P., \& Zebrun, K. 1999, Acta Astron., 49, 201

Valiante, R., Schneider, R., Bianchi, S., \& Andersen, A. 2009, MNRAS, 397, 1661

van den Bergh, S. 1965, PASP, 77, 269

Vinkó, J., et al. 2006, MNRAS, 369, 1780

Vinkó, J., et al. 2009, ApJ, 695, 619

Wang, X., Yang, Y., Zhang, T., Ma, J., Zhou, X., Li, W., Lou, Y.-Q., \& Li, Z. 2005, ApJ, 626, 89

Weiler, K. W., Panagia, N., Montes, M. J., \& Sramek, R. A. 2002, ARA\&A, 40, 387

Werner, M. W., et al. 2004, ApJS, 154, 1

Wooden, D. H. 1997, in AIP Conf. Proc. 402, Astrophysical Implications of the Laboratory Study of Presolar Materials, ed. T. Bernatowicz \& E. Zinner (Melville, NY: AIP), 317

Wooden, D. H., Rank, D. M., Bregman, J. D., Witteborn, F. C., Tielens, A. G G. M., Cohen, M., Pinto, P. A., \& Axelrod, T. S. 1993, ApJS, 88, 477

Woosley, S. E., Pinto, P. A., \& Hartmann, D. 1989, ApJ, 346, 395

Wright, E. L. 1980, ApJ, 242, L23

Zhang, T., Wang, X., Li, W., Zhou, X., Ma, J., Jiang, Z., \& Chen, J. 2006, AJ, 131,2245

Zwitter, T., Munari, U., \& Moretti, S. 2004, IAU Circ., 8413, 1 